\title{
Guidelines
}

\section{Safety, ethical considerations, and application guidelines for the use of transcranial magnetic stimulation in clinical practice and research ${ }^{\text {th }}$}

\author{
Simone Rossi ${ }^{\mathrm{a}, *}$, Mark Hallett ${ }^{\mathrm{b}}$, Paolo M. Rossini ${ }^{\mathrm{c}, \mathrm{d}}$, Alvaro Pascual-Leone ${ }^{\mathrm{e}}$ and \\ The Safety of TMS Consensus Group ${ }^{1}$ \\ a Dipartimento di Neuroscienze, Sezione Neurologia, Università di Siena, Italy \\ ${ }^{\mathrm{b}}$ Human Motor Control Section, NINDS, NIH, Bethesda, USA \\ ${ }^{\mathrm{c}}$ Università Campus Biomedico, Roma, Italy \\ ${ }^{\mathrm{d}}$ Casa di Cura S. Raffaele, Cassino, Italy \\ ${ }^{\mathrm{e}}$ Berenson-Allen Center for Noninvasive Brain Stimulation, Beth Israel Deaconess Medical Center and Harvard Medical School, Boston, USA
}

\section{A R T I C L E I N F O}

Article history:

Accepted 21 August 2009

Available online 14 October 2009

\section{Keywords:}

Transcranial magnetic stimulation

TMS

rTMS

Safety

\begin{abstract}
A B S T R A C T
This article is based on a consensus conference, which took place in Certosa di Pontignano, Siena (Italy) on March 7-9, 2008, intended to update the previous safety guidelines for the application of transcranial magnetic stimulation (TMS) in research and clinical settings.

Over the past decade the scientific and medical community has had the opportunity to evaluate the safety record of research studies and clinical applications of TMS and repetitive TMS (rTMS). In these years the number of applications of conventional TMS has grown impressively, new paradigms of stimulation have been developed (e.g., patterned repetitive TMS) and technical advances have led to new device designs and to the real-time integration of TMS with electroencephalography (EEG), positron emission tomography (PET) and functional magnetic resonance imaging (fMRI). Thousands of healthy subjects and patients with various neurological and psychiatric diseases have undergone TMS allowing a better assessment of relative risks. The occurrence of seizures (i.e., the most serious TMS-related acute adverse effect) has been extremely rare, with most of the few new cases receiving rTMS exceeding previous guidelines, often in patients under treatment with drugs which potentially lower the seizure threshold.

The present updated guidelines review issues of risk and safety of conventional TMS protocols, address the undesired effects and risks of emerging TMS interventions, the applications of TMS in patients with
\end{abstract}

\footnotetext{
A Consensus Statement from the International Workshop on "Present and Future of TMS: Safety and Ethical Guidelines", Siena, March 7-9, 2008.

* Corresponding author. Tel.: +390577 585401; fax: +39 0577270260.

E-mail address: rossisimo@unisi.it (S. Rossi).

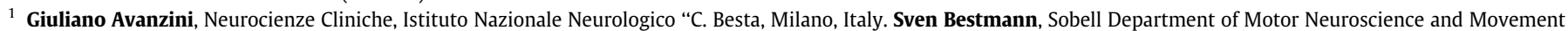

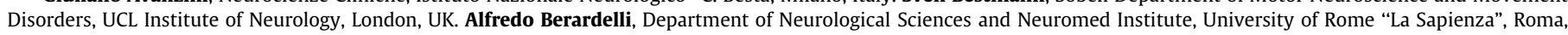

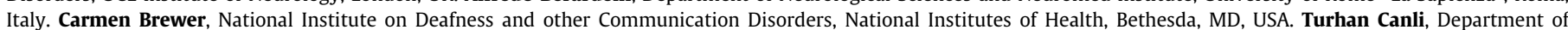

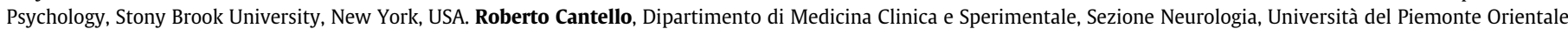

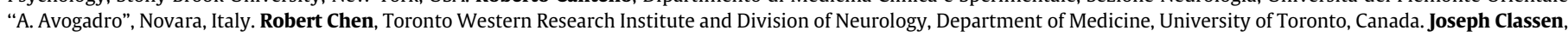

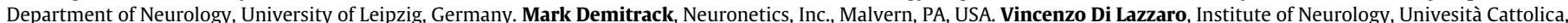

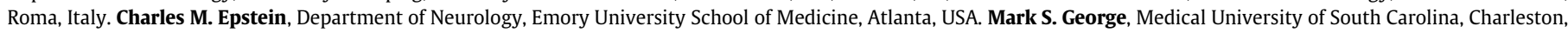

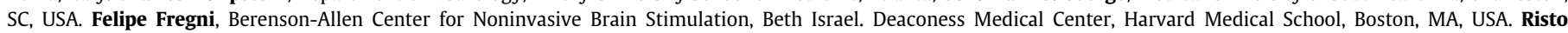

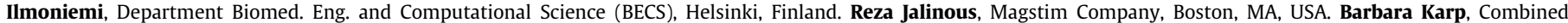

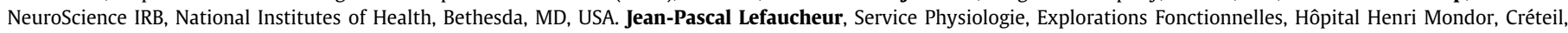

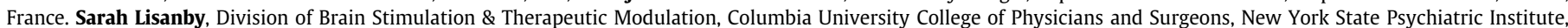

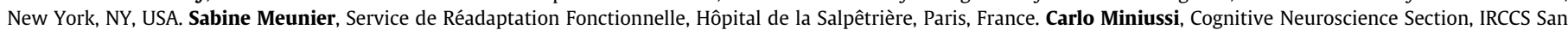

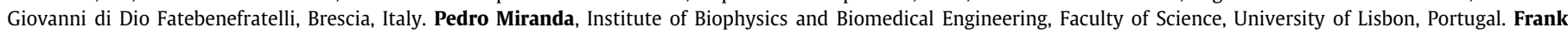

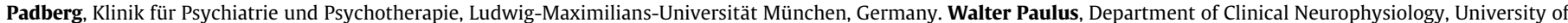

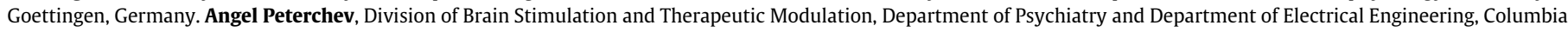

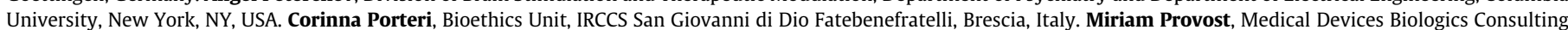

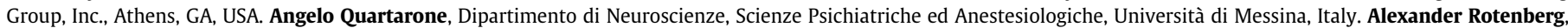

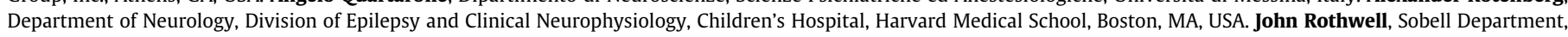

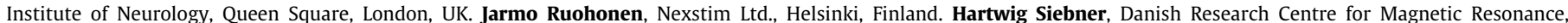

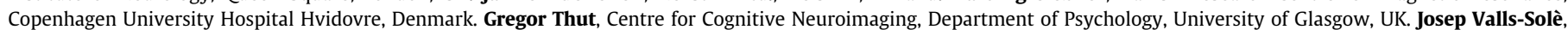

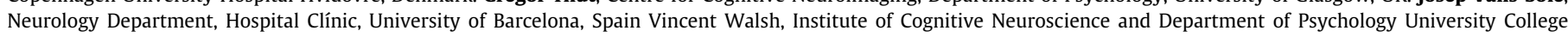

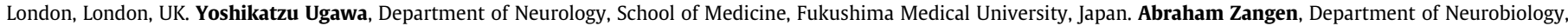
The Weizmann Institute of Science, Rehovot, Israel. Ulf Ziemann, Klinikum der Johann Wolfgang Goethe-Universität Frankfurt am Main, Germany.
} 
implanted electrodes in the central nervous system, and safety aspects of TMS in neuroimaging environments. We cover recommended limits of stimulation parameters and other important precautions, monitoring of subjects, expertise of the rTMS team, and ethical issues. While all the recommendations here are expert based, they utilize published data to the extent possible.

(c) 2009 International Federation of Clinical Neurophysiology. Published by Elsevier Ireland Ltd. All rights reserved.

\section{Contents}

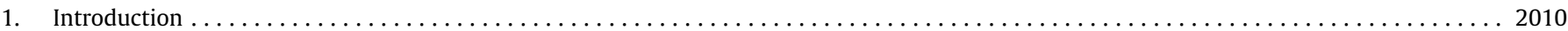

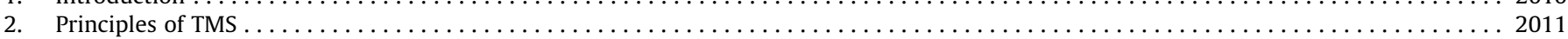

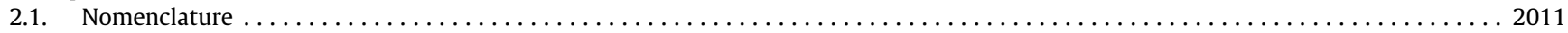

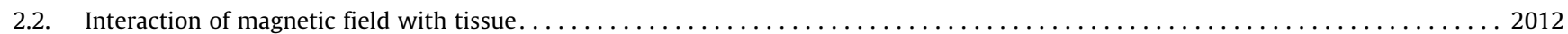

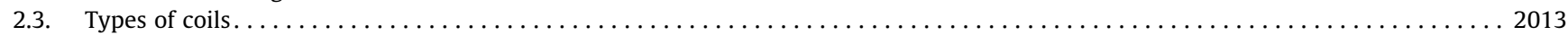

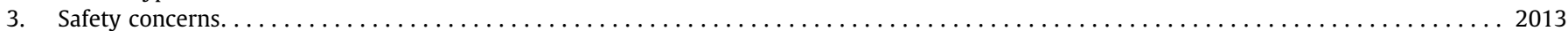

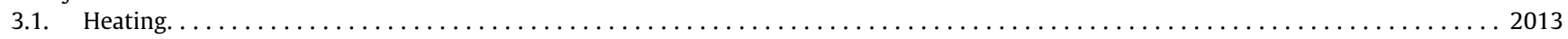

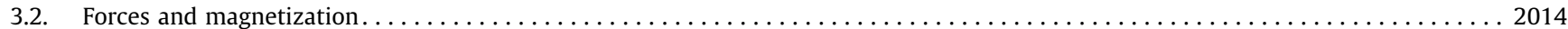

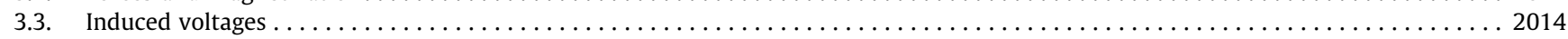

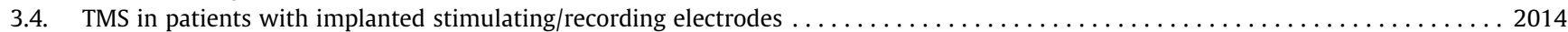

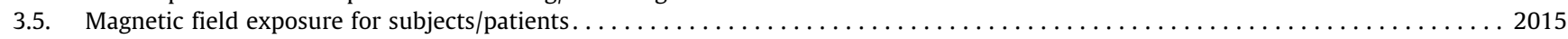

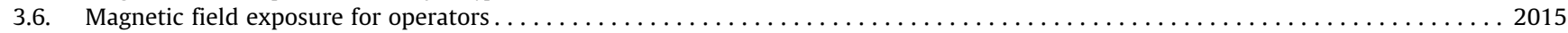

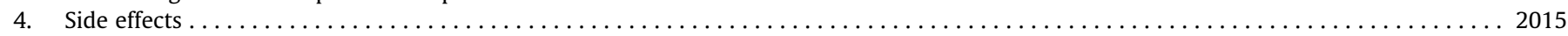

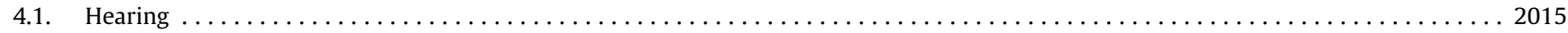

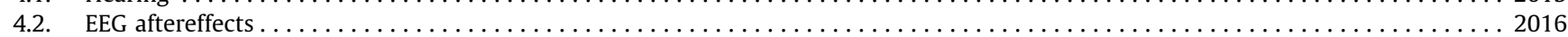

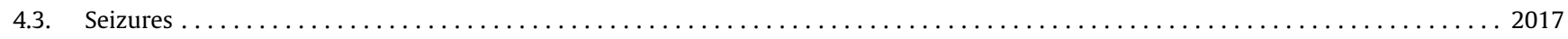

4.3.1. Seizures that have occurred with rTMS parameters considered safe according to the 1998 safety guidelines . . . . . . . . 2019

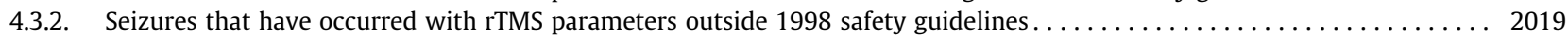

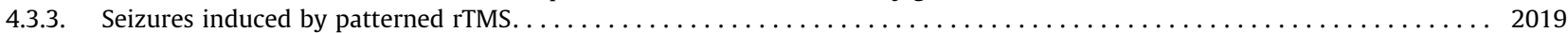

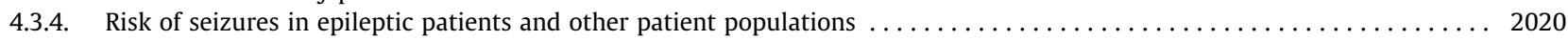

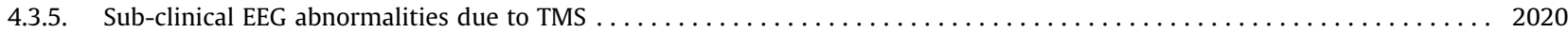

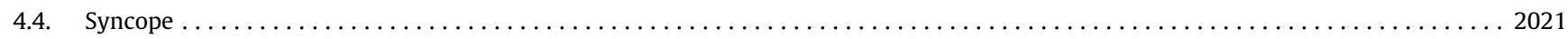

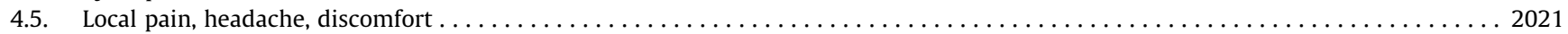

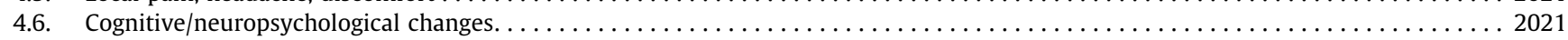

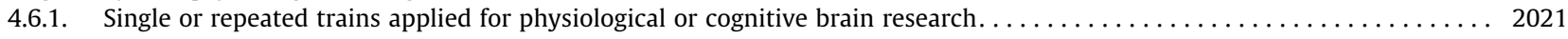

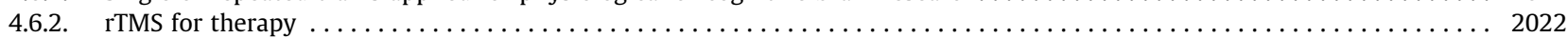

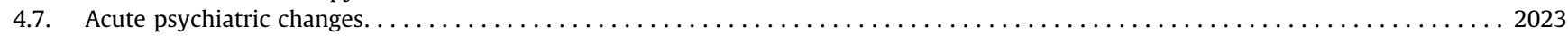

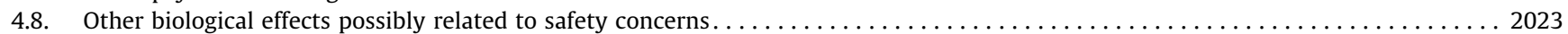

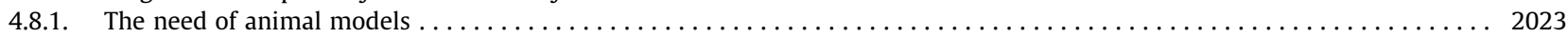

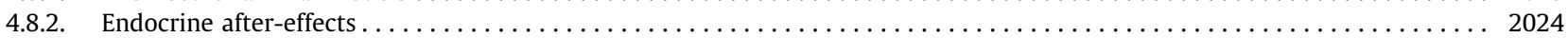

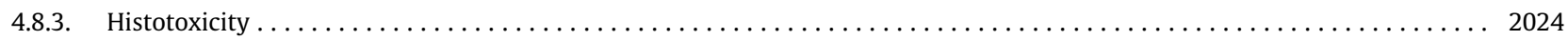

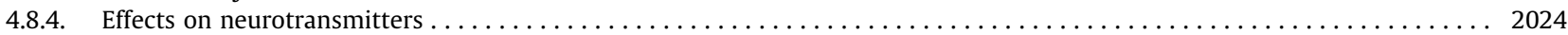

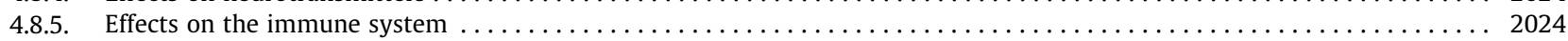

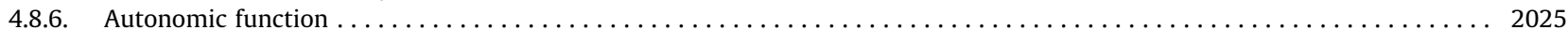

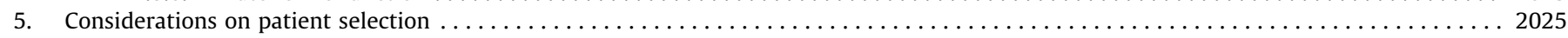

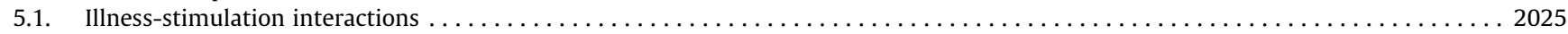

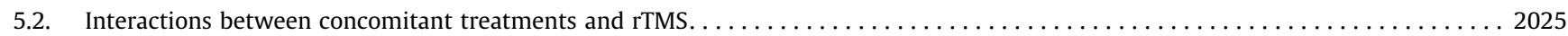

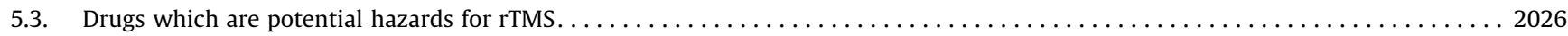

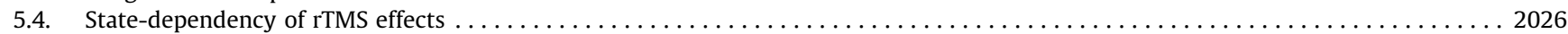

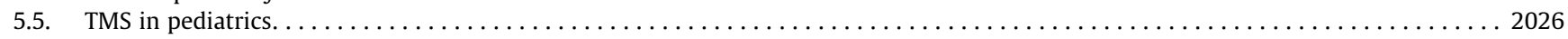

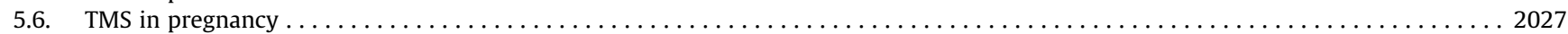

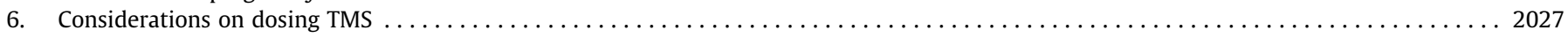

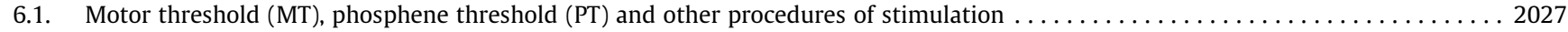

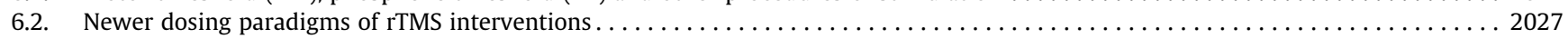

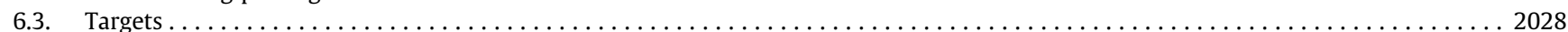

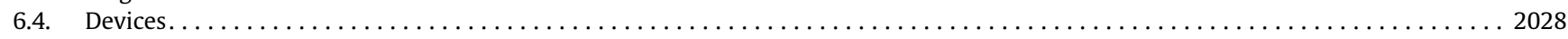

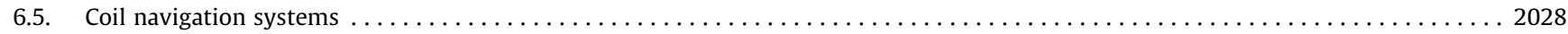

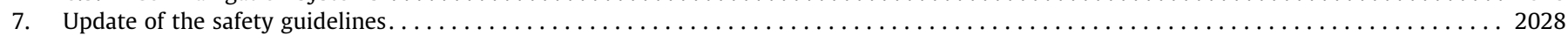

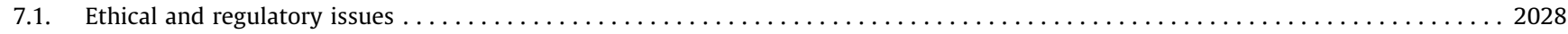

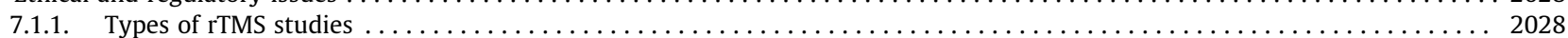

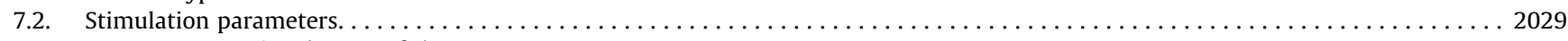

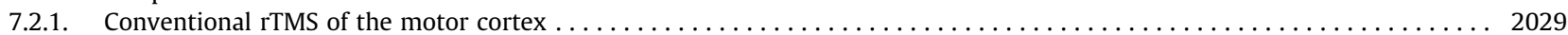

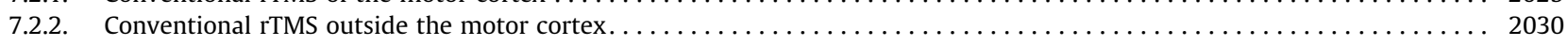

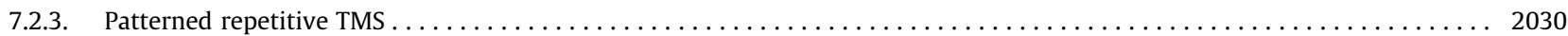

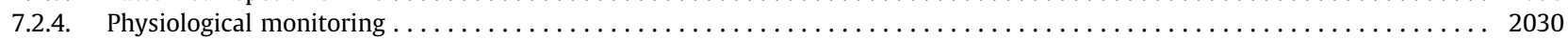

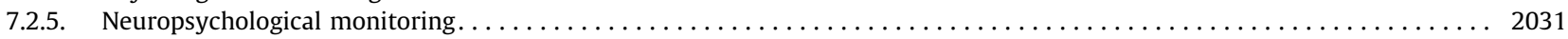

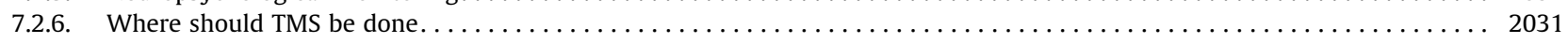

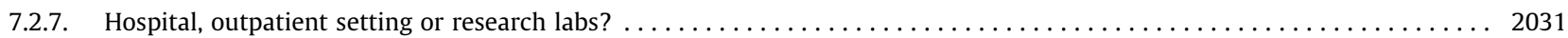

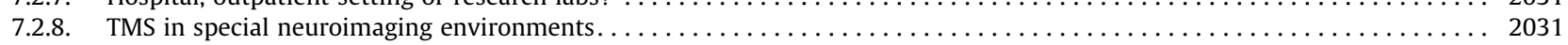

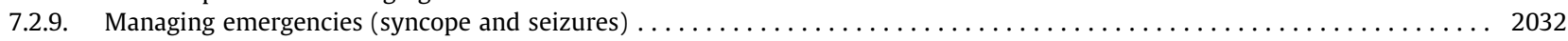




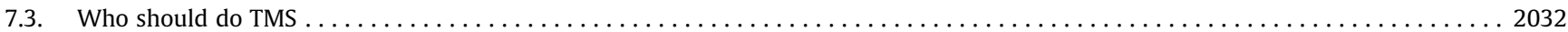

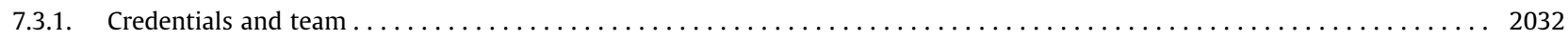

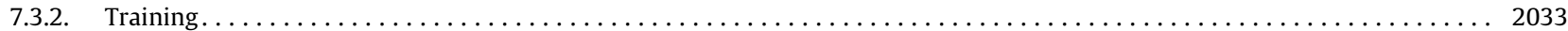

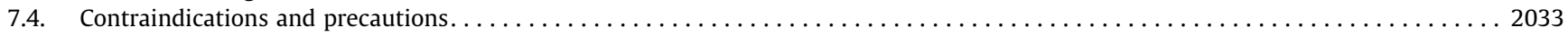

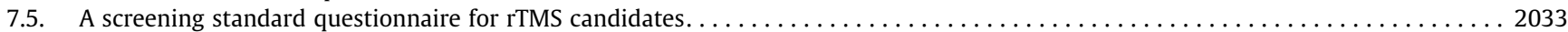

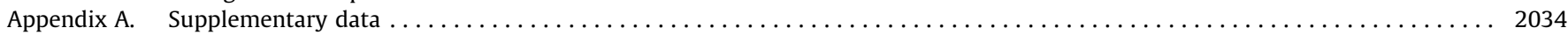

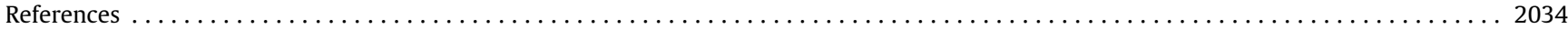

\section{Introduction}

Transcranial magnetic stimulation (TMS) is a neurostimulation and neuromodulation technique, based on the principle of electromagnetic induction of an electric field in the brain. This field can be of sufficient magnitude and density to depolarize neurons, and when TMS pulses are applied repetitively they can modulate cortical excitability, decreasing or increasing it, depending on the parameters of stimulation, even beyond the duration of the train of stimulation. This has behavioral consequences and therapeutic potential.

The last decade has seen a rapid increase in the applications of TMS to study cognition, brain-behavior relations and the pathophysiology of various neurologic and psychiatric disorders (Wassermannn and Lisanby, 2001; Kobayashi and Pascual-Leone, 2003; Gershon et al., 2003; Tassinari et al., 2003; Rossi and Rossini, 2004; Leafaucheur, 2004; Hoffman et al., 2005; Couturier, 2005; Fregni et al., 2005a,b; Hallett, 2007; George et al., 2007; Málly and Stone, 2007; Rossini and Rossi, 2007; Devlin and Watkins, 2007; Ridding and Rothwell, 2007). In addition, evidence has accumulated that demonstrates that TMS provides a valuable tool for interventional neurophysiology applications, modulating brain activity in a specific, distributed, cortico-subcortical network so as to induce controlled and controllable manipulations in behavior.

Repetitive transcranial magnetic stimulation (rTMS) has been found to be a promising noninvasive treatment for a variety of neuropsychiatric conditions (Devlin and Watkins, 2007; George et al., 2007; Aleman et al., 2007; Fregni and Pascual-Leone, 2007), and the number of applications continues to increase with a large number of ongoing clinical trials in a variety of diseases. Therapeutic utility of TMS has been claimed in the literature for psychiatric disorders, such as depression, acute mania, bipolar disorders, panic, hallucinations, obsessions/compulsions, schizophrenia, catatonia, post-traumatic stress disorder, or drug craving; neurologic diseases such as Parkinson's disease, dystonia, tics, stuttering, tinnitus, spasticity, or epilepsy; rehabilitation of aphasia or of hand function after stroke; and pain syndromes, such as neuropathic pain, visceral pain or migraine. A large industry-sponsored trial (O'Reardon et al., 2007) and a multi-center trial in Germany (Herwig et al., 2007) of rTMS in medication of refractory depression have been completed, and other appropriately controlled and sufficiently powered clinical trials of TMS are ongoing.

Most claims of therapeutic utility of TMS across conditions need further support and evidence-based clinical trial data, but the potential clinical significance is huge, affecting a large number of patients with debilitating conditions. A number of clinics have been set up worldwide offering TMS for treatment of various diseases, and rTMS is already approved by some countries for treatment of medication-refractory depression (i.e., Canada and Israel). In October 2008, a specific rTMS device was approved by the Food and Drug Administration in the United States for the treatment of patients with medication-refractory unipolar depression who have failed one good (but not more than one) pharmacological trial. It is reasonable to expect that the use of rTMS and its penetrance in the medical community will continue to increase across different medical specialties.

The number of laboratories using TMS for therapeutic or neuroscientific purposes, and consequently the number of healthy individuals and patients with various neurological or psychiatric diseases studied worldwide, has been increasing yearly for the past 20 years (Fig. 1). A further increase in the wide-spread use of TMS in medical therapeutic applications and research is expected. This makes the need for clear and updated safety guidelines and recommendations of proper practice of application critical.

Current safety precautions and practice recommendations remain guided by the consensus conference held at the National Institutes of Health in June 1996 and summarized in Clinical Neurophysiology (Wassermannn, 1998). These recommendations were adopted with minor modifications by the International Federation for Clinical Neurophysiology (Hallett et al., 1999). Ethical considerations on the application of TMS to health and disease were initially dealt with by Green et al. (1997) during the early stages of rTMS testing, and more recently have been addressed by several publications (Wolpe, 2002; Mashour et al., 2005; Illes et al., 2006; Steven and Pascual-Leone, 2006). However, as previously mentioned, the use of TMS has grown dramatically in the past decade, new protocols of TMS have been developed, changes in the devices have been implemented, TMS is being increasingly combined with other brain imaging and neurophysiologic techniques including fMRI and EEG, and a growing number of subjects and patients are being studied with expanding numbers of longer stimulation sessions.

The safety of TMS continues to be supported by recent metaanalyses of the published literature (see Machii et al., 2006; Loo et al., 2008; Janicak et al., 2008), yet there is a clear need to revisit the safety guidelines, update the recommendations of practice, and

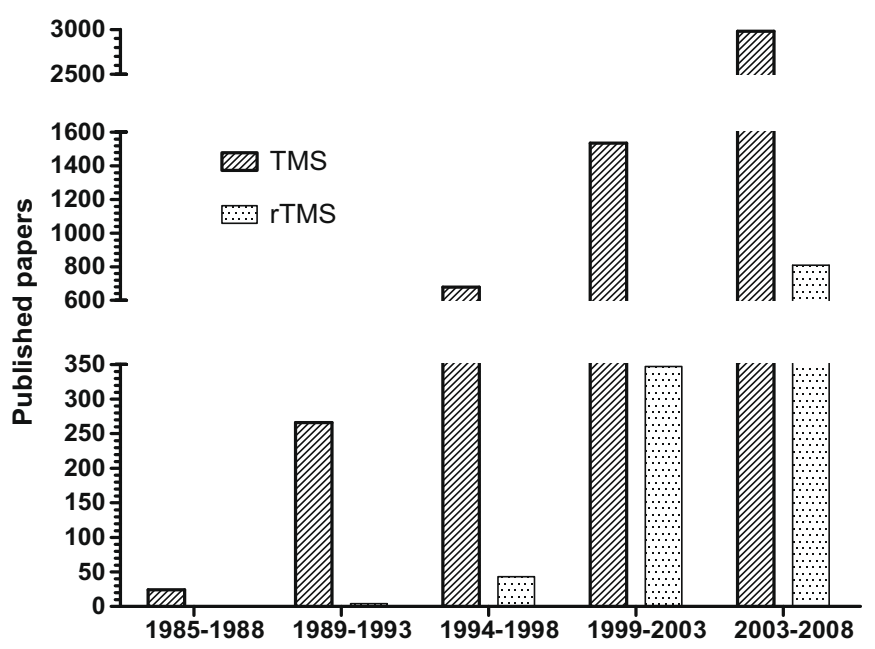

Fig. 1. Number of published papers per/year on Transcranial Magnetic Stimulation. Medline search updated to December 2008. Key words used are "Transcranial magnetic stimulation" (left bars) and "repetitive TMS" (right bars). 
improve the discussion of ethical aspect to be reflective of the expanding uses of these powerful and promising techniques. Towards this end, a consensus conference took place in Certosa di Pontignano, Siena (Italy) on March 7-9, 2008. As in the $1996 \mathrm{NIH}$ Consensus Conference, the 2008 meeting brought together some of the leading researchers in the fields of neurophysiology, neurology, cognitive neuroscience and psychiatry who are currently using TMS for research and clinical applications. In addition, representatives of all TMS equipment manufacturers were invited and those of Magstim, Nexstim, and Neuronetics were present, along with representatives from various regulatory agencies and several basic and applied scientists, including physicists, and clinicians whose work has bearing on decisions regarding the safe and ethical use of rTMS. The present article represents a summary of the issues discussed and the consensus reached. It follows the outline of the 1998 consensus statement, addressing all issues raised previously to provide corrections or updates where necessary, and including various new topics needed given technological advances.

\section{Principles of TMS}

\subsection{Nomenclature}

TMS can be applied one stimulus at a time, single-pulse TMS, in pairs of stimuli separated by a variable interval, paired-pulse TMS, or in trains, repetitive TMS. Single-pulse TMS can be used, for example, for mapping motor cortical outputs, studying central motor conduction time, and studying causal chronometry in brain-behavior relations. In paired pulse techniques TMS stimulation can be delivered to a single cortical target using the same coil or to two different brain regions using two different coils. Paired pulse

\section{Conventional rTMS}

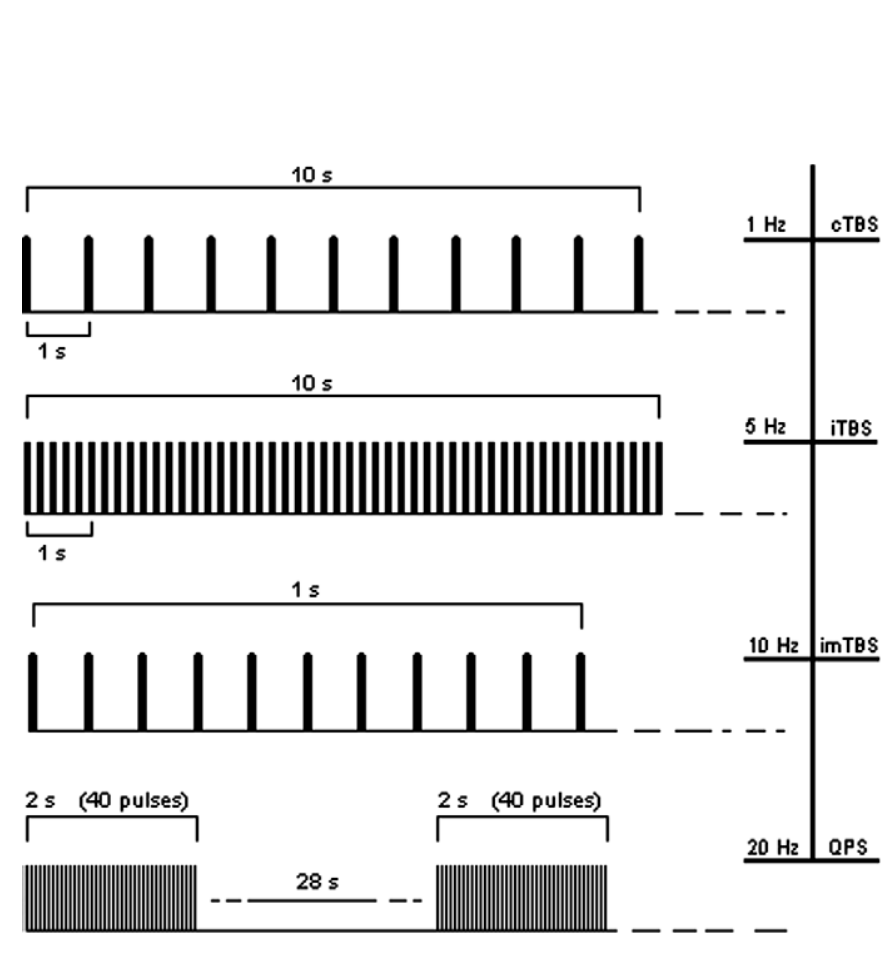

techniques can provide measures of intracortical facilitation and inhibition, as well as study cortico-cortical interactions. Pairing can also be with a peripheral stimulus and a single TMS stimulus, paired associative stimulation (PAS).

When multiple stimuli of TMS are delivered in trains, one can differentiate "conventional" and "patterned" protocols of repetitive stimulation. For conventional protocols (Fig. 2), there is universal agreement that the term 'repetitive TMS' (rTMS) has replaced earlier uses of the terms 'rapid TMS' and 'rapid-rate TMS' and should be used to refer to the application of regularly repeated single TMS pulses. The term 'fast' or 'high-frequency' rTMS should be used to refer to stimulus rates of more than $1 \mathrm{~Hz}$, and the term 'slow' or 'low-frequency' rTMS should be used to refer to stimulus rates of $1 \mathrm{~Hz}$ or less. Such a classification is based on the different physiological effects and degrees of risk associated with low- and high-frequency stimulation.

Patterned rTMS refers to repetitive application of short rTMS bursts at a high inner frequency interleaved by short pauses of no stimulation. Most used to date are the different theta burst (TBS) protocols in which short bursts of $50 \mathrm{~Hz}$ rTMS are repeated at a rate in the theta range $(5 \mathrm{~Hz})$ as a continuous (cTBS), or intermittent (iTBS) train (Huang et al., 2005; Di Lazzaro et al., 2008) (Fig. 2).

Lasting inhibitory aftereffects of $1 \mathrm{~Hz}$ rTMS and cTBS and facilitatory after-effects following high-frequency rTMS and iTBS were found on motor corticospinal output in healthy subjects, with a neurophysiologic substrate that remains unclear. Various mechanisms are worth considering, including synaptic changes resembling experimental long term depression (LTD) and long term potentiation (LTP) mechanisms, as well as shifts in network excitability, activation of feedback loops, activity-dependent
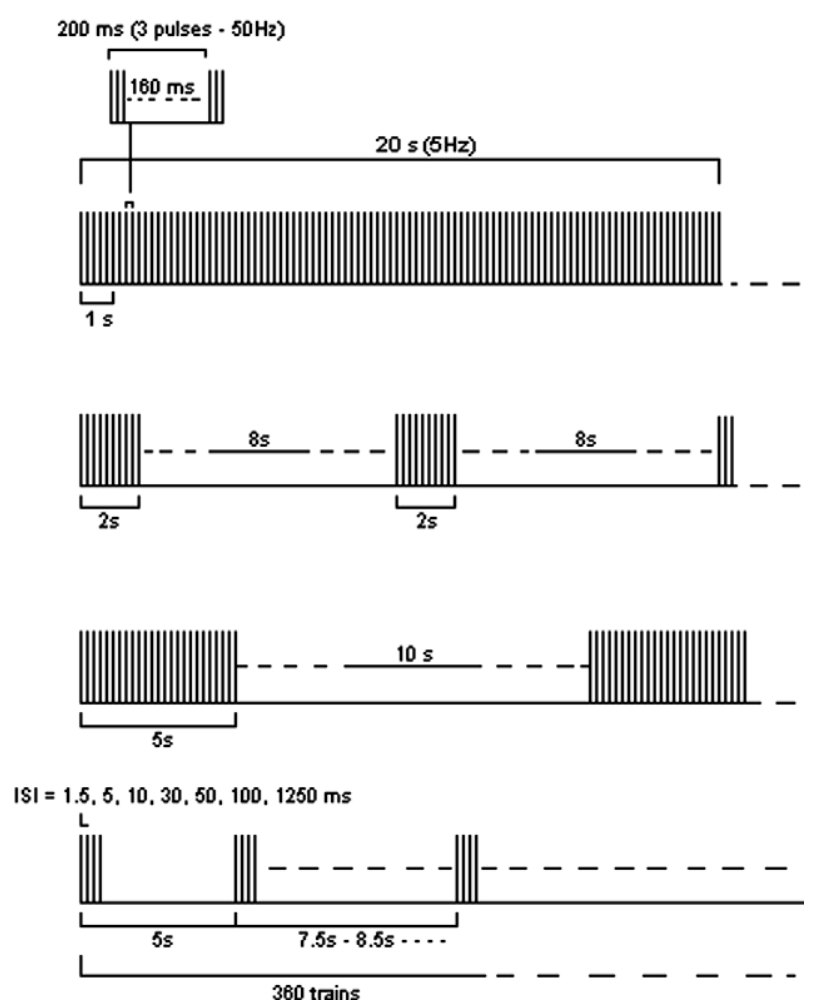

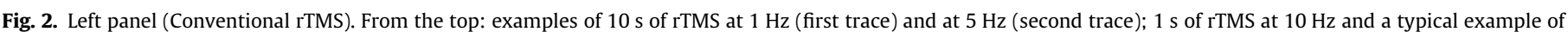

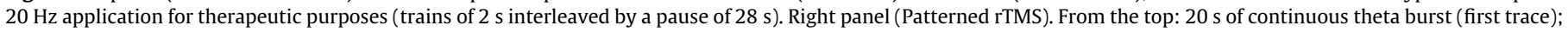
intermittent theta burst (second trace) and intermediate theta burst (third trace). The fourth trace represents protocols of quadripulse stimulations (QPS). 
metaplasticity (Gentner et al., 2008; Iezzi et al., 2008) etc. In the context of the present manuscript, a few issues are worth pointing out as they are relevant for the safety of TMS.

Regarding rhythmic, conventional repetitive, rTMS it is noteworthy, that in order to comply with present safety guidelines, protocols of slow rTMS ( $\leqslant 1 \mathrm{~Hz}$ stimulation frequency) generally apply all pulses in a continuous train, whereas protocols of fast rTMS (e.g., $\geqslant 5 \mathrm{~Hz}$ stimulation frequency) apply shorter periods of rTMS separated by periods of no stimulation (e.g., 1200 pulses at $20 \mathrm{~Hz}$ and subthreshold stimulation intensity might be delivered as 30 trains of 40 pulses ( $2 \mathrm{~s}$ duration) separated by $28 \mathrm{~s}$ intertrain intervals (Fig. 2). There is only limited safety information on the effect of inserting pauses (intertrain intervals) into rTMS protocols (Chen et al., 1997). However, considering metaplasticity arguments (Abraham and Bear, 1996; Bear, 2003), it is likely that such pauses also have a significant impact on the effect of rTMS, both in terms of efficacy and safety. Therefore, further investigations are needed.

Regarding patterned rTMS, most TBS protocols employed to date replicate the original ones explored by Huang et al. (2005): for cTBS 3 pulses at $50 \mathrm{~Hz}$ are applied at $5 \mathrm{~Hz}$ for $20 \mathrm{~s}$ (300 total stimuli) or $40 \mathrm{~s}$ ( 600 stimuli). For iTBS twenty $2 \mathrm{~s}$ periods of cTBS each separated from the following by $8 \mathrm{~s}$ are applied (Fig. 2). Obviously, there are an infinite variety of combinations of such protocols, and it is important to emphasize that the effects and safety of the different protocols may differ, and that small changes, may have profound impact.

Recently, quadripulse stimulation (QPS) (Hamada et al., 2008) has been added to patterned rTMS procedures able to induce long-term changes of cortical excitability (see Fig. 2). Repeated trains of four monophasic pulses separated by interstimulus intervals of $1.5-1250 \mathrm{~ms}$ produced facilitation (at short intervals) or inhibition (at longer intervals), probably through a modulatory action on intracortical excitatory circuitry (Hamada et al., 2008).

The combination of repeated sub-motor threshold $5 \mathrm{~Hz}$ repetitive electrical stimulation of the right median nerve synchronized with sub-motor threshold $5 \mathrm{~Hz}$ rTMS of the left M1 at a constant interval for $2 \mathrm{~min}$, or paired associated stimulation (PAS), is another protocol to temporally enhance rTMS effects at cortical level on the basis of a previously demonstrated interaction of the conditioning and test stimuli at the cortical level (Mariorenzi et al., 1991), perhaps through (meta)-plasticity mechanisms (Quartarone et al., 2006).

Repetitive paired-pulse stimulation (not included in Fig. 2) has be performed at ICF periodicity (Sommer et al., 2001) or i-wave periodicity (Di Lazzaro et al., 2007) [(also termed iTMS (Thickbroom et al., 2006) or rTMS (Hamada et al., 2007)]. Although higher excitability increases could be observed in comparison to single-pulse rTMS no seizures have been reported so far with this technique.

In all studies introducing new TMS protocols, safety should be addressed by including careful monitoring of motor, sensory and cognitive functions before, during, and after the intervention.

\subsection{Interaction of magnetic field with tissue}

In TMS, electric charge stored in a capacitor is discharged through a stimulation coil, producing a current pulse in the circuit that generates a magnetic field pulse in the vicinity of the coil. According to Faraday's law of electromagnetic induction, this time-varying magnetic field induces an electric field whose magnitude is proportional to the time rate of change of the magnetic field, which in the case of TMS is determined by the rate of change of the current in the coil. If the coil is held over a subject's head, the magnetic field penetrates scalp and skull, and induces an electric field in the brain. The induced electric field causes ions to flow in the brain, without the need for current to flow across the skull and without charged particles being injected into the scalp. In contrast, in transcranial electric stimulation (TES) charge is injected into the scalp at the electrodes and current must flow through the skull. Due to the low conductivity of the skull, in TES a large potential difference must be applied between the electrodes in order to achieve a current density in the brain high enough to stimulate neurons, and this leads to a much higher current density in the scalp. Thus, the ratio of the maximum current density in the scalp to the maximum current density in the brain is much lower in TMS than for TES, allowing TMS to stimulate cortical neurons without the pain associated with TES.

The flow of ions brought about by the electric field induced in the brain alters the electric charge stored on both sides of cell membranes, depolarizing or hyperpolarizing neurons. The existence of passive ion channels renders the membrane permeable to these ions: an increased membrane conductance decreases the amplitude of the change in membrane potential due to the induced electric field and decreases the time constant that characterizes the leakage of the induced charge. Experimental evidence (Amassian et al., 1992; Maccabee et al., 1993) and theoretical calculations (Nagarajan et al., 1993) indicate that stimulation occurs at a lower threshold where axons terminate, or bend sharply, in the relatively uniform electric field induced by the TMS stimulation coil. Accordingly, stimulation should occur where the electric field is strongest and points along the direction of an axon that terminates, for example at a synapse, or bends sharply. Axons with larger length constants, and hence larger diameters, are expected to be stimulated at lower stimulus intensity.

The stimulators and coils currently in production develop about 1.5-2.0 Tesla ( $\mathrm{T}$ ) at the face of the coil, produce currents changing at rates up to $170 \mathrm{~A} / \mu$ s (Thielscher and Kammer, 2002) and induce electric fields in the cortex of up to about $150 \mathrm{~V} / \mathrm{m}$. They are thought, depending by the stimulation intensity, to be able to activate cortical neurons at a depth of $1.5-3.0 \mathrm{~cm}$ beneath the scalp using standard Figure 8 , circular or double-cone coils. The Figure 8 coil produces a more focal and shallower stimulation, whereas the double-cone coil was especially designed for stimulation of deeper cortical targets. When using intensities below $120 \%$ of motor threshold, the stimulation can not induce direct activation at depth of more than $2 \mathrm{~cm}$ beneath the scalp (Roth et al., 2002, 2007; Zangen et al., 2005; Roth et al.,).

Stimulus waveform and current direction have a significant impact on stimulation threshold. Shorter stimulus duration requires larger pulse amplitude but lower pulse energy to achieve stimulation (Barker, 1991; Hsu et al., 2003; Peterchev et al., 2008). For monophasic pulses over the motor cortex, a lower threshold is observed when the induced current flows in the brain in posterior-anterior direction. For biphasic pulses, the threshold is lowest when the induced current flows in the posterior-anterior direction in the second phase, and hence in the opposite direction from the first phase (Kammer et al., 2001). This effect can be explained in terms of the delayed (capacitive) response of the membrane (Davey and Epstein, 2000; Corthout et al., 2001). Stimulation threshold is lower for biphasic stimuli than for monophasic stimuli only if compared in terms of the energy stored in the stimulator's capacitors. In practice, the relative value of these two thresholds may be different for different stimulators (Kammer et al., 2001), which might have relevance in terms of safety.

Several simulation models have been developed to provide a view of the electromagnetic field distributions generated in biological tissue during TMS (Wagner et al., 2007). The simplified geometries of early models argued for the absence of currents normal to the superficial cortex and limited effects of surrounding tissues or altered anatomies, but more realistic head models indicate that such conclusions are inaccurate. For example, the conjecture that radial currents are absent during TMS, has influenced the 
interpretation of clinical studies related to the generation of indirect (I) and direct (D) waves and justified the claim that inter-neurons tangential to the cortical surface are preferentially stimulated. However, such clinical interpretations need to be reevaluated in light of recent modeling work (Nadeem et al., 2003; Miranda et al., 2003; Wagner et al., 2004; De Lucia et al., 2007) that clearly demonstrate the importance of accounting for the actual head model geometry, tissue compartmentalization, tissue conductivity, permittivity, heterogeneity and anisotropy when calculating the induced electric field and current density. From a safety point of view, it is important to note that changes in the tissue anatomy and electromagnetic properties have been shown to alter the TMS induced stimulating currents in both phantom and modeling studies. Wagner et al. (2006, 2008) compared the TMS field distributions in the healthy head models with those in the presence of a stroke, atrophy or tumor. For each of these pathologies, the TMS induced currents were significantly altered for stimulation proximal to the pathological tissue alterations. The current density distributions were modified in magnitude and direction, potentially altering the population of stimulated neural elements. The main reason for this perturbation is that altered brain tissue can modify the conductivities and effectively provide paths of altered resistance along which the stimulating currents flow. Given these findings, modeling of induced electric field and current density in each patient with brain pathologies using a realistic head model, would be desirable to maximize precision. However, it is important to emphasize, that even in the absence of individualized modeling of induced currents, studies of TMS in a variety of patient populations over the past decades have proven remarkably safe if appropriate guidelines are followed.

\subsection{Types of coils}

The most commonly used coil shape in TMS studies consists of two adjacent wings, and is termed the Figure 8. This shape allows relatively focal stimulation of superficial cortical regions, underneath the central segment of the Figure 8 coil. Neuronal fibers within this region with the highest probability for being stimulated are those which are oriented parallel to the central segment of the coil (Basser and Roth, 1991; Roth and Basser, 1990; Chen et al., 2003).

The relative angle between the wings affects the efficiency and focality of the coil. Coil elements which are non-tangential to the scalp induce accumulation of surface charge, which reduces coil efficiency (Tofts, 1990; Branston and Tofts, 1991; Eaton, 1992). Hence, when the angle is smaller than $180^{\circ}$, the wings are more tangential to the scalp, and the efficiency increases (Thielscher and Kammer, 2004). Yet, a one-plane design ( $180^{\circ}$ head angle) is the most convenient form for fine localization over the head; hence it is the most commonly used.

Many studies are performed with circular coils of various sizes. Larger diameters allow direct stimulation of deeper brain regions, but are less focal. While no comparative studies have been performed to analyze the safety of circular vs. Figure 8 coils, there is no evidence for large differences in the safety parameters.

The double cone coil is formed of two large adjacent circular wings at an angle of $95^{\circ}$. This large coil induces a stronger and less focal electric field relative to a Figure 8 coil (Lontis et al., 2006), and allows direct stimulation of deeper brain regions. Because of its deep penetration, this coil allows for activation of the pelvic floor and lower limbs motor representation at the interhemispheric fissure. It is also used for cerebellar stimulation. It may induce some discomfort when higher intensities are required for stimulation of deep brain regions.

A more recent development allowing considerable reduction in power consumption and heat generation during operation, makes use of ferromagnetic cores (Epstein and Davey, 2002). The safety of such iron-core coils, using a relatively high intensity $(120 \%$ of MT) and frequency $(10 \mathrm{~Hz}, 4 \mathrm{~s}$ trains), was recently demonstrated in a large multi-center study evaluating its antidepressant effects (O’Reardon et al., 2007).

Overheating of coils during rTMS poses severe limitations on effective and safe operation, and requires an adequate cooling method. Weyh et al. (2005) introduced a Figure 8 coil with a reduced-resistance design to achieve significantly improved thermal characteristics. In addition to having increased electrical efficiency, iron-core coils offer advantages in this regard as well, as the ferromagnetic core serves as a heat sink. Water-, oil- and forced-air cooling methods have been implemented by various manufacturers.

Coil designs for stimulation of deeper brain areas, termed $\mathrm{H}$-coils, have been tested ex vivo and in human subjects (Roth et al., 2002, 2007; Zangen et al., 2005), Other theoretical designs for deep brain TMS have been evaluated with computer simulations, such as stretched C-core coil (Davey and Riehl, 2006; Deng et al., 2008) and circular crown coil (Deng et al., 2008). Coils for deep brain stimulation have larger dimensions than conventional coils, and provide a significantly slower decay rate of the electric field with distance, at the expense of reduced focality. Due to their reduced attenuation of the electric field in depth, these coils could be suitable for relatively non-focal stimulation of deeper brain structures. However, it is important to remember that as in all TMS coils, the stimulation intensity is always maximal at the surface of the brain. The safety and cognitive effects of some H-coils at relatively high intensity $(120 \% \mathrm{MT})$ and frequency $(20 \mathrm{~Hz})$ have been assessed (Levkovitz et al., 2007), and these coils have received regulatory approval for human use in Europe.

\section{Safety concerns}

\subsection{Heating}

Tissue heating of the brain by a single-pulse TMS itself is very small and is estimated to be definitely less than $0.1^{\circ} \mathrm{C}$ (Ruohonen and Ilmoniemi, 2002). It appears to be even smaller in areas with low perfusion such as cysts or strokes (R. Ilmoniemi, personal communication). However, high brain blood perfusion ensures a safety range (Brix et al., 2002). For comparison, heating in the immediate surround of deep brain stimulation electrodes is estimated to be at maximum $0.8^{\circ} \mathrm{C}$ (Elwassif et al., 2006).

Eddy currents induced in conductive surface electrodes and implants can cause them to heat up (Roth et al., 1992; Rotenberg et al., 2007). The temperature increase depends on the shape, size, orientation, conductivity, and surrounding tissue properties of the electrode or implant as well as the TMS coil type, position, and stimulation parameters. Silver and gold electrodes are highly conductive and can heat excessively, potentially causing skin burns. Temperature of $50{ }^{\circ} \mathrm{C}$ for $100 \mathrm{~s}$ or $55^{\circ} \mathrm{C}$ for $10 \mathrm{~s}$ can produce skin burns (Roth et al., 1992). The use of low-conductivity plastic electrodes can reduce heating. Radial notching of electrodes and skull plates can also reduce heating by interrupting the eddy current path. Skull plates made of titanium tend to have low heating, due to the low conductivity of titanium and radial notching (Rotenberg et al., 2007). Brain implants such as aneurysm clips and stimulation electrodes can heat as well. Brain tissue heating above $43^{\circ} \mathrm{C}$ can result in irreversible damage (Matsumi et al., 1994). If TMS is to be applied near electrodes or implants, it is advisable to first measure the heating ex vivo with the parameters specified in the planned TMS protocol. The results of such testing should be reported for the benefit of the scientific community. 


\subsection{Forces and magnetization}

The magnetic field pulse generated by the TMS coil exerts attractive forces on ferromagnetic objects and repulsive forces on non-ferromagnetic conductors. Therefore, TMS can result in forces on some head implants that could potentially displace them. The forces on ferromagnetic objects tend to be larger than those on non-ferromagnetic conductors. Titanium skull plates are non-ferromagnetic and low-conductivity, and may have radial notches which reduce the induced force. Some titanium skull plates may be safe for TMS (Rotenberg et al., 2007).

The net energy imparted to stainless steel aneurysm clips is measured to be typically less than $10^{-10} \mathrm{~J}$, equivalent to the clip being moved vertically by less than $0.0003 \mathrm{~mm}$, which is unlikely to produce a clinical problem (Barker, 1991). Cochlear implants incorporate a magnet under the scalp that could be moved or demagnetized by the TMS pulse. Analogously to the evaluation of heating, it is advisable to first measure the forces ex vivo with the parameters specified in the planned TMS protocol. Jewelry, glasses, watches and other potentially conducting or magnetic objects worn on the head should be removed during TMS to prevent interactions with the magnetic field.

\subsection{Induced voltages}

The strong magnetic field pulse emitted by the TMS coil can induce large voltages in nearby wires and electronic devices. The wires connecting to scalp electrodes should be kept free of loops and should be twisted together to reduce magnetically-induced voltages. Active brain implants, such as deep brain stimulation (DBS) systems, epidural electrode arrays for cortical stimulation, and cochlear implants contain intracranial electrodes connected to subcutaneous wires in the scalp. TMS can induce voltages in the electrode wires whether the implant is turned ON or OFF, and this can result in unintended stimulation in the brain. TMS pulses can also damage the internal circuitry of electronic implants near the coil, causing them to malfunction.

More in detail, three ex vivo studies have specifically dealt with the issue of safety (Kumar et al., 1999; Kühn et al., 2004; Schrader et al., 2005). Kumar et al. (1999) investigated the safety of TMS applied to non-implanted deep brain electrodes embedded in a conducting gel with impedance similar to the impedances found when the electrodes are in the brain. They found that the induced currents in the leads are 20 times smaller than those normally produced by the stimulator when it is used in patients, and concluded that magnetic stimulation over the coiled scalp leads does not deliver damaging stimuli to the patient's brain (Kumar et al., 1999). As a part of a study of modulation of motor cortex excitability by DBS, Kühn et al. (2004) tested the voltages induced in DBS leads in a phantom skull with methods similar to Kumar et al. (1999). They reported voltages up to $0.7 \mathrm{~V}$ induced in the electrode wires, and concluded that these are safe levels, since they are below the voltages generated by DBS. Schrader et al. (2005) assessed the effects of single-pulse TMS on a vagal nerve stimulation (VNS) device in regard to any current induced in VNS leads during TMS. They concluded that single-pulse TMS can be safely applied to individuals who have an implanted VNS device.

A significant limitation of the ex vivo safety studies (Kumar et al., 1999; Kühn et al., 2004; Schrader et al., 2005) is that only the induced voltages between pairs of contacts on the electrode lead were tested, whereas the induced voltages between the electrode contacts and the contact formed by the implanted pulse generator (IPG) case were not measured. The circuit formed by the wires connecting pairs of electrode contacts constitutes a conductive loop with a relatively small area, thus electromagnetic induction produces low voltages. On the other hand, the circuit formed by the wires connecting to the electrode contacts and the IPG case constitutes a conductive loop with a significantly larger area, and therefore electromagnetic induction can produce relatively high voltages. Thus, the induced voltages and currents reported in existing ex vivo safety studies could be significantly underestimating the magnitudes induced in vivo.

In addition to voltages and currents induced in the stimulation leads, the electromagnetic pulse generated by TMS can cause malfunction or even damage in the internal circuitry of electronic implants near the TMS coil. TMS pulses delivered ex vivo at a distance of $2-10 \mathrm{~cm}$ from the TMS coil to DBS IPG caused the IPG to malfunction, and for distances of less than $2 \mathrm{~cm}$, the IPG was permanently damaged (Kumar et al., 1999; Kühn et al., 2004). A similar study of the effect of TMS pulses on a VNS IPG did not detect signs of malfunction or damage to the IPG by the TMS pulse (Schrader et al., 2005).

Cochlear implants consist of a loop antenna, a permanent magnet, an electronic chip implanted under the scalp, and an electrode implanted in the cochlea. There is no safety data on TMS in subjects with cochlear implants, but basic physics considerations suggest that it is likely unsafe. The TMS pulse can induce high voltages in the loop antenna, can move or demagnetize the permanent magnet, and can cause malfunction or damage to the electronic chip. Further, cochlear implants are not MRI compatible. Therefore, TMS should not be performed in subjects with cochlear implants, unless a detailed safety evaluation proves there are no adverse effects.

\subsection{TMS in patients with implanted stimulating/recording electrodes}

A large number of TMS studies have been performed in patients with electrodes implanted both in central and peripheral nervous system. Most employed single-pulse TMS, some used paired pulse TMS and a few studies used repetitive TMS (see Supplemental material, Table S1). The main aims of such studies have been:

(a) Evaluation of the effects of TMS on the central nervous system activity either by recording the responses evoked by TMS or by evaluating the changes of the ongoing spontaneous electrophysiological activity after TMS through the implanted electrodes;

(b) Evaluation of the effects of stimulation of nervous system structures by the implanted electrodes, as revealed by TMS evoked responses.

The first in vivo study with spinal cord stimulators was performed by Kofler et al. (1991) in four patients, and they reported that TMS was safely applied with the devices turned OFF and ON, with no apparent adverse effect (Kofler et al., 1991). Since then, studies performed in patients with implanted electrodes (see Supplemental material, Table S1) have used mainly three types of electrodes: (1) epidural electrodes (implanted over the cerebral cortex or spinal cord); (2) deep brain electrodes; or (3) peripheral or cranial nerve stimulating electrodes (e.g., vagus nerve (VN) electrodes). Some of the studies were performed in the few days following implantation, whilst the electrode leads were externalized before connection to a subcutaneous stimulus generator, while other studies were performed in patients with the leads connected to implanted stimulators. Two of the latter studies (Kühn et al., 2002; Hidding et al. 2006) showed that TMS-induced lead currents can produce motor responses in vivo, suggesting that the magnitude of these currents was higher than the negligible levels measured ex vivo. This phenomenon could be explained by currents induced between the electrode contacts and the IPG case, which were not measured in the ex vivo tests (see Section 3.3). Kühn et al. (2002) performed TMS in 5 dystonic patients with implanted electrodes in globus pallidus 
internus. These authors suggested that TMS can induce currents in the subcutaneous wire loops in patients with implanted DBS electrodes which are sufficient to activate corticospinal fibres subcortically and to elicit pseudo-ipsilateral hand motor responses (Kühn et al., 2002). Similar findings were reported in 8 parkinsonian patients with subthalamic nucleus (STN) electrodes and leads connected to an implanted stimulator (Hidding et al., 2006). The mean onset latencies of motor responses recorded in the relaxed first dorsal interosseous muscle were significantly shorter after electrode implantation compared to the preoperative state. The authors ascribed the shortening of the corticomotor conduction time to inadvertent stimulation of fast-conducting descending neural elements in the vicinity of the STN through current induction in subcutaneous scalp leads underneath the TMS coil connecting the external stimulator with STN electrodes, thereby producing submotor threshold descending volleys. Importantly though, no adverse effects were reported by Kühn et al. (2002) and by Hidding et al. (2006).

In summary, based on ex vivo and in vivo studies, it appears that TMS can be safely applied to patients who have implanted stimulators of the central and peripheral nervous system when the TMS coil is not in close proximity to the internal pulse generator (IPG) system. However, we lack detailed information as to what constitutes a safe distance between the TMS coil and the implanted stimulator, and how coil shape, coil angulation, etc. influence this relation. Therefore, TMS should only be done in patients with implanted stimulators if there are scientifically or medically compelling reasons justifying it. TMS procedures need to strictly follow a pre-specified experimental protocol and setting, with appropriate oversight by the Institutional Review Board or Ethic Committee. In such instances, to prevent accidental firing of the TMS coil near electronic implants, the subjects could wear a lifejacket or a similar arrangement which provides about $10 \mathrm{~cm}$ of padding around the electronic implant (Schrader et al., 2005).

TMS is considered safe in individuals with VNS systems (Schrader et al., 2005), cardiac pacemakers, and spinal cord stimulators as long as the TMS coil is not activated near the components located in the neck or chest. If a TMS coil is discharged close to the implanted wires connecting the electrodes to the IPG, potentially significant voltages and currents could be induced between the electrode leads and the IPG, which could cause unintended neural stimulation and may present a safety risk. This scenario can occur in DBS and cortical stimulation with epidural electrodes. Additional safety studies should be conducted to evaluate the magnitude of the voltages and currents induced in implanted stimulation systems. Finally, TMS in subjects with cochlear implants should not be performed, due to multiple possibly unsafe interactions between the TMS pulse and the implant.

\subsection{Magnetic field exposure for subjects/patients}

Single sessions of TMS or rTMS do not carry the risk of significant magnetic field exposure since the total time is too short. However, a typical treatment course of rTMS for a psychiatric application (e.g., $10 \mathrm{~Hz}$, trains of 20 pulses, $5 \times$ s, 20 sessions) yields about $5 \mathrm{~s}$ of total exposure (Loo et al., 2008). Theoretically, this kind of exposure would fall into radiofrequency range (i.e., from $3 \mathrm{kHz}$ to $300 \mathrm{GHz}$ ), assuming a continuous stimulation with each pulse lasting about $250 \mu$ s (Barker, 1991).

In a current TMS depression trial, the researchers (M. George, personal communication) are delivering 6000 stimuli in a day ( $120 \%$ of MT, $10 \mathrm{~Hz}, 5 \mathrm{~s}$ on-10 off, for $30 \mathrm{~min}$ each day), in an open-ended dynamically adaptive design where they treat to remission as long as there is continued improvement. There is a maintenance phase and patients can be retreated if they relapse. One 28-year old patient has now received 70 sessions over 12 months, or 420.000 pulses, with no side effects. Several patients with amyotrophic lateral sclerosis have also received a very prolonged treatment using cTBS. One 75-year old patient has received 130 sessions over 26 months with a total number of 156,000 stimuli, while 7 patients received 60 sessions over 12 months with a total number of 72,000 stimuli (Di Lazzaro et al., 2009).

As pointed out (Loo et al., 2008), it is unclear whether the high intensity, pulsed stimulation of TMS has the same long-term effects of continuous, low-intensity, occupational exposure. It is even less clear whether effects of long-term exposure to rTMS might be changed by concurrent medications. Prospective studies in this sense would be desirable. Nonetheless, it is worth noting that chronic exposure to electro-magnetic fields appears safe at levels even greater than those possible with TMS (Gandhi, 2002; Martens, 2007).

\subsection{Magnetic field exposure for operators}

Safety issues are rarely addressed for operators who are exposed to magnetic field several hours every day for years by performing TMS. Guidelines for occupational levels of exposure to electromagnetic fields have been proposed by the International Commission on Non-Ionizing Radiation Protection (see ICNIRP, 2003) and by a Directive from the European Parliament [directive 2004/40/EC (Riches et al., 2007a)]. This directive introduces Exposure Limit Values for workers and also Action Values (magnitude of electromagnetic field which is directly measurable). In contrast, long term effects have been excluded from the scope of the directive. This directive has been operational from 30 April 2008 in all countries of the European Union (now postponed to April 30, 2012). Occupational exposure to magnetic fields has been measured for MRI units (Riches et al., 2007a). Exposure values are 100 times below the recommended exposure limits (Bradley et al., 2007), except in case of interventional procedures (Hill et al., 2005; Riches et al., 2007b).

Regarding TMS/rTMS, only one study has been performed using the MagPro machine (Medtronic), MC-B70 Figure 8 coil, $5 \mathrm{~Hz}$ frequency, and stimulus intensity of $60-80 \%$ stimulator output (Karlström et al., 2006). In these conditions, worker's exposure limits for the magnetic field pulses are transgressed at a distances of about $0.7 \mathrm{~m}$ from the surface of the coil. This single observation makes necessary further research to confirm it and to determine the limiting distance to the coil according to the type of TMS machine, the type of coil, the frequency/intensity of stimulation and the total exposure time.

The potential risk of long-term adverse event for rTMS operators due to daily close exposure (even to weak electromagnetic fields), repeated for years, is an open issue that should be addressed in the future.

\section{Side effects}

All the known side effects linked with TMS use are summarized in Table 1. It is apparent that data on theta burst stimulation (TBS) are still not sufficient to claim or deny safety hazards. This implies that future therapeutic and research studies employing TBS and other forms of patterned repetitive TMS should explicitly address this issue, which has been neglected up to now. Below, the most significant, potential side effects of conventional TMS are commented on in further detail, including potentially hazardous TMS-related activity (see points 3.1-3.8):

\subsection{Hearing}

Rapid mechanical deformation of the TMS stimulating coil when it is energized produces an intense, broadband acoustic artifact that may exceed $140 \mathrm{~dB}$ of sound pressure level (Counter and 
Table 1

Potential side effects of TMS. Consensus has been reached for this table.

\begin{tabular}{|c|c|c|c|c|c|}
\hline Side effect & $\begin{array}{l}\text { Single-pulse } \\
\text { TMS }\end{array}$ & Paired-pulse TMS & Low frequency rTMS & High frequency rTMS & Theta burst \\
\hline Seizure induction & Rare & Not reported & $\begin{array}{l}\text { Rare (usually } \\
\text { protective effect) }\end{array}$ & $\begin{array}{l}\text { Possible ( } 1.4 \% \text { crude risk estimate } \\
\text { in epileptic patients; less than } \\
1 \% \text { in normals) }\end{array}$ & $\begin{array}{l}\text { Possible (one seizure in a } \\
\text { normal subject during } \\
\text { cTBS) } \\
\text { (see para } 3.3 .3 \text { ) }\end{array}$ \\
\hline $\begin{array}{l}\text { Transient acute } \\
\text { hypomania induction }\end{array}$ & No & No & Rare & $\begin{array}{l}\text { Possible following left prefrontal } \\
\text { stimulation }\end{array}$ & Not reported \\
\hline Syncope & \multicolumn{4}{|c|}{ Possible as epiphenomenon (i.e., not related to direct brain effect) } & Possible \\
\hline $\begin{array}{l}\text { Transient headache, local pain, } \\
\text { neck pain, toothache, } \\
\text { paresthesia }\end{array}$ & Possible & $\begin{array}{l}\text { Likely possible, but } \\
\text { not reported/ } \\
\text { addressed }\end{array}$ & $\begin{array}{l}\text { Frequent (see para. } \\
3.3 \text { ) }\end{array}$ & Frequent (see para. 3.3) & Possible \\
\hline Transient hearing changes & Possible & $\begin{array}{l}\text { Likely possible, but } \\
\text { not reported }\end{array}$ & Possible & Possible & Not reported \\
\hline $\begin{array}{l}\text { Transient cognitive/ } \\
\text { neuropsychologial changes }\end{array}$ & Not reported & No reported & $\begin{array}{l}\text { Overall negligible } \\
\text { (see Section } 4.6 \text { ) }\end{array}$ & Overall negligible (see Section 4.6) & $\begin{array}{l}\text { Transient impairment of } \\
\text { working memory }\end{array}$ \\
\hline Burns from scalp electrodes & No & No & Not reported & Occasionally reported & $\begin{array}{l}\text { Not reported, but } \\
\text { likely possible }\end{array}$ \\
\hline $\begin{array}{l}\text { Induced currents in } \\
\text { electrical circuits }\end{array}$ & \multicolumn{5}{|c|}{$\begin{array}{l}\text { Theoretically possible, but described malfunction only if TMS is delivered in close proximity with the electric device (pace-makers, } \\
\text { brain stimulators, pumps, intracardiac lines, cochlear implants) }\end{array}$} \\
\hline Structural brain changes & Not reported & Nor reported & Inconsistent & Inconsistent & Not reported \\
\hline Histotoxicity & No & No & Inconsistent & Inconsistent & Not reported \\
\hline $\begin{array}{l}\text { Other biological } \\
\text { transient effects }\end{array}$ & Not reported & Not reported & Not reported & $\begin{array}{l}\text { Transient hormone (TSH), } \\
\text { and blood lactate } \\
\text { levels changes }\end{array}$ & Not reported \\
\hline
\end{tabular}

Borg, 1992). This exceeds the recommended safety levels for the auditory system (OSHA). Before using a given coil/stimulator, the operator may consult the manufacturer's Instructions for use or technical specifications to check the specified sound pressure levels.

After exposure to the TMS stimulus, a small proportion of adult humans have experienced transient increases in auditory thresholds (Pascual-Leone et al., 1992; Loo et al., 2001). Permanent threshold shift has been observed in a single individual who did not have ear plugs and was being stimulated with an $\mathrm{H}$-coil (Zangen et al., 2005). The majority of studies in which hearing protection was used report no change in hearing after TMS (Pascual-Leone et al., 1991; Levkovitz et al., 2007; Folmer et al., 2006; Rossi et al., 2007a; Janicak et al., 2008). The single publication regarding hearing safety in pediatric cases reports no change in hearing in a group of 18 children without hearing protection (Collado-Corona et al., 2001). This is encouraging; however, the sample size is too small to ensure hearing safety for pediatric cases. Young children are of particular concern because their canal resonance is different from adults, their smaller head size results in the TMS coil being closer to the ear, and appropriate hearing protection devices for children are not available.

Therefore, it is recommended that:

(1) Hearing safety concerns for adults be addressed by: (i) use of approved hearing protection (earplugs or ear muffs) by individuals trained in placement of these devices; (ii) prompt referral for auditory assessment of all individuals who complain of hearing loss, tinnitus, or aural fullness following completion of TMS; (iii) those with known pre-existing noise induced hearing loss or concurrent treatment with ototoxic medications (Aminoglycosides, Cisplatine) should receive TMS only in cases of a favorable risk/benefit ratio, as when rTMS is used for treatment of tinnitus.

(2) Individuals with cochlear implants should not receive TMS (see also paragraphs 2.2 and 2.3).
(3) The acoustic output of newly developed coils should be evaluated and hearing safety studies should be conducted as indicated by these measures.

(4) Hearing safety concerns for children have not been sufficiently addressed in published literature (see also paragraph 4.5 ) to justify participation by pediatric healthy volunteers in TMS studies until more safety data are available. Application of rTMS in pediatric patient populations with therapeutic intent may be reasonable if the potential benefits outweigh the theoretical risks of hearing problems.

\subsection{EEG aftereffects}

Recording of electroencephalographic (EEG) activity immediately before, during, and after TMS is possible provided that certain technical challenges are addressed and few precautions taken (Ilmoniemi et al., 1997; Bonato et al., 2006; Thut et al., 2005; Ives et al., 2006; Morbidi et al., 2007). Problems related to the saturation of the EEG recording amplifiers from the TMS pulse have been overcome via artifact subtraction, pin-and-hold circuits, the use of modified electrodes which do not transiently change their shape due to the stimulus impact, and altering the slew rate of the preamplifiers.

There is a considerable number of publications of combined TMSEEG to date ( 85 studies on more than 1000 volunteers over the last 19 years). The studies that quantified aftereffects on EEG activity induced by conventional or patterned rTMS are listed in Table S2 (supplemantal material) and discussed in this section. The studies on EEG-aftereffects in the form of potential TMS-induced epileptiform EEG-abnormalities are listed in Table 2 and discussed in Section 4.3.5. Single-pulse studies are not included in either table since safety concerns did not arise. However, in Table 2, special emphasis is placed on patient populations who might be more vulnerable to TMS due to several factors (i.e., brain damage, drug treatment or discontinuation of treatment for the purpose of a study). 
Table 2

Inspection of EEG for epileptiform abnormalities during or after repetitive TMS in patients and healthy subjects. Consensus has been reached for this table.

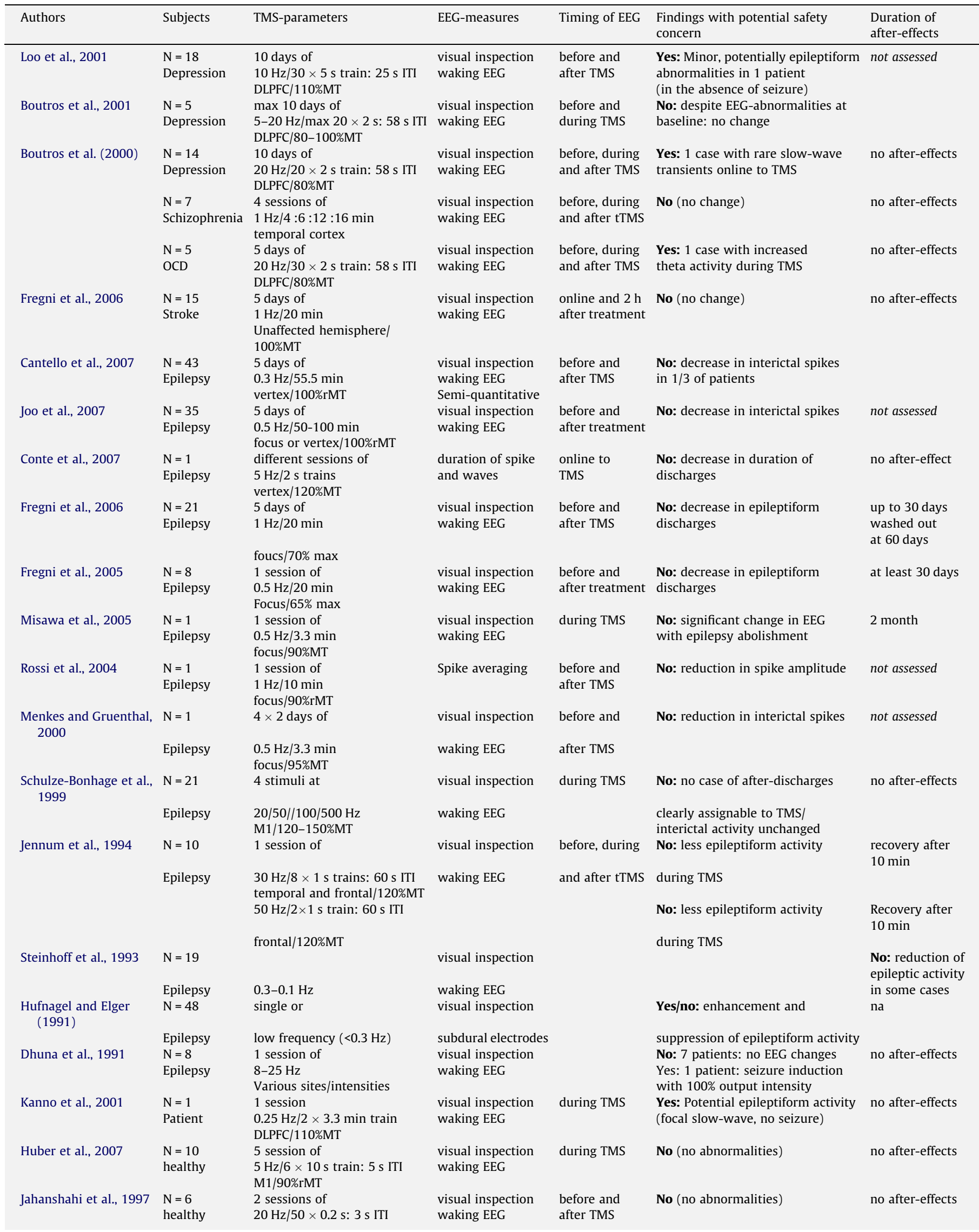


Table 2 (continued)

\begin{tabular}{|c|c|c|c|c|c|c|}
\hline Authors & Subjects & TMS-parameters & EEG-measures & Timing of EEG & $\begin{array}{l}\text { Findings with potential safety } \\
\text { concern }\end{array}$ & $\begin{array}{l}\text { Duration of } \\
\text { after-effects }\end{array}$ \\
\hline \multirow[t]{2}{*}{$\begin{array}{l}\text { Wassermannn et al., } \\
1996\end{array}$} & $\begin{array}{l}\mathrm{N}=10 \\
\text { healthy }\end{array}$ & $\begin{array}{l}\text { M1/105- } 110 \% \mathrm{aMT} \\
1 \mathrm{session} \text { of } \\
1 \mathrm{~Hz} / \mathrm{max} 5 \mathrm{~min} \\
6 \text { scalp positions } / 125 \% \mathrm{rMT}\end{array}$ & $\begin{array}{l}\text { visual inspection } \\
\text { waking EEG }\end{array}$ & $\begin{array}{l}\text { before and } \\
\text { after TMS }\end{array}$ & No (no abnormalities) & no after-effects \\
\hline & $\begin{array}{l}\mathrm{N}=10 \\
\text { healthy }\end{array}$ & $\begin{array}{l}1 \text { session of } \\
20 \mathrm{~Hz} / 10 \times 2 \text { s train: } 58 \mathrm{~s} \text { ITI }\end{array}$ & $\begin{array}{l}\text { visual inspection } \\
\text { waking EEG }\end{array}$ & $\begin{array}{l}\text { before and } \\
\text { after TMS }\end{array}$ & No (no abnormalities) & no after-effects \\
\hline
\end{tabular}

Thirty-seven studies have quantified the aftereffects on functional EEG-activity due to TMS pulse repetition (Supplemental material, Table S2). Published from 1998 to 2008, most of these studies used conventional rTMS protocols (pulse repetition frequencies of 0.9-25 Hz) which followed the 1998-safety guidelines. A few studies explored EEG aftereffects after TBS (Katayama and Rothwell, 2007, Ishikawa et al., 2007), or PAS (Tsuji and Rothwell, 2002; Wolters et al., 2005). Aftereffects have been observed on a variety of EEG/EP-measures including oscillatory activity over motor and prefrontal areas (e.g., Strens et al., 2002 and Schutter et al., 2003) as well as somatosensory (e.g., Katayama and Rothwell, 2007; Ishikawa et al., 2007; Restuccia et al., 2007), visual (Schutter et al., 2003), cognitive (Evers et al., 2001a, b; Hansenne et al., 2004; Jing et al., 2001) and movement-related cortical potentials (Rossi et al., 2000; Lu et al., 2009), in accordance with the site of TMS. With three exceptions (Evers et al., 2001a, 2001b; Satow et al., 2003; Hansenne et al., 2004), all published studies reported significant aftereffects. For the studies using conventional rTMS protocols (low frequency: 0.9 and $1 \mathrm{~Hz}$, high frequency: $5-25 \mathrm{~Hz}$ ), the direction of the aftereffect (when present) was as expected, with facilitation prevailing over suppression after high frequency TMS ( $n=12$ vs. $n=6$ ), and suppression prevailing over facilitation after low frequency TMS $(n=13$ vs. $n=2)$. Note that alpha-band increase/decrease is taken as sign for inhibition/ facilitation. TBS and PAS tended to induce facilitation rather than inhibition (small $n$ of 4, see Supplemental material, Table S2).

There is evidence for such aftereffects to persist in the absence of behavioral effects (e.g., Rossi et al., 2000; Hansenne et al., 2004; Holler et al., 2006). This parallels previous findings on lasting changes in neurophysiological measures after rTMS over motor areas (MEP-amplitude) without parallel changes in amplitude or velocity of voluntary finger movements (e.g., Muellbacher et al., 2000).

Absolute duration of EEG aftereffects has been assessed in a total of 11 studies recording EEG/EPs until recovery. Duration ranged from $20 \mathrm{~min}$ (Tsuji and Rothwell, 2002; Thut et al., 2003a, 2003b) to $70 \mathrm{~min}$ (Enomoto et al., 2001) post-TMS (mean: $38.6 \mathrm{~min}$; see Supplemental material, Table S2). There was no consistency in one type of protocol inducing longer-lasting effects than another ( $1 \mathrm{~Hz}=38 \mathrm{~min}$ : mean of $n=5 ; 5 \mathrm{~Hz}=28 \mathrm{~min}$ : mean of $n=2$; TBS $=60$ min: $n=1$; PAS $=40$ : mean of $n=2$ ), but this is preliminary given the small number of studies and the cross-study confounds of variations in number of pulses and intensity. The 20-70 min duration is in line with the duration of aftereffects on motor cortex excitability as measured through a variety of single and paired-pulse TMS protocols (e.g., Gerschlager et al., 2001; Münchau et al., 2002; Peinemann et al., 2004). This strongly suggests that aftereffect-duration can be extrapolated from motor to non-motor sites, and vice versa.

None of the reviewed TMS-EEG studies has investigated the time-course of the electrophysiological changes relative to behavioral effects. Based on what is known from behavioral TMS studies, aftereffects of a $1 \mathrm{~Hz}$ protocol would be estimated to last approximately as long as the duration of stimulation (Robertson et al.,
2003). This would have resulted in an estimated 13.6 min vs. a measured $38 \mathrm{~min}$ of aftereffects in the $1 \mathrm{~Hz}$ TMS-EEG studies in which the EEG/EP-aftereffects were assessed ( $n=5$, Supplemental material, Table S2). Although speculative at this point, it is probably safe to conclude that the time of potential aftereffects would be slightly, but not dramatically, underestimated if equated to the duration of observable behavioral effects (using safe parameters).

Less is known for TBS stimulation. Yet, with the previously employed parameters (as compared to standard protocols: similar number of pulses but considerable shorter duration and lower intensity of stimulation), the duration of the effects on EEG activity (measured so far using SEP-amplitude after sensorimotor stimulation) (Katayama and Rothwell, 2007; Ishikawa et al., 2007) seems to be comparable to those after standard repetitive TMS protocols. Recently, 24 healthy volunteers participated in 2 randomized, placebo-controlled, cross-over experiments and underwent continuous TBS (cTBS), intermittent TBS (iTBS), and shamTBS either over the left dorsolateral prefrontal cortex $(n=12$, Figure 8 coil $)$ or the medial prefrontal cortices ( $n=12$, double-cone coil) (Grossheinrich et al., 2009): the only EEG aftereffects were current density changes in the alpha2 band after iTBS of the dorsolateral prefrontal cortex, which remained detectable up to $50 \mathrm{~min}$ after stimulation. However, more is needed in terms of localized (i.e., coherence, synchronization likelihood) or generalized EEG modifications induced by TBS and other patterned rTMS protocols.

In summary, it seems clear that TMS has a robust neurophysioloical effect on EEG/EPs, and that aftereffects can be demonstrated even in the absence of any behavioral effects. The duration of such aftereffects is estimated to be approximately $1 \mathrm{~h}$ following a single session of TMS using current protocols. This should be taken into consideration when planning experiments and when to dismiss the participants. In comparison to the previous guidelines, there is no new EEG result calling for more caution with conventional repetitive protocols $(0.3-20 \mathrm{~Hz})$, neither in healthy participants nor psychiatric or epilepsy patients. Further EEG studies are needed to collect more data on TBS and repeated PAS, as well as in non-psychiatric or non-epilepsy patients, given the incomplete picture we have for these protocols and patient groups (e.g., stroke). Until then, we urge caution regarding potentially long-lasting aftereffects. It should be noted as well, that this conclusion refers only to a single rTMS intervention. Repeated interventions, for example as employed in therapeutic trials of rTMS, may give rise to effects of different duration, but this is largely unknown, and in need of study.

\subsection{Seizures}

Induction of seizures is the most severe acute adverse effect for rTMS. Several cases of accidental seizures induced by rTMS have been reported to date, most in the early days prior to the definition of safety limits. Considering the large number of subjects and patients who have undergone rTMS studies since 1998 (see Fig. 1) and the small number of seizures, we can assert that the risk of rTMS to induce seizures is certainly very low. 
Seizures are caused by hypersynchronized discharges of groups of neurons in the gray matter, mainly due to an imbalance between inhibitory and excitatory synaptic activity in favor of the latter. Seizures can be induced by rTMS when pulses are applied with relatively high-frequencies and short interval periods between trains of stimulation. rTMS might theoretically induce seizures during two different periods associated with stimulation: (1) during or immediately after trains of rTMS and, (2) during the aftereffects due to the modulation of cortical excitability (i.e., kindling effect, see Wassermannn, 1998). Although the first has been seen, there is no evidence that the latter has ever occurred. Indeed, there is no solid evidence for kindling in humans in any situation.

Here we review the cases of accidental seizures during TMS. This is critical to identify predictors associated with induction of seizures, and in turn to analyze the impact of the previous safety guidelines (Wassermannn, 1998) for prevention of seizures. We do not take into account seizures induced during "magnetic seizure therapy", an alternative way to use rTMS to treat pharmaco-resistant depression (Lisanby, 2002) in which special stimulation devices are employed with the aim of inducing a seizure under controlled conditions and with the patient protected by muscle relaxants and anesthestics.

We conducted a systematic review up to December 2008 in which we used as keywords "seizure", "seizures", "transcranial magnetic stimulation", and "TMS". In addition we searched review papers on TMS safety and collected experience from experts in the field. We initially identified 143 articles, which were reviewed, and if a seizure was reported, we collected information on the parameters of stimulation and baseline clinical and demographic characteristics. A total of 16 cases were identified. Seven of these cases were included in the previous 1998 safety guidelines and 9 of them were reported in the following years. We created a framework in which cases were classified based on whether seizures were induced by stimulation outside or within the recommended parameters according to the previous safety guidelines (intensity, frequency and train duration). We then discussed potential factors that may have contributed to the seizures. It should be kept in mind that the 1998 safety guidelines (Wassermannn, 1998) define a combination of rTMS parameters such as frequency, intensity and duration of trains based on a study in healthy controls that used the outcomes of after-discharges and spread of excitation (Pascual-Leone et al., 1993).

Flitman et al. reported an episode of a generalized tonic clonic seizure in a healthy subject using parameters of $120 \%$ of MT, $15 \mathrm{~Hz}$, train duration of $0.75 \mathrm{~s}$, and with variable intervals between trials. This was a study to determine whether linguistic processing can be selectively disrupted with rTMS (Flitman et al., 1998). The very short interval $(0.25 \mathrm{~s})$ between trains was thought to have contributed to this episode, triggering a detailed study on the impact of duration of inter-train intervals on the risk of seizure induction. The findings resulted in the revision of prior guidelines to include interval between trains as another relevant parameter (Chen et al., 1997).

\subsubsection{Seizures that have occurred with rTMS parameters considered safe according to the 1998 safety guidelines}

Four of the new seizures (two following single-pulse and two following rTMS) induced by TMS since publication of the prior guidelines appear to have been induced by "safe" stimulation parameters.

Figiel et al. reported a case of a patient with major depression who developed left focal motor seizures that followed at least $6 \mathrm{~h}$ after the end of stimulation $(100 \%$ of MT, $10 \mathrm{~Hz}$, and train duration of $5 \mathrm{~s}$ ). The use of antidepressant medications might have increased the risk of seizures. In any case, because neurological exam and EEG were normal and this episode was not responsive to anti- epileptic drugs, pseudoseizure was also considered (Figiel et al., 1998). Given the delay between the stimulation and the event, the relationship is also uncertain. This case emphasizes the critical need of careful documentation, monitoring, and evaluation by a trained clinician.

Nowak et al. (2006) reported a case of a generalized tonic clonic seizure in a patient with tinnitus receiving rTMS with parameters of $90 \%$ of MT, $1 \mathrm{~Hz}$ and 580 pulses (Nowak et al., 2006). There were no identifiable factors that may have contributed to this episode of seizure. However, because of clinical features, it has been questioned whether this episode actually was a convulsive syncope rather than a seizure (Epstein, 2006).

Additional to seizures occurring during rTMS, two cases of a generalized tonic clonic seizure following single-pulse TMS have been reported. One, in a patient with multiple sclerosis $(66 \%$ of TMS output) in a study investigating cortical excitability has been reported. In this case, the brain lesions associated with multiple sclerosis and the use of olanzapine might have increased the risk of seizures (Haupts et al., 2004).

Tharayil et al. (2005) reported a generalized tonic clonic seizure in a patient with bipolar depression using single-pulse TMS during motor threshold (MT) assessment. The use of chlorpromazine and lithium, and also family hisory of epilepsy might have increased the risk of seizures (Tharayil et al., 2005).

In summary, three of these four instances of seizures occurred in patients taking pro-epileptogenic medications, and two of the four cases may represent non-epileptic events.

\subsubsection{Seizures that have occurred with rTMS parameters outside 1998 safety guidelines}

Since 1998 there have been four cases of accidental seizures in studies using parameters outside the previous safety guidelines.

Conca et al. (2000) reported a seizure in a patient with major depression in whom rTMS ( $110 \%$ of MT, $20 \mathrm{~Hz}$, train duration $5 \mathrm{~s}$ ) was being used as an add-on treatment (Conca et al., 2000). The extremely brief loss of consciousness in this patient $(8 \mathrm{~s})$ suggests syncope rather than a seizure (see Epstein, 2006).

Bernabeu et al. (2004) reported a seizure in a healthy volunteer (who was using fluoxetine $20 \mathrm{mg}$ ) in a study to investigate the effects of traumatic brain injury on cortical excitability as measured by a variety of TMS protocols (Bernabeu et al., 2004). The parameters in this case were $110 \%$ of MT using $20 \mathrm{~Hz}$ and train duration of $2 \mathrm{~s}$. In addition to these rTMS parameters, fluoxetine itself is known to be potentially pro-convulsant.

Rosa et al. (2004) reported a generalized tonic clonic seizure in a patient with chronic pain using parameters of $100 \%$ of MT, $10 \mathrm{~Hz}$ and train duration of $10 \mathrm{~s}$.

Prikryl and Kucerova (2005) reported a case of generalized tonic clonic seizure during rTMS treatment for major depression. The parameters of stimulation were $110 \%$ of MT using $15 \mathrm{~Hz}$ and train duration of 10 s. There was also a history of sleep deprivation in this patient.

In summary, three of these four instances of seizures occurred in patients taking pro-epileptogenic medications or following sleep-deprivation, and one of the four cases may represent a non-epileptic event.

\subsubsection{Seizures induced by patterned rTMS}

Since the introduction of theta burst stimulation (TBS) by Huang et al. $(2004,2005)$ a review of the literature reveals 49 publications using TBS in normal participants or patients with tinnitus, stroke, movement disorders, or chronic pain. Overall, a total of 741 participants have undergone either continuous or intermittent TBS. A single seizure has occurred (Obermann and Pascual-Leone, 2009) in a 33-year old man healthy control without any risk factors for epilepsy and not taking any medications. Two days prior to the 
event the subject had an overseas flight from London to Boston, and his sleep pattern may have still been altered, though he reported restful nights and no signs of jetlag. TBS was being applied to the left motor cortex with a MagPro X100 stimulator delivering biphasic pulses via a Figure 8 coil (Model MCF-B65) with each wing measuring $8.5 \mathrm{~cm}$. Stimulation intensity was set at $100 \%$ of resting motor threshold. Continuous TBS was applied as 3 pulses at $50 \mathrm{~Hz}$ with $200 \mathrm{~ms}$ intertrain interval for 50 trains (total of 150 pulses). The event, a partial, secondarily generalized tonic-clonic seizure occurred approximately 5-10 s after the completion of the final train of stimulation and lasted for $40 \mathrm{~s}$ with a post-ictal confusion lasting for approximately $25 \mathrm{~min}$. Physical exam, detailed neurologic exam and mental status exam were normal starting $45 \mathrm{~min}$ after the event and remained normal later. Vital signs were stable, and all tests done were unremarkable. CTBS is traditionally thought to suppress cortical activity. However, it is possible that in some individuals cTBS may lead to facilitatory effects. Such paradoxical modulations have been reported for some subjects undergoing slow rTMS as well. Furthermore, since in this case resting motor threshold (rather than active motor threshold) was used to define the cTBS intensity, the subject was at rest prior to the stimulation, and it is possible that in such circumstances cTBS may have increased the subject's cortical excitability rather than decreasing it (Gentner et al., 2008). It should also be noted that most of the published reports of TBS use an intensity of $80 \%$ of Active Motor Threshold while the seizure occurred in a study applying an intensity of $100 \%$ of Resting Motor Threshold (which is approximately equal to $120 \%$ of Active Motor Threshold). This event also highlights the need for an intensity-dosing study with TBS protocols to assess the seizure risk.

\subsubsection{Risk of seizures in epileptic patients and other patient populations}

There were no TMS-linked seizures among 152 patients with epilepsy who underwent weekly rTMS applications at $\leqslant 1 \mathrm{~Hz}$ in the context of the largest trials designed to investigate the potential of inhibitory low-frequency rTMS to reduce seizure frequency (Theodore et al., 2002; Tergau et al., 2003; Fregni et al., 2006a, 2006b; Cantello et al., 2007; Joo et al., 2007; Santiago-Rodríguez et al., 2008).

Furthermore, the use of high frequency/high intensity rTMS was unsuccessful as a non-invasive procedure to activate epileptogenic foci (see Tassinari et al., 2003, for a review) with the exception of a minority of patients with progressive myoclonic epilepsy, who are particularly susceptible to external stimuli.

Two patients with epilepsia partialis continua received highfrequency rTMS with parameters exceeding previous guidelines, without adverse effects: in the former, $100 \mathrm{~Hz}$ rTMS (15 trains at $90 \%$ of maximal output at successively increasing durations ranging from 0.05 to $1.25 \mathrm{~s}$.); in the latter, trains at $100 \%$ of maximal output, $20 \mathrm{~Hz}$ for $4 \mathrm{~s}$ were applied (Rotenberg et al., 2009).

A recent review on the safety of rTMS in epilepsy (Bae et al., 2007 ) indicated a $1.4 \%$ crude per-subject risk to develop a seizure (4 out of 280 patients) and no cases of status epilepticus. Such a low risk in epileptic patients may be due to use antiepileptic drugs, which might have a protective effect against TMS-induced seizure. In some epilepsy patients, a seizure has occurred during rTMS, but this could not unequivocally be assigned to TMS in many cases or occurred at high TMS intensity beyond safety guidelines (Dhuna et al., 1991). Of course, other factors that may increase the likelihood of inducing seizures such as history of seizures, medications that decrease seizure threshold (see later on), or other diseases potentially affecting cortical excitability (e.g., stroke or autism), need to be considered when assessing safety of rTMS treatment. In chronic stroke patients rTMS application for treatment of associated depressive symptoms was safe (Jorge et al., 2004), but rTMS trains which are usually safe for healthy volunteers (at rates of $20-25 \mathrm{~Hz}, 110-130 \%$ of MT are able to induce peripheral manifestations indexing spread of activation at cortical level, thereby potentially increasing the risk of seizures (Lomarev et al., 2007). Therefore, for patients with additional risk, rigorous monitoring is still critical. In such instances, the recommendations made in 1998 for electroencephalographic monitoring and electromyographic monitoring for spread of excitation should be entirely endorsed, along with video recording (if available) of the TMS session to be able to analyze in detail the characteristics of a spell. The involvement of a physician with expertise in the recognition and acute treatment of seizures is still strongly recommended for such instances.

\subsubsection{Sub-clinical EEG abnormalities due to TMS}

Table 2 summarizes those studies in which EEG recordings have been scrutinized for epileptiform activity (mainly spikes and slowwaves) before, during or after repetitive TMS. TMS-induced subclinical EEG abnormalities have been detected on rare occasions in patients but not in healthy volunteers. Of 49 patients suffering from psychiatric disorders and undergoing TMS treatment (many of them for up to 10 days), three cases showed minor transient epileptiform activity during or after TMS. All these patients were stimulated with high-frequency TMS $(10$ or $20 \mathrm{~Hz})$, and stimulation was continued despite the epileptiform EEG activity and no seizure was induced (Boutros et al., 2000).

In 31 epilepsy patients in whom high frequency TMS was employed $(5-100 \mathrm{~Hz})$, no changes in frequency of spikes (Schulze-Bonhage et al., 1999) or even a reduction in frequency or duration was reported (Jennum et al., 1994; Conte et al., 2007). Low frequency TMS ( $n=177$ epilepsy patients, $0.3-1 \mathrm{~Hz}$ ) has been shown to significantly reduce interictal spike frequency or amplitude (Hufnagel and Elger, 1991; Steinhoff et al., 1993; Misawa et al., 2005; Menkes and Gruenthal, 2000; Rossi et al., 2004a,b; Fregni et al., 2005a,b, 2006a,b; Cantello et al., 2007; Joo et al., 2007) and enhancement has been noted only rarely (Hufnagel and Elger, 1991). Even in patients who are withdrawn from antiepileptic medication, changes in interictal spike pattern and seizure induction during TMS seem to be infrequent and in rare cases only not to be coincidental (Schulze-Bonhage et al., 1999; see also Schrader et al., 2004 for review of single-pulse TMS and epilepsy). While there are many studies in psychiatry and epilepsy research, less is known in regards to TMS-induced EEG abnormalities in other patient groups (e.g., stroke: Fregni et al., 2006a,b).

In healthy participants, rTMS studies have yielded negative results as to TMS-induced EEG abnormalities (45 volunteers in 5 publications). Moreover, in one healthy participant in whom a seizure was induced while EEG was recorded, no abnormal pre-seizure EEG activity was observed (Pascual-Leone et al., 1993).

There is one observation of potentially epileptiform activity online to TMS over central leads in a patient that raises concern (Kanno et al., 2001). This is because the transient epileptiform activity occurred during rTMS at very low frequency $(0.25 \mathrm{~Hz})$ and early into the train (4th stimulus) so that it could not be assigned to cumulative pulse effects and seemed to be driven by only a few single pulses. In addition, this activity was likely to be TMSinduced, because it was reproduced during a second TMS session on another day (Kanno et al., 2001). Because this patient suffered from uncontrolled movements of trunk and limb, it is plausible that this patient's motor cortex might have been extremely hyperexcitable as compared to other populations (Kanno et al., 2001). Aside from this observation, however, there is no report of epileptiform activity in the many publications $(n>25)$ that recorded EEG online to single-pulse TMS with a methodological or fundamental 
neuroscientific perspective (Bridgers and Delaney, 1989; Kujirai et al., 1993; Nikouline et al., 1999; Tiitinen et al., 1999; Paus et al., 2001; Schürmann et al., 2001; Kähkönen et al., 2001, 2004, 2005; Komssi et al., 2002, 2004; Kübler et al., 2002; Thut et al., 2003a,b; Thut et al., 2005; Massimini et al., 2005, 2007; Price, 2004; Fuggetta et al., 2005, 2006; Bonato et al., 2006; Van Der Werf et al., 2006; Van Der Werf and Paus, 2006; Morbidi et al., 2007; Litvak et al., 2007; Julkunen et al., 2008; Romei et al., 2008a,b).

Therefore, it seems that subclinical epileptiform EEG activity during conventional rTMS is overall very rare, and that EEG monitoring before and during rTMS cannot effectively prevent accidental seizures induction.

\subsection{Syncope}

Vasodepressor (neurocardiogenic) syncope is a common reaction to anxiety and psycho-physical discomfort. It is a common experience that may occur more often than epileptic seizures during TMS testing and treatment, including TBS (Grossheinrich et al., 2009), as along with many other medical procedures (Lin et al., 1982).

Diagnostic problems arise in subjects who manifest positive phenomena during a syncopal episode. These may include behaviors considered typical of seizures: tonic stiffening, jerking, vocalizations, oral and motor automatisms, brief head or eye version, incontinence, hallucinations, and injuries from falling. Such episodes can be difficult to distinguish from epileptic events, although tongue biting and loss of urine are often lacking. Clinically, the cardinal feature that distinguishes syncope from seizure is rapid recovery of consciousness within seconds and not minutes (Lin et al., 1982). The premonitory complaint that "I need to lie down", or "I need air", narrowing and blacking out of the visual field, sensations of heat, bradycardia, and loss of peripheral pulses also favor circulatory collapse. Visceral distress, nausea, dizziness, pallor, and diaphoresis are frequent symptoms (Adams and Victor, 1977). Gastrointestinal symptoms occur in partial epilepsies as well, but their incidence in seizures provoked by TMS is unclear. Upward eye deviation is common in circulatory syncope, but rare in partial seizures unless they progress to generalized convulsions.

EKG, EEG, and video recording can provide important diagnostic information when available (Lempert, 1996). Elevated prolactin, drawn within $15 \mathrm{~min}$ of an event, can document the occurrence of generalized seizure, but a normal prolactin has no diagnostic value. The web site of the journal Brain Stimulation (www.brainstimjrnl.com/content/mmc_library) contains a video (courtesy of Thomas Lempert) showing the occurrence of a seizure and a syncope, which might be useful for didactic puposes. This journal has a new rapid letter to the editor format (http://ees.elsevier.com/brs/) to encourage rapid reporting of all TMS induced seizures.

Initial measures for suspected seizures and syncope are identical. TMS should be terminated immediately, and the subject assisted in controlled reclining without impact. Airway breathing and circulation should be assessed. Unless tonic-clonic seizure activity occurs, the subject should be turned on one side to help clear the airway and avoid aspiration. Subjects who convulse should be turned on one side as soon as movement ceases, and maintained in that position until recovery of awareness. Delayed recovery of normal consciousness beyond $30 \mathrm{~s}$ following a seizure mandates further medical evaluation.

\subsection{Local pain, headache, discomfort}

Single-pulse TMS is generally well tolerated and experienced by most participants as painless. Some discomfort can be induced when using the triple stimulation technique (Magistris et al.
1998, 1999) due to the associated, supra-threshold peripheral stimulation. At times TMS and especially rTMS can be painful. This is in fact the most common side-effect of TMS. The intensity of pain experienced varies from subject to subject, depending on individual susceptibility, scalp location, coil design, intensity and frequency of stimulation. Patients and subjects should be warned that TMS may not be pleasant and may cause pain.

In a recent metanalysis on the safety of TMS for depression (Loo et al., 2008), that took into account all sham-controlled studies reporting specifically side-effects, about $28 \%$ of patients experienced headache and $39 \%$ experienced pain or discomfort during active rTMS, compared with rates of side-effects of $16 \%$ and $15 \%$, respectively after sham rTMS. Another review reported headache or neck pain as the most frequent (i.e., about $40 \%$ of cases) side effect of rTMS applied to non-motor areas (Machii et al., 2006). So, depending on the research question and design, this level of pain should also be matched in an active sham condition. Mimicking the cutaneous sensation experienced during rTMS has been a challenging aspect of developing an optimal sham condition (see Ruohonen et al., 2000; Lisanby et al., 2001; Sommer et al., 2006a; Rossi et al., 2007b; Arana et al., 2008). The cutaneous sensation is caused when rTMS stimulates scalp muscles producing a twitch in the scalp or upper face that can be uncomfortable for some, painful for others. However, this aspect in terms of safety is negligible.

In the clinical trials of TMS to date, only a small percentage of patients have discontinued treatment due to pain $(<2 \%)$. Some researchers have studied options to reduce the painfulness of TMS treatment, including topical anesthetics (EMLA, Eutetic mixture of local anesthetics, cream), local injection of $1 \%$ lidocaine with or without epinephrine, and insertion of thin air-filled styrofoam pads (Bockart et al., 2008). There was no change in pain rating with the EMLA cream, only a 7\% reduction with the foam pad, but a $50 \%$ reduction in pain intensity with lidocaine injection. However, in some subjects subsequent hypersensitivity occurred. One manufacturer has also developed a thin electric pad insert that is supposed to modify the TMS magnetic field and reduce pain. This system has been reported to fail once resulting in a minor burn to the patient (O'Reardon et al., 2007).

It is unclear exactly what causes the painfulness of TMS, although it is likely that trigeminal stimulation plays a role. TMS induced muscle contraction can be relevant, particularly away from the vertex. Neck pain is likely related to the forced posture and head immobilization during the rTMS session (Machii et al., 2006). Occasionally, neck pain can be associated with nausea after cerebellar stimulation (Satow et al., 2002).

In the majority of subjects/patients experiencing local pain during TMS, including toothache, the effect rapidly vanishes. Headache may occasionally persist, however, after TMS application; in this case, a common analgesic administered orally may be helpful. No migraine attacks have been described following rTMS, neither in normals nor in migraine patients who underwent rTMS applications as treatment (Brighina et al., 2004). It has been reported that the local painfulness of prefrontal rTMS declines over the first few days of daily treatment (Janicak et al., 2008; Anderson et al., 2009). With this knowledge, some TMS treatment trials incorporate a ramping up algorithm, intentionally starting below target dose and gradually increasing this over the first week of treatment.

\subsection{Cognitive/neuropsychological changes}

\subsubsection{Single or repeated trains applied for physiological or cognitive} brain research

In the cognitive domain, TMS can produce desired (usually within the frame of the experimental design) and undesired, potentially long lasting, changes. In the former, TMS can make subjects perform better or worse on a given task, generally in the 
form of percentage of correct responses, reaction time or signal detection measures. Such effects, which generally follow a single application of rTMS, can be produced by random neural noise or brain signal suppression (Harris et al., 2008), are small and, reasonably, do not raise particular safety issues (see Rossi et al., 2007c).

In several studies, certain cognitive tasks have been demonstrated even to be enhanced by rTMS revealing the potential of TMS-induced paradoxical functional facilitation (Theoret et al., 2003). Changes are reported to be small and only temporary, although the time-course has not been followed in detail in all studies. The mechanisms of cognitive enhancement are not understood and remain an intriguing area of research. With the caveat that observation in the motor system may not be applicable to other domains of behavior these mechanisms may include local enhancement of neuronal excitability (changes of intracortical inhibition, ionic membrane conductance, changes of synaptic efficacy) and network effects (release of remote inhibition, change of balance). Indeed, rTMS applied to Wernicke's or Broca's area led, respectively, to inhibition or facilitation of language (Dräger et al., 2004). The same subthreshold rTMS protocol applied to the same prefrontal brain regions have led to facilitation of action naming in one study (Cappa et al. 2002) and inhibition of episodic encoding in others (Rossi et al., 2001, 2004a,b, 2006). These observations suggest that the same protocol may induce opposite effects in slightly different circumstances, and that no extrapolation may be made from one cortical region to another or from one cognitive function to a related one.

We performed a survey of the studies that have used TMS trains to interact with task performance in cognitive science from 1999 to December 2008. In these protocols, short trains of a few hundreds of ms are applied online to task performance on the sub-trial level (usually aligned to trial onset), in order to cover much of the time-interval between sensory input and motor output in which task-related cognitive processing is expected to occur. These protocols have been interchangeably referred to, for instance, as online rTMS interference approach, or triple-pulse TMS for trains of three pulses, among other nomenclature. We propose to use the operationally defined terminology of online rTMS interaction protocols.

These protocols have been widely employed in healthy volunteers without side-effects, following the publication of the previous 1998 safety guidelines (Wassermannn, 1998) and after screening via the safety questionnaire to eliminate contraindications (see Section 7.5). This has resulted in a large collection of empirical data for TMS applications beyond single-pulse, doublepulse and 1-Hz TMS in psychology and cognitive sciences (see Supplemental material, Table S3).

Over the last 10 years, $4 \mathrm{~Hz}$ - to $25 \mathrm{~Hz}$-trains have been tailored to cover usually $0.1-1 \mathrm{~s}$ and exceptionally up to $30 \mathrm{~s}$ of task performance. More than 50 studies used $10 \mathrm{~Hz}$, more than 20 have employed $20-25 \mathrm{~Hz}$ and more than 10 studies used $4-9 \mathrm{~Hz}$. Parameters to consider for designing experiments are the duration of the TMS-train, the stimulation rate (in $\mathrm{Hz}$ ), the inter-train interval and the number of trials within the experiment (summarized in Supplemental material, Table S3). For safety aspects, the combination of parameters is important, with short train durations and long inter-train intervals carrying less risk (Table 3).
The most frequently used parameter-combination for $10 \mathrm{~Hz}$ protocols has been 5-6 pulse-trains for $400-500 \mathrm{~ms}$, applied with a mean inter-train interval of $3.2 \mathrm{~s}$ over an average of 250 trials. The most frequently used parameter-combination for $20-25 \mathrm{~Hz}$ protocols has been $10-11$ pulse-trains for $400-500 \mathrm{~ms}$, applied with a mean inter-train interval of $17.1 \mathrm{~s}$ over an average of 80 trials. Parameter combinations for $4-9 \mathrm{~Hz}$ applications have been more variable. Applications with longer train durations (>500 ms) have been used with $4-20 \mathrm{~Hz}$ without side-effects (see Table 3). For $10 \mathrm{~Hz}$ protocols, those that applied the highest number of pulses within a train ( $n=30$ for $3 \mathrm{~s}$ ), also employed long inter-train intervals (10-12.5 s; see Supplemental material, Table S3), as was the case for $20-25 \mathrm{~Hz}$ application ( $n=20$ pulses for $1 \mathrm{~s}$, at least $10 \mathrm{~s}$ inter-train interval; Supplemental material, Table S3).

To avoid possible side-effects also in the future and to remain within safe margins, future studies using the online interaction protocols could design their parameters to fall within the range summarized here (Table 3, see also Supplemental material, Table S3).

\subsection{2. rTMS for therapy}

Possible hazards regarding long-lasting cognitive changes are related to the cumulative effects of repeated sessions of rTMS, in the frame of therapeutic applications for neurological and, mainly, psychiatric diseases. Two recent articles examined extensively this issue: in a meta-analysis of 173 papers published from January 1998 to December 2003 (more than 3000 subjects/patients) that reported application of rTMS to non-motor areas, side-effects vaguely related to cognition included excessive tiredness, concentration difficulties, memory difficulties and were reported to be mild, transient and to be "very rare" (see Table 4 of Machii et al., 2006). Another comprehensive review of sham-controlled rTMS studies in major depression identified 39 studies (more than 1200 patients overall), of whom 12 reported cognitive improvement. In 3 studies at least 1 cognitive test deteriorated, among vast batteries of cognitive examinations, while improvements were noted in other tests (Loo et al., 2008). Therefore, unequivocal unintentional cognitive deterioration has not been noted in any studies.

One issue particularly relevant to studies of psychiatric patients is mood changes (Loo et al., 2008). Even when such changes were induced in patients, no correlation emerged between mood and cognitive performance.

While most studies have focused on static cognitive functions, others have shown that rTMS may modulate the acquisition of new skills or memory. rTMS-induced changes have been found to last in the order of several tens of minutes maximally. However, the possibility of very-long $(>24 \mathrm{~h}$ ) lasting stimulation-induced cognitive changes cannot be entirely excluded.

Cognitive function was additionally assessed in one large multicenter sponsored study examining the effect of rTMS (120\% MT, $10 \mathrm{~Hz}, 3000$ pulses, maximal number of total pulses 216,000 ) in 325 patients with major depression by three different tests (Mini Mental State Examination, Buschke selective reminding test, Autobiographical memory interview). No cognitive changes were noted (Janicak et al., 2008). In another recent study, massed application of max 38,880 magnetic pulses over 1 week did not lead to cognitive changes (Anderson et al., 2006). However, absence of cognitive

Table 3

Summary of the most employed average stimulation parameters in online interaction rTMS protocols and found to be safe. Consensus has been reached for this table.

\begin{tabular}{|c|c|c|c|c|}
\hline rTMS frequency & Number of studies & Average train duration & Average inter-train interval & Average number of trials \\
\hline 4-9 Hz & $>10$ & \multicolumn{3}{|c|}{ Variable (see Supplemental material, Table S3) } \\
\hline $10 \mathrm{~Hz}$ & $>50$ & $5-6$ pulse-trains for $400-500 \mathrm{~ms}$ & $3.2 \mathrm{~s}$ & 250 \\
\hline $20-25 \mathrm{~Hz}$ & $>20$ & 10 pulse-trains for $400-500 \mathrm{~ms}$ & $17.1 \mathrm{~s}$ & 80 \\
\hline
\end{tabular}


changes must not be taken as evidence of absence of changes in neuronal function. Experiments using functional imaging and dual-site TMS have revealed unequivocal evidence of extremely rapid plasticity (e.g., O'Shea et al., 2007), which may mask local interference effects.

As emphasized already, one issue particularly pertinent to rTMS-protocols in therapeutic sessions is the question of whether multiple sessions, performed within a short time span such as over 1 week, will lead to cumulative effects. In the visuo-spatial domain, such phenomena have been demonstrated in cats (Valero-Cabré et al., 2008), when rTMS stimulations were applied on a daily basis, whereas previous studies with rTMS sessions spaced apart by $48 \mathrm{~h}$ failed to produce such effects. Studies in the motor system in humans have reported a similar phenomenon, demonstrating that cumulative effects have to be considered in humans also (Lomarev et al., 2006).

Among the "new" protocols of brain stimulation such as TBS, QPS and PAS, only the former has been used to intentionally modulate cognitive capacities. Its efficacy to induce cognitive changes appears to be somewhat weaker than $1-\mathrm{Hz}$ rTMS, at least in the motor cortex (Mistry et al., 2007), although both protocols were not compared head-to-head in the same study. The duration of TBS-induced cognitive changes does not appear to differ substantially from 1-Hz rTMS and lies in the range of several tens of minutes. However, further studies on this issue are needed.

The methodology used for the application of rTMS is such that it may easily induce a placebo effect in some patients. This effect, as it is the case with other medical interventions, may add to the effects induced through activation of neural structures and may contribute to the subjective feeling of improvement in some instances. Although many studies included sham-stimulation as control, there is nowadays no guarantee that a true sham-rTMS condition is available and, therefore, the relative size of the placebo effect may be difficult to determine in any rTMS treatment. This is likely to be more difficult when testing functions depending on subjective assessment than when testing quantifiable changes (Fregni et al., 2006a,b).

\subsection{Acute psychiatric changes}

Treatment-emergent mania has been reported for low and high frequency rTMS in patients with uni- and bipolar depression (Xia et al., 2008) after stimulation of the left prefrontal cortex. Although single cases suggest a causal relationship between rTMS and mania, the overall rate (13 cases) across 53 randomized controlled studies in depression appears to be low (0.84\% mania for active rTMS vs. $0.73 \%$ for sham rTMS) and even below natural switch rates in patients with bipolar disorders receiving mood stabilizers (2.3-3.45\%) (Xia et al., 2008).

Similarly, cases of rTMS induced psychotic symptoms, anxiety, agitation, suicidal ideation and insomnia (Zwanzger et al., 2002;

\section{Table 4}

Maximum safe duration (expressed in seconds) of single trains of rTMS. Safety defined as absence of seizure, spread of excitation or afterdischarge of EMG activity. Numbers preceded by > are longest duration tested. Consensus has been reached for this table.

\begin{tabular}{clllll}
\hline Frequency $(\mathrm{Hz})$ & \multicolumn{5}{l}{ Intensity $(\%$ of MT) } \\
\cline { 2 - 6 } & $90 \%$ & $100 \%$ & $110 \%$ & $120 \%$ & $130 \%$ \\
\hline 1 & $>1800^{\mathrm{a}}$ & $>1800$ & $>1800$ & $>360$ & $>50$ \\
5 & $>10$ & $>10$ & $>10$ & $>10$ & $>10$ \\
10 & $>5$ & $>5$ & $>5$ & 4.2 & 2.9 \\
20 & 2.05 & 2.05 & 1.6 & 1.0 & 0.55 \\
25 & 1.28 & 1.28 & 0.84 & 0.4 & 0.24 \\
\hline
\end{tabular}

\footnotetext{
a In Japan, up to 5000 pulses have been applied without safety problems (communication of Y. Ugawa).
}

Janicak et al., 2008) have been reported, but it is unknown whether these occur at higher rates compared to the natural course of disease being treated or associated with other interventions. Psychotic symptoms and suicidal ideation have been never described in normal subjects during or after rTMS.

In all the above cases the psychiatric side effects induced by TMS were transient, with a spontaneous resolution after TMS cessation or promptly controlled by pharmacological treatment. Nevertheless, patients with depression undergoing rTMS should be informed about the unlikely possibility of developing such acute side effects depending on type and severity of disease.

In 24 normal subjects who underwent different TBS protocols to dorsolateral and medial prefrontal cortex no mood changes were noted (Grossheinrich et al., 2009).

Whereas these acute side effects are relatively well characterized, there is no systematic research on cumulative side effects which may occur when cumulative "therapeutic" aftereffects are expected, with the exception of one sponsored study that did not document late-appearing adverse events for up to 6 months after the acute clinical benefit (Janicak et al., 2008).

\subsection{Other biological effects possibly related to safety concerns}

\subsubsection{The need of animal models}

Given the increase in TMS use in neuroscience research and progressive mainstream spread of TMS based-therapeutic approaches, the use of adequate animal models to pre-assess acute and longterm safety would be extremely valuable. A useful animal model needs to allow for the combination of a precise and reliable stimulation method with monitoring tools of high spatial and temporal resolution to capture the physiologic impact. Monitoring methods of high spatial resolution include metabolic/pharmacologic labeling, optical imaging, and high-field fMRI. Monitoring methods of high temporal resolution are field- or single-unit electrophysiological recording. Ideally, both of these types of monitoring methods should be combined and applied simultaneously. Furthermore, an ideal animal model should allow for the exploration of the behavioral correlates of the stimulation in the awake freely moving animal. A pre-existing knowledge on the anatomical connectivity between regions and the effects of other types of brain manipulation in the same regions, such as lesion studies, pharmacologic deactivations, microstimulation or cooling deactivations is obviously helpful in the interpretation of the results.

Rodents, felines, and in a very limited fashion non-human primates have been all used in TMS studies aimed at understanding the physiology underlying its effects. However, the ratio between head size and coil size remains the main issue precluding an easy interpretation and transferability of animal results into human applications, since the induced current density distribution and the spatial selectivity of the impact is strongly affected by the thickness and size of the brain (Post and Keck, 2001). This is particularly critical for rodent models, in which spatially selective repetitive stimulation of specific neural networks requires specially designed smaller TMS coils (Luft et al., 2001). The use of standard coils, like in early studies, raises two types of problems. The first one is a loss of stimulation specificity, and in most cases the whole brain or even the body of the animal is stimulated with the risk of inducing irrelevant results. Second, and most important, is that the efficiency of magnetic stimulation is drastically reduced in smaller brains (Weissman et al., 1992) since rTMS-induced current depends, besides brain size, by conductivity of brain structure. Therefore the direct comparison based only on frequency and intensity of stimulation used in some animal studies may not be valid for humans.

The issue of brain tissue heating due to TMS has been addressed in Section 3 of the current manuscript. As reviewed by Post and 
Keck (2001) concerning possible hazardous effects of rTMS at the structural and cellular level in the brain, Matsumiya et al. (1992) reported microvacuolar changes in the neuropil portion of cortical layers 2-6 in rats stimulated with 2.8 Tesla for at least 100 stimuli, but these may have resulted from TMS-induced head jerking or may even represent freezing artifacts. In contrast, Sgro et al. (1991) did not detect significant morphological changes in the various rat brain regions after rTMS for at least 10,000 stimulations with 3.4 Tesla, nor did Counter (1993) after chronic low frequency TMS (2.0 Tesla; 1000 stimuli) of rabbit brains. The safety of chronic rTMS was recently evaluated in vivo by using localized proton magnetic resonance spectroscopy and post mortem histological analysis (Liebetanz et al., 2003). In this study, conscious rats were treated with a daily suprathreshold rTMS regimen of $10001 \mathrm{~Hz}$ stimuli applied on 5 consecutive days in comparison with control animals receiving TMS over the lumbar spine. Spectroscopy did not reveal any change of $\mathrm{N}$-acetyl-aspartate, creatine and phosphocreatine, choline-containing compounds, myoinositol, glucose and lactate after chronic rTMS. Similarly to the in vivo results, post mortem histology revealed no changes in microglial and astrocytic activation after rTMS (Liebetanz et al., 2003).

A study of the neuroanatomical effects of chronic exposure to high dosages of rTMS given at convulsive levels $(50 \mathrm{~Hz}, 8 \mathrm{~s}$ trains, $100 \%$ maximal stimulator output, for 5 weeks) to rhesus macaques revealed no evidence of neuropathological damage (Dwork et al., 2004).

\subsubsection{Endocrine after-effects}

An important safety aspect of rTMS (and a potential factor explaining part of its physiological effects), is the response of hormones of the hypothalamic-pituitary axis such as prolactin, thyroid-stimulating hormone (TSH), follicle-stimulating hormone (FSH) and cortisol. Some studies have addressed this issue and in general most of them, which were not controlled and without a placebo condition, showed no impact of the stimulation on the plasma levels of central hormones except a decline of prolactin during the study procedure (Bridgers and Delaney, 1989; Wassermannn et al., 1996). In one study on 10 healthy volunteers, George et al. (1996) observed an increase of TSH after rTMS of frontal cortex with all other hormones remaining unaffected. Transient increase of TSH release was subsequently reported in a doubleblind study on 14 medication-free subjects with major depression who received individual sessions of either active or sham rTMS (left prefrontal cortex, $10 \mathrm{~Hz}, 100 \%$ of MT, 20 trains over $10 \mathrm{~min}$ ) (Szuba et al., 2001).

Evers and associates have looked at the impact of rTMS on neuroendocrinological serum levels by a placebo-controlled cross-over study (Evers et al., 2001a,b). rTMS was employed in a typical paradigm used in the treatment of depression (coil placed over left dorsolateral prefrontal cortex, 10 and $20 \mathrm{~Hz}$ stimulation). Placebo, infrathreshold, and suprathreshold stimulation were applied in random order. The serum levels of cortisol, prolactin, FSH, and TSH were measured before and after stimulation. After infrathreshold stimulation, cortisol and TSH serum levels decreased mildly, but significantly. All other stimulations had no significant impact on hormone levels. Considering that cortisol and TSH are released in stressful situations the decrease of these hormones suggest a relaxing effect by this type of rTMS in healthy subjects.

Neuroactive steroids progesterone, 3alpha-5alpha-tetrahydroprogesterone, 3alpha-5beta-tetrahydroprogesterone, 3beta-5alpha-tetrahydroprogesterone and dehydroepiandrosterone were quantified in 37 medication-free patients suffering from a major depressive episode before and after 10 sessions of left prefrontal rTMS (Padberg et al., 2002). Plasma concentrations of these neuroactive steroids were not affected by rTMS and not related to the beneficial clinical response.

\subsubsection{Histotoxicity}

Studies addressing structural changes in humans are few. A study of a resected human temporal lobe that had been exposed to rTMS revealed no histopathological changes (Gates et al., 1992). Imaging studies have not shown structural changes after rTMS treatment (Nahas et al., 2000). Diffusion-weighted MRI, which is particularly sensitive to brain tissue damage, has been used to investigate rTMS-induced structural changes, but at the moment with inconsistent results (see Li et al., 2003; Mottaghy et al., 2003; Duning et al., 2004). More recently $1 \mathrm{~Hz}$ rTMS delivered to the superior temporal cortex daily for 5 consecutive days was found to induce bilateral grey matter changes in the auditory cortex (May et al., 2007). However, the significance of these changes remains unclear. First, the findings resolved within a few days of follow-up. Second, from a methodological point of view, increased thickness of the gray matter may have represented expansion of extracellular fluid and blood flow. Although the interpretation of these few studies remains uncertain, it seems reasonable that investigators and patients should be aware of the possibility of cumulative side effects, but further morphometric studies are obviously desirable.

\subsubsection{Effects on neurotransmitters}

Repetitive TMS can acutely affect several neurotransmitters. More studies are needed to fully understand such effects and assess their potential relevance for the mechanisms of action and safety of TMS.

Frontal lobe stimulation at $20 \mathrm{~Hz}$ induced a marked increase of dopamine in the hippocampus (Keck et al., 2000). rTMS of the left dorsolateral prefrontal cortex caused a reduction in [(11)C]raclopride binding in the left dorsal caudate nucleus compared with rTMS of the left occipital cortex indicating increased dopamine release (Strafella et al., 2001). Theoretically, these acute effects on dopaminergic system may induce some beneficial effects on parkinsonian symptoms (Lefaucheur et al., 2004) or psychiatric side effects, like acute mania, particularly in patients with pre-existent abnormalities in the fronto-parietal circuits.

Stimulation of the left dorsolateral prefrontal cortex $(20 \mathrm{~Hz}$, $20 \mathrm{~min} /$ day) may affect cortical glutamate/glutamine levels, not only close to the stimulation site, but also in remote (right dorsolateral prefrontal cortex, left cingulate cortex) brain regions (Michael et al., 2003). Acute $10 \mathrm{~Hz}$ rTMS of the left dorsolateral prefrontal cortex may modulate tryptophan/5-HT metabolism in limbic areas in normal subjects without inducing behavioral changes (Sibon et al., 2007).

There are preliminary data showing that high frequency rTMS $(20 \mathrm{~Hz})$ may activate c-fos transcription (a marker of neuronal activation) in neurons in layers I-IV and VI of parietal cortex and in few neurons of the hippocampus (Hausmann et al., 2001). Investigations on the long-term effects of rTMS treatments are under way.

Recently, Alagona et al. (2009) suggested that both $1 \mathrm{~Hz}$ and $20 \mathrm{~Hz}$ delivered by a round coil on the vertex for $15 \mathrm{~min}$ (900 and 18,000 stimuli, respectively) at $90 \%$ of resting MT induced increased blood levels of lactate of central origin, probably released by astrocyte activity, whose significance is still unceartain. Moreover, it is unclear how round coil heating during $15 \mathrm{~min}$ of $20 \mathrm{~Hz}$ stimulation (a combination that exceeds safety guidelines) was prevented in this study. When repeating this paradigm with an ice-cooled standard round coil the stimulator stopped due to overheating after 3000 stimuli (tecnical experiment without application of the protocol in human subjects, $\mathrm{H}$. Rothkegel and $\mathrm{W}$. Paulus, personal communication).

\subsubsection{Effects on the immune system}

There are several lines of evidence suggesting a lateralized cortical regulatory influence on immune function in humans: left 
hemisphere stimulation can increase whereas right hemisphere stimulation can decrease circulating lymphocyte numbers. In addition crude lesions of the right or left neocortex of rodents has been shown to induce opposite effects on mitogen-induced lymphoproliferation, natural killer cell activity, macrophage activation and interleukin-2 production (Neveu et al., 1989). Nevertheless there are only few papers which have addressed the after-effects of rTMS on immune system, and there are no definite influences that are well accepted at this time. TMS has been used to investigate asymmetrical cortical regulatory influences on one aspect of immune function: secretion of the antibody immunoglobulin A (S-IgA) into saliva. An increase of S-IgA was apparent following left but not right hemisphere stimulation (Clow et al., 2003). Studies on the impact of rTMS on immune function could have potential relevance for the safety of rTMS and warrant further investigation.

\subsubsection{Autonomic function}

Only few papers have looked at the aftereffects of rTMS on autonomic system, despite the fact that many brain areas are implicated in blood pressure, breathing characteristics and heart rate control (see Filippi et al., 2000), and that such effects of rTMS could have obvious safety implications.

Repetitive TMS over several scalp positions $(500 \mathrm{~ms}, 20 \mathrm{~Hz}, 70-$ $90 \%$ of max output) can induce a short lasting increase in heart rate and blood pressure (Foerster et al., 1997). In another study it was shown that rTMS at a low rate can influence autonomic function, evaluated by heart rate variability (Yoshida et al., 2001). This was not the case following acute $10 \mathrm{~Hz}$ rTMS of the left dorsolateral prefrontal cortex (Sibon et al., 2007).

Udupa et al. (2007) treated a group of 27 depressed patients by rTMS of the left PFC with 12 sessions (10 trains of $10 \mathrm{~s}, 15 \mathrm{~Hz}, 60 \mathrm{~s}$ inter-trial interval, $100 \% \mathrm{MT}$ ) of stimulation over a 2 -week period, for a total of 18.000 stimulations. Heart rate variability measures indicated that rTMS produced significantly greater reduction than serotonergic agents (taken by a second group of 25 patients) in the sympathetic/parasympathetic ratio, suggesting improvement in sympathovagal balance. No deleterious effects were noted.

A recent study has investigated the effect of rTMS on cerebral hemodynamics, which might be modified by an effect on autonomic control. Twenty-nine healthy subjects were randomly assigned to real (19) or sham $17-\mathrm{Hz}$ rTMS, applied on primary motor cortex of the dominant hemisphere. All subjects underwent Transcranial Doppler of the middle cerebral arteries to evaluate mean flow velocity and vaso-motor-reactivity (VMR) before (baseline) and within $10 \mathrm{~min}$ following rTMS. Four subjects underwent further VMR evaluations at 2, 5 and $24 \mathrm{~h}$ after rTMS. As a control condition, 10 subjects underwent real or sham rTMS on calcarine cortex. In addition, five acute stroke patients underwent five daily rTMS sessions on the affected hemisphere mimicking a therapeutic trial. Following real rTMS on motor and calcarine cortex VMR decreased significantly in both hemispheres, while no change was observed after sham rTMS. VMR tended to remain lower than baseline for $5 \mathrm{~h}$. Cerebral VMR decreased independently of the stimulated side also in the patients' group. It was concluded that rTMS can reduce cerebral VMR, possibly as a secondary effect on autonomic control of cerebral hemodynamics (Vernieri et al., 2009). This observation should be taken into account when applying rTMS protocols in acute stroke patients.

\section{Considerations on patient selection}

The following paragraphs include several issues that should be taken into account (and should be developed in terms of future specific research) before planning a therapeutic course with rTMS, as well as pathophysiological group studies in patients.
To understand the special safety and ethical issues presented by therapeutic applications of TMS, it is important to consider the potential impact of the neurological or psychiatric illness under treatment, the impact of concomitant treatments, and the chronicity of exposure. Applications of TMS in patients populations differ from basic neuroscience applications in that the subject receiving the TMS has an existing neurological or psychiatric illness, and is likely to be receiving concomitant treatments with a range of CNS acting medications (e.g., antidepressants, antipsychotics, anxiolytics, analgesics, anticonvulsants) that may affect the action of TMS and affect seizure risk.

\subsection{Illness-stimulation interactions}

The manifestations, symptoms, and disability of an illness represent a combination of stable traits and variable state features that may alter the action of TMS. The effects of TMS will likely vary if applied in the acute phase of the illness or for relapse prevention following recovery.

Since effects of rTMS are dependent on the state of activation of the targeted cortex (see Section 5.4), it is salient to consider the effect of the illness on the basal level of activation of the targeted cortex. For example, depression causes alterations in functional activation of the dorsolateral prefrontal cortex (often decreases, though there is significant individual variation in the laterality and direction of these effects). Kimbrell et al. (1999) found the basal level of activation in dorsolateral prefrontal cortex to predict the likelihood of therapeutic response to rTMS to that region. Such relationships might be expected not only for therapeutic effects but also for side effects. Given that rTMS exerts trans-synaptic effects, the impact of illness on functional connectivity may be expected to influence the degree and nature of these distal effects. Studies have shown rTMS to exert neuroplastic effects hypothesized to be mediated via homo- or hetero-synaptic plasticity, depending upon the paradigm used. Illnesses that alter the neurochemistry underlying the acquisition of plasticity (e.g., altered glutamatergic function in schizophrenia) may be expected to alter the response to rTMS. Indeed, Fitzgerald et al. (2004) found schizophrenic patients to show an abnormally dampened neuroplastic response to $1 \mathrm{~Hz}$ rTMS.

Other potential interactions between illness and stimulation include illness-specific side effects (such as increased risk for mania in bipolar patients, or increased risk of seizure in the presence of cerebrovascular lesions). Clinical populations typically have comorbid diagnoses that may further confound the expected effects (and side-effects) of stimulation.

A special risk is occult substance abuse or dependence (alcohol, caffeine, drugs) conditions associated with altered seizure risk. Finally, structural brain changes related to the underlying pathology (e.g., brain atrophy in dementing illnesses) may alter the current distribution and thus influence the effects and safety of TMS (see Section 2.2).

\subsection{Interactions between concomitant treatments and rTMS}

Clinical populations may be expected to be receiving other forms of therapy, such as psychotherapy, neurorehabilitation, and medications. Typically psychotherapy would be administered remotely from rTMS exposure; however, the potential impact of simultaneous delivery on response and side effects is relatively unexplored. The potential of rTMS to prime response to neurorehabilitation is being examined in cerebrovascular disease.

However, the primary safety concern for an interaction between rTMS and concomitant treatment is centrally acting medications. Several antidepressants and neuroleptics increase seizure 
risk (see the following section), while anticonvulsants lower it (see Section 4.2). Patients may be on a combination of agents with synergistic or opposing effects on seizure risk. The net effect on seizure risk of rTMS in such cases has not been systematically studied.

\subsection{Drugs which are potential hazards for rTMS}

Intake of or withdrawal from certain central nervous system (CNS) active drugs lowers seizure threshold. The actual risk for seizure induction may depend on additional, not yet fully explored, factors such as drug dose, speed of dose increase (or decrease), and combination with other CNS active drugs. As discussed in Section 4.3, the majority of reported rTMS-induced seizures have occurred in subjects/patients on drugs with seizure threshold lowering potential. We provide a list of drugs that, on the basis of the current knowledge, form strong or simply relative hazards for the use of rTMS. These lists are by no means meant to be complete and may be amended whenever further knowledge becomes available:

(1) Intake of one or a combination of the following drugs forms a strong potential hazard for application of rTMS due to their significant seizure threshold lowering potential: imipramine, amitriptyline, doxepine, nortriptyline, maprotiline, chlorpromazine, clozapine, foscarnet, ganciclovir, ritonavir, amphetamines, cocaine, (MDMA, ecstasy), phencyclidine (PCP, angel's dust), ketamine, gamma-hydroxybutyrate (GHB), alcohol, theophylline. In these cases rTMS should be performed, when required, with particular caution.

(2) Intake of one or a combination of the following drugs forms a relative hazard for application of rTMS due to their significant seizure threshold lowering potential: mianserin, fluoxetine, fluvoxamine, paroxetine, sertraline, citalopram, reboxetine, venlafaxine, duloxetine, bupropion, mirtazapine, fluphenazine, pimozide, haloperidol, olanzapine, quetiapine, aripiprazole, ziprasidone, risperidone, chloroquine, mefloquine, imipenem, penicillin, ampicillin, cephalosporins, metronidazole, isoniazid, levofloxacin, cyclosporin, chlorambucil, vincristine, methotrexate, cytosine arabinoside, BCNU, lithium, anticholinergics, antihistamines, sympathomimetics. In these cases rTMS should be performed, when required, with caution.

(3) Withdrawal from one of the following drugs forms a strong relative hazard for application of rTMS due to the resulting significant seizure threshold lowering potential: alcohol, barbiturates, benzodiazepines, meprobamate, chloral hydrate. In instances when withdrawal of these medications is clinically or scientifically indicated, rTMS should be performed, if required, with caution.

(4) There is no literature available yet concerning drugs and the risk of seizure during novel patterned rTMS protocols.

\subsection{State-dependency of rTMS effects}

A growing number of studies indicate that TMS-effectiveness strongly depends on the state of neuronal activation in the targeted brain region at the time of stimulation (see Silvanto and PascualLeone, 2008). Such state-dependent effects are relevant when considering the specificity and focality of TMS, and obviously can potentially lead to relevant safety consideration. Many variables may theoretically contribute, alone or in combination, to change the pre-TMS level of neuronal activity, thereby changing the resulting TMS effects and risks: menstrual cycle (Smith et al., 1999; Inghilleri et al., 2005), age (Rossini et al., 1992, 2007), level of anxiety or mood, sleep deprivation, occult substance abuse, thickness of skull layers or brain atrophy, which may additionally superimpose -in endless combinations- to disease-related variables and concurrent therapies, as stated in previous paragraphs. It probably matters even what subjects and patients do before exposure to TMS/ rTMS, as the effects of the stimulation may be modified.

The basal level of neuronal activity, and in turn of brain reactivity to rTMS, may be further modified both in healthy subjects and patients by transcranial pre-conditioning procedures (i.e., priming) (Iyer et al., 2003; Siebner et al., 2004; Lang et al., 2004), making the resulting effect of rTMS different in term of effect size and even effect direction.

Thus, risks for potential side effects of rTMS applications can theoretically differ between healthy male and female participants and between patients with various diseases, but even across individuals and within individuals over time. There are still no studies specifically addressing the combination of several of the above factors for safety, and further investigations in this sense are warranted. Neurophysiological monitoring for subjects/patients undergoing conventional or patterned rTMS application following not yet explored paradigms of pre-conditioning is desirable.

\subsection{TMS in pediatrics}

Published data from more than 80 studies include reports of TMS applied to about 800 normal and more than 300 neurologically abnormal children, the vast majority (>95\%) receiving either single-pulse or paired-pulse TMS (Frye et al., 2008). Serious adverse events have not been reported in these studies, suggesting that single- and paired-pulse TMS in children is a minimal risk procedure (Gilbert et al., 2004). Yet, TMS safety in pediatrics requires special consideration since developmentally-regulated changes in the CNS may affect susceptibility to TMS-related adverse events. An exhaustive list of developmental changes that may in principle affect TMS safety in children is beyond the practical scope of these guidelines. However, some tangible developmental processes are likely to play a role in pediatric TMS safety: among these are (i) maturation of cortical excitability, (ii) closure of the fontanelle, and (iii) growth of the external auditory canal. We consider these and the relevant pediatric age subgroups as follows:

Maturation of cortical excitability. A characteristic of the neonatal brain is markedly enhanced cortical excitability relative to older children, due to several developmentally-regulated factors. Among these are increased synapse and dendritic spine density, immaturity of intracellular chloride homeostasis leading to depressed inhibition (indeed $\gamma$-aminobutyric acid (GABA) can be an excitatory neurotransmitter in the immature brain), and increased calciumpermeable $\alpha$-amino-3-hydroxyl-5-methyl-4-isoxazole-propionate (AMPA) receptor expression leading to enhanced excitation and potential for excitotoxicity. The immaturity of the inhibition/excitation ratio is evident in the preterm, and peaks before age 1 year. With this increased excitability there is also increased vulnerability to seizure (Silverstein and Jensen, 2007) in the young brain. As a further complication, MT may be higher in the very young children (Frye et al., 2008), and this may equate to increased machine output and greater amplitude electrical activation of the immature brain. Thus, as currently data are lacking, the risk of TMS-induced seizure and potential for excitotoxic injury should be considered especially in the neonate.

(i) Closure of the fontanelle. From birth until approximately 18 months of age, most children continue to have an open fontanelle. This may necessitate special care when placing the coil to avoid mechanical injury. Further, the potential effects of an open fontanelle on the distribution induced electrical field should be considered. 
(ii) Growth of the external auditory canal. Also related to skeletal growth is the relative small caliber and length of the external auditory canal in young children, particularly in neonates. Although the external ear and auditory canal grow throughout childhood, the small external canal volume in early life results in a higher resonance frequency of the external ear from birth until age 2 years when asymptotic values are reached (Kruger, 1987). This theoretically may lead to an increased susceptibility to acoustic injury by high amplitude and high frequency noise. Hence, special care must be taken to protect hearing when applying TMS to children.

Given the available data and the above-mentioned concerns, we cautiously conclude that single-pulse and paired-pulse TMS in pediatrics is safe for children two years and older. For children younger than two years, data about risk for acoustic injury are not available, and therefore specialized hearing protection may be required. Also, for children age one year and younger, safety data are not available, and will have to be obtained. Finally, in absence of an appreciable volume of data on the potential for adverse effects with rTMS, we maintain the previous guideline that children should not be used as subjects for rTMS without compelling clinical reasons, such as the treatment of refractory epilepsy or particular psychiatric conditions.

\subsection{TMS in pregnancy}

Magnetic fields attenuate rapidly with distance, so it seems unlikely that the fetus might be directly affected by TMS. Currently, there are anecdotal reports of pregnant women who underwent successful rTMS treatment for depression, and no side effects to the child were reported (Nahas et al., 1999; Klirova et al., 2008). Nevertheless, a conservative view of the use of rTMS in pregnancy might consider to balancing the risk/benefit ratio for each single case.

For clinical routine use of TMS, direct stimulation on the lumbar spine should be avoided in pregnant women, unless diagnostic compelling reasons are present.

Pregnant women acting as TMS operators should conservatively stay at least $0.7 \mathrm{~m}$ away from the discharging coil (see Section 3.6).

\section{Considerations on dosing TMS}

In addition to the 4 key parameters that define rTMS trains (intensity, frequency, train duration, and inter-train interval), the repeated application of rTMS introduces additional dosing parameters that describe the cumulative exposure to rTMS. These include total pulses per session, sessions per day, days per week, weeks per acute course, and maintenance frequency. Many other emerging variables, besides coil type (see paragraph 1.3), which are addressed in the following paragraphs, should be considered for future safety investigations.

\subsection{Motor threshold (MT), phosphene threshold (PT) and other procedures of stimulation}

The minimal intensity required to elicit an EMG response of at least $50 \mu \mathrm{V}$ with $50 \%$ probability in a fully relaxed muscle (Rossini et al., 1994) is the resting motor threshold (MT). Most of the published studies refer to this classical method for the individualization of the intensity of stimulation (Rossini et al., 1994), even when brain regions outside the motor cortex are targeted, although relationships between the excitability of the motor cortex and other brain regions are not obvious.
Phosphene threshold (PT) is more appropriate to individualize the intensity of stimulation when targeting visual areas, and consists in the minimal intensity required to induce a phosphene in the contralateral visual hemifield (Marg and Rudiak, 1994). PT is a psychophysical measure that does not correlate to MT (Stewart et al., 2001) although a correlation has been described under certain circumstances (Deblieck et al., 2008). It is a common experience that while MT can be easily determined in the vast majority of subjects, the determination of PT may not be obtainable in half of the subjects, and often requires the use of double pulse stimulation (Ray et al., 1998; Boroojerdi et al., 2000).

MT has been performed by visual twitch in some large clinical trials. If precise determination of MT is important, we suggest to avoid this procedure in all future therapeutic studies, since it overestimates the minimal intensity required to activate the motor cortex (due to the lack of monitoring the EMG silence in the target muscle), thereby increasing potential hazards. In addition, it introduces the uncontrolled variable of observer reliability. If MT is measured, it is preferable to use EMG measurements, until future comparative studies definitely determine whether or not a measureable incremental risk actually exists for therapeutic rTMS applications carried out with the visual twitch method. Only $80 \%$ of participants to the consensus meeting questioned the scientific reliability of the visual twitch method, so a full consensus was not reached in regards to this topic.

The relationship between MT, PT and the threshold for TMS activation of other non-motor areas is not known. Nor is it known how illness or concomitant medications may affect that relationship. Therefore, it may be reasonable to consider alternative methods to individualize the intensity of stimulation (e.g., relation to PT or parametric relation to a given index cognitive task). Ultimately, individualized calculations of the induced current density would be desirable, but this is not readily available currently. Realistic head models can provide such measurement. While not all investigators and clinicians may have access to mathematical modeling and computational tools, studies should provide all needed information to allow for eventual calculation of induced currents: coil position, coil orientation, coil geometry and material, current over time, and all available subject data (ideally including brain imaging studies). Knowing current density distribution is a necessary, but not sufficient measure to determine biological, behavioral, and safety effects.

One issue that has been addressed regarding the use of MT to dose non-motor areas is the coil-to-cortex distance. Distance adjustment has been used to compensate for increased coil-to-cortex distance over prefrontal areas, and has been posited to be an important dosing adjustment to adequately dose patients with atrophy (Kozel et al., 2000). This is not an unreasonable procedure with current knowledge. However, the increased distance between coil and brain is filled by tissues of variable conductivity and permittivity, that may alter the current flow and thus more complex modeling of the induced currents, rather than a simple adjustment of TMS intensity for distance may be needed (see Section 2.2 for detailed discussion of these issues).

\subsection{Newer dosing paradigms of rTMS interventions}

Clinical trials in psychiatric patients have typically used low $(1 \mathrm{~Hz})$ or fast $(5-20 \mathrm{~Hz})$ frequencies, however newer dosing paradigms are under investigation including: the sequential application of $5 \mathrm{~Hz}$ followed by $1 \mathrm{~Hz}$ to the same cortical target (priming) (Iyer et al., 2003), the sequential application of high frequency to the left prefrontal cortex followed by low frequency to the right one (Fitzgerald et al., 2006), simultaneous application of high frequency to bilateral prefrontal sites (Loo et al., 2003), TBS (intermittent or continuous), and repetitive paired associative stimulation 
(PAS). Given the clinical interest in examining more complex frequency dosing paradigms, it would be important to characterize the safety profile of such strategies.

Less commonly considered parameters of stimulation concern the characteristics of the pulse itself. rTMS stimulators typically deliver biphasic magnetic waveforms while single-pulse stimulators tend to generate monophasic pulses. These pulses differ in their efficiency in neuronal depolarization. There is recent evidence that monophasic pulses applied repetitively are more efficient in altering cortical excitability (Sommer et al., 2006b; Arai et al., 2005, 2007). This would suggest that safety guidelines would need to be established separately for monophasic rTMS devices. Newly developed controllable pulse TMS (cTMS) circuit topologies have been designed to control other pulse characteristics, including pulse shape (rectangular rather than sinusoidal), pulse width, and degree of bidirectionality (Peterchev et al., 2008). These features are also likely to affect efficiency of depolarization and also potentially side effects.

\subsection{Targets}

The majority of clinical trials have examined single cortical targets. Each target may be expected to have a distinct set of potential side effects given the functional specialization of the cortex and differential trans-synaptic effects. Seizure risk also varies by site. The motor cortex is one of the most excitable brain regions, and, therefore, patterns of stimulation that are safe there are likely to be safe elsewhere. An additional issue is that neurological and psychiatric illnesses are thought to result from distributed networks. A few studies have sought to enhance therapeutic effects by targeting 2 sites, sequentially or simultaneously, with varying degrees of success. The safety of simultaneous multi-site stimulation would need to be carefully examined since this would greatly increase the volume of stimulation and the simultaneous rhythmic stimulation of multiple sites presents epileptogenic potential.

\subsection{Devices}

Devices typically used for therapeutic applications are capable of delivering rTMS with frequencies of $1-20 \mathrm{~Hz}$. Some newer models also provide operator assistive software to facilitate MT determination in the clinical setting. Recently developed devices have an expanded parameter range (in some cases up to $240 \mathrm{~Hz}$ ) to enable TBS and the induction of therapeutic seizures under anesthesia (Magnetic Seizures Therapy), a topic not covered in the present guidelines. The expanded range of these devices introduces the chance of operator error (e.g., accidental administration of a seizure inducing dosage in an unanesthetized subject) and thus careful precaution procedures need to be implemented.

\subsection{Coil navigation systems}

The vast majority of clinical trials targeting the dorsolateral prefrontal cortex in depression have positioned the TMS coil at a scalp location $5 \mathrm{~cm}$ anterior to the optimal site to elicit a motor twitch in a distal hand muscle (often by visual inspection). Many other therapeutic and cognitive applications of rTMS have used similar scalp landmark methods to determine the TMS coil placement. Frameless stereotaxy systems are available to navigate coil position to anatomically or functionally defined targets. These methods have been shown to enhance reliability of placement and the consistency of targeting of a given brain area (Gugino et al., 2001) though the therapeutic value of navigation is just now being explored and has shown some promise in depression (Fitzgerald et al., 2009) and schizophrenia (Hoffman et al., 2007). It is not known how much precision is necessary for therapeutic applications in order to en- sure efficacy and minimize side effects, though Fitzgerald et al (2009) demonstrated improved efficacy with MRI-guided TMS in depression in comparison with conventional methods. There is also a trade-off between practicality/availability of the method and its precision. Other approaches, e.g., using the 10-20 International EEG system, could present a compromise in the clinical setting to inform coil placement.

However, even for the neuroscience research setting, it has been reported recently that enhanced precision of coil placement can improve effect size, thereby decreasing the number of subjects needed (Sack et al., 2009).

\section{Update of the safety guidelines}

The above considerations lead to the development of a new set of guidelines for the safe administration of TMS. The new guidelines, whenever possible, use the 1998 guidelines as a basis. Considerations include ethical and regulatory aspects, stimulation parameters, physiological and neuropsychological monitoring of subjects, settings in which TMS can be done, composition and expertise of the rTMS team, management of potential adverse effects, and contraindications to TMS.

\subsection{Ethical and regulatory issues}

Research and clinical applications of TMS must be governed by three basic ethical and legal requirements pertaining to all studies on human subjects, on which a full consensus has been reached:

(1) Informed consent. The subject's (or legal representative in case of a child or mentally disabled) decision to participate must be voluntary and based on the provision of all relevant information and potential risks. For valid informed consent, subjects need to be provided information in a way they can understand about the procedures, risks and discomforts of the study. Development of standard consent language that describes common TMS procedures in lay terminology and delineates the risks with different types or uses of TMS can assist investigators and help assure Institutional Review Board (IRB) or local Ethic Committees members that these are properly discussed and disclosed.

(2) Risk benefit ratio. The potential benefit of the research must be found by an independent assessment to outweigh the risk. It is not sufficient merely that the subject be willing to accept the risk involved, and there must be no means of obtaining the desired data without placing subjects at risk. In the case of a clinical application, the likelihood of clinical benefit must similarly outweigh the potential risks.

(3) Equal distribution of the burdens and benefits of research. This requirement is violated when research is conducted on categories of patients made vulnerable by economic, social, or physical conditions and who are likely to bear only its burdens.

\subsubsection{Types of rTMS studies}

Permissible rTMS studies may be divided into three classes in the order of their demand for protection of the subjects and expected benefits. Full consensus has been reached on the types of rTMS studies defined herein. In any case, the decision on the risk/benefit ratio of a given rTMS study needs to be made by each principal investigator and her or his IRB or Ethic Committee:

- Class 1 (direct benefit, potential high risk): studies in patients with diagnostic or therapeutic primary objective, including the development of new therapeutic indication or protocols, with potential direct individual clinical benefit. Normal subjects 
should not ordinarily participate in such studies, and the risk level for patients can be theoretically high for stimulation protocols that have been not yet tested for safety.

- Class 2 (indirect benefit, moderate risk): studies in patients where the potential clinical benefit is more speculative or where no clinical benefit is expected, but the study is anticipated to yield valuable data for the development of treatments, safety assessment of a cortical stimulation protocol, or improved understanding of pathophysiological mechanisms of neurological or psychiatric diseases. Normal subjects may participate as control subjects. In these studies, regimens that will place subjects at significant risk of seizures or other serious adverse effects should employ only patients and not normal subjects, because exposure to adverse effects is unacceptable for normal subjects when clinical benefit is questionable.

- Class 3 (indirect benefit, low risk): studies in normal subjects and patients that are expected to yield important data on brain physiology or on safety, but have no immediate relevance to clinical problems. Normal volunteers should be permitted to participate in rTMS research when it is likely to produce data that are of outstanding scientific or clinical value.

In all classes, every appropriate and feasible safety measure must be instituted, and stimulation parameters and schedules must be chosen with clinical goals and safety considerations in mind. Specifically tailored regimens may pose significant risks in some cases, and, indeed, there could be instances where stimulation parameters outside present safety recommendations could be delivered and adverse effects might be expected and prepared for (i.e., Class 1 studies). Nevertheless, the risks should be outweighed by the potential benefit in serious disorders where alternative therapies also have significant risks (e.g., electroconvulsive therapy or other neuromodulatory techniques which requires neurosurgical procedures). In Class 2 and 3 studies the responsibility rests on the Principal Investigator to prove how the participation of normal subjects will enhance the understanding of brain function or advance the understanding or treatment of a disease, in an important way.

Safety studies of new rTMS devices or alternative procedures of TMS must continue to be performed in normal subjects in a manner analogous to toxicity studies of new drugs.

All studies, including safety studies, in normal subjects and patients for whom there is no potential clinical benefit should proceed only with maximally stringent safety measures and limits on stimulation parameters.
The group could not reach consensus about what constitutes a "normal subject". One view is that such persons should have a normal neurologic examination. Another view is that self-reported information is sufficient to establish normalcy. What is appropriate might depend on the investigation. The definition of normalcy should be considered and approved for each study by the referring IRB.

\subsection{Stimulation parameters}

Previous guidelines (Wassermannn, 1998) have proven efficacious in preventing spread of excitation and seizures, both in normal subjects and in patients with neurological and psychiatric diseases, despite the fact that such guidelines were based on a relatively restricted sample of normal subjects and considered only conventional rTMS.

\subsubsection{Conventional rTMS of the motor cortex}

Table 4 is restricted to intensities of stimulation from $90 \%$ to $130 \%$ of resting MT, using a Figure 8 coil. Indeed, intensities higher than $130 \%$ of MT have not been reported for research/clinical purposes, nor have other coils. Future studies eventually exceeding this limit (i.e., from $140 \%$ to $220 \%$ of MT) should refer to previous guidelines (Table 3, Wassermannn, 1998).

In case the individual MT of stimulation cannot be determined due to concomitant drugs, underlying pathology or other anatomo-physiological reason, a consensus emerged for the use of an intensity of stimulation that corresponds to the lower 95\% confidence interval of the average value of the MT in the remaining subjects/population, for the specific coil/stimulator combination.

Another aspect relevant for safety is the length of inter-train intervals in case of repeated applications of rTMS on the same day. To date, no studies have specifically addressed this issue following the original work (Chen et al., 1997). Therefore, we again suggest adopting previous guidelines, at least for motor cortex stimulation, which are summarized in Table 5.

Data of Tables 4 and 5 should provide investigators with the basis for constructing tables with margins of safety appropriate to various types of rTMS studies. Safety margins should be conservative for the protection of subjects in Class 2 and 3 studies. In Class 1 studies, which are of potential direct clinical benefit to the subjects, higher degrees of risk are clearly tolerable, so that stimulation parameters outside these recommendations could be delivered if the benefits are reasonably expected to outweigh the risks. It is also probable that the values in this set of guidelines

\section{Table 5}

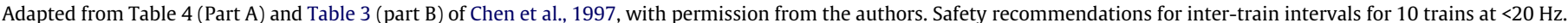

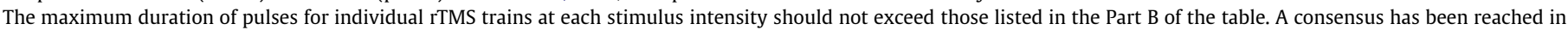
adopting this table at this point. However, there is a need to extend these investigations and provide more detailed guidelines that may apply also to non-motor areas.

\begin{tabular}{|c|c|c|c|c|c|c|c|c|}
\hline \multirow[t]{2}{*}{ Inter-train interval (ms) } & \multicolumn{8}{|c|}{ Stimulus intensity (\% of MT) } \\
\hline & \multicolumn{2}{|c|}{$100 \%$} & & \multicolumn{3}{|c|}{$110 \%$} & \multicolumn{2}{|l|}{$120 \%$} \\
\hline \multicolumn{9}{|l|}{ Part A } \\
\hline 5000 & \multicolumn{2}{|c|}{ Safe } & & \multicolumn{3}{|l|}{ Safe } & \multicolumn{2}{|l|}{ Insufficient data } \\
\hline 1000 & \multicolumn{2}{|c|}{ Unsafe (EMG spread after 3 trains) } & & \multicolumn{3}{|c|}{ Unsafe (EMG spread after 2 trains) } & \multicolumn{2}{|c|}{ Unsafe (EMG spread after 2 trains) } \\
\hline 250 & \multicolumn{2}{|c|}{ Unsafe $^{\mathrm{a}}$} & & \multicolumn{3}{|c|}{ Unsafe (EMG spread after 2 trains) } & \multicolumn{2}{|c|}{ Unsafe (EMG spread after 3 trains } \\
\hline \multirow[t]{2}{*}{ Frequency $(\mathrm{Hz})$} & \multicolumn{2}{|l|}{$100 \%$} & \multicolumn{2}{|l|}{$110 \%$} & \multicolumn{2}{|l|}{$120 \%$} & \multicolumn{2}{|c|}{$130 \%$} \\
\hline & \multicolumn{2}{|c|}{ Duration (s)/pulses } & \multicolumn{2}{|c|}{ Duration (s)/pulses } & \multicolumn{2}{|c|}{ Duration (s)/pulses } & & Duration (s)/pulses \\
\hline \multicolumn{9}{|l|}{ Part B } \\
\hline 1 & $>270$ & $>270$ & $>270$ & $>270$ & $>180$ & $>180$ & 50 & 50 \\
\hline 5 & 10 & 50 & 10 & 50 & 10 & 50 & 10 & 50 \\
\hline 10 & 5 & 50 & 5 & 50 & 3.2 & 32 & 2.2 & 22 \\
\hline 20 & 1.5 & 30 & 1.2 & 24 & 0.8 & 16 & 0.4 & 8 \\
\hline 25 & 1.0 & 25 & 0.7 & 17 & 0.3 & 7 & 0.2 & 5 \\
\hline
\end{tabular}

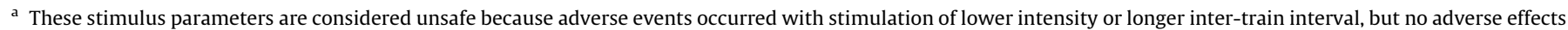
were observed with these parameters. 
may be safely exceeded in subjects receiving anticonvulsant medications, as suggested by a recent study (Rotenberg et al., 2009).

\subsubsection{Conventional rTMS outside the motor cortex}

The above suggested safety parameters are derived from rTMS applied to the motor cortex. Since the threshold for induction of after-discharges is lowest in the motor cortex compared to other cortical areas when stimulated electrically (Penfield and Jasper, 1954), they are reasonably safe also for rTMS applications on cortical areas outside the motor cortex. However, the exact relationships between the excitability of motor and non-motor brain regions are still to be determined. Unfortunately, no studies have specifically addressed this issue, hence definitive safety tables for rTMS application outside the motor cortex cannot be provided. There are still no studies addressing the safety of rTMS as a function of the threshold to evoke phosphenes.

Nevertheless, certain combinations of parameters of stimulation that have been applied outside the motor cortex without serious adverse effects can be found in two recent reviews (Machii et al., 2006; Loo et al., 2008). Thus, the accumulated experience in the past 20 years provides a strong substrate that investigators can use to inform arguments about the safety and relative risk of rTMS protocols.

\subsubsection{Patterned repetitive TMS}

TBS protocols are increasingly used, both in research and for clinical applications. There is only one study specifically addressing the safety of TBS in 24 healthy subjects who received stimulation on left dorsolateral and medial prefrontal cortices (Grossheinrich et al., 2009): no serious adverse effects were noted, apart from lipotimiclike reactions in three subjects. However, due to the paucity of safety studies on TBS, safety guidelines cannot be currently provided.

Table 6 summarizes TBS protocols used up to now in published studies on normal subjects. As described above, a single seizure has been reported, induced by cTBS in a normal volunteer (Obermann and Pascual-Leone, 2009). In that instance cTBS was applied at an intensity of $90 \%$ of resting motor threshold which might translate to an intensity of approximately $120 \%$ of active motor threshold, thus much higher than most published trials of cTBS. Table 6 also summarizes available studies using QPS paradigms. It is important to note that the safety of these protocols in patients with neurological or psychiatric brain diseases, or when applied under pharmacological treatments or outside the motor cortex, is still to be determined.

Several aspects still need to be evaluated in terms of safety, even in normal subjects:

(i) Total pulse number: traditional TBS protocols pose the current limit at 600 , but 900 has been safely performed (J. Rothwell, personal communication); (ii) Interval between repeated TBS sessions: 15 min being safe in 6 normal subjects (Nyffeler et al., 2006);

(iii) Intensity of stimulation: the maximal intensity safely used so far being $60 \%$ of the maximal stimulator output over the visual cortex (Silvanto et al., 2007) and $80 \%$ of the resting MT for prefrontal cortex stimulation (Grossheinrich et al., 2009);

(iv) Cumulative daily or weekly applications for therapeutic purposes.

\subsubsection{Physiological monitoring}

Physiological monitoring of every subject undergoing conventional or patterned repetitive TMS is desirable when parameters of stimulation exceed previous tables. This applies to studies of Classes 1, 2 and 3.

Two measures have been proposed to detect potential early signs of increasing brain excitability that might lead to a seizure: spread of excitation to neighboring cortical areas and possible manifestations of EEG afterdischarges.

In studies where rTMS is not expected to elicit MEPs (e.g., stimulation of the motor cortex below threshold, or of a scalp site outside it at any intensity), the EMG can be monitored continuously from a hand muscle, such as the abductor pollicis brevis or the first dorsal interosseous muscle, on the side contralateral to rTMS. These muscles have the lowest threshold for the production of MEPs, and the appearance of MEPs during an experiment may indicate the spread of excitation from neighboring areas to the motor cortex.

In studies where the stimulation is expected to produce MEPs in a distal muscle (i.e., the hand), an additional muscle at a proximal segment of the same limb can be monitored. The appearance of "proximal" MEPs in a forearm muscle (such as the extensor carpi radialis) or in an arm muscle (as the deltoid), would indicate the intra-cortical spread of excitation or lowering of the MT.

Visual monitoring of subjects during rTMS is mandatory. Muscle twitching time-locked to the stimulus provide a potentially important indication of spread of evoked motor activity, albeit less sensitive than EMG monitoring (Lorenzano et al., 2002). It might be advisable to use video monitoring in high-risk studies. Subjects should be observed by a qualified individual at all times during rTMS.

Theoretically, the EEG would seem the most appropriate tool for physiological monitoring of rTMS effects, since afterdischarges following the cessation of cortical stimulation are traditionally considered the first indicator of induced epileptic activity (Ajmone-Marsan, 1972), and are routinely used as a safety feature in subdural cortical stimulation and recording. However, routine EEG monitoring in normals and patient populations is unlikely to increase safety of TMS significantly, given the low incidence of

Table 6

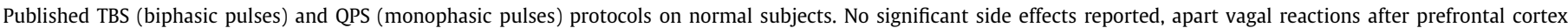
stimulation. Consensus reached for this table.

\begin{tabular}{|c|c|c|c|c|}
\hline & Pulses in the burst & Total train pulses & Intensity & Stimulation site \\
\hline "Standard" cTBS (following Huang et al. 2005) & 3 at $50 \mathrm{~Hz}$, repeated at $5 \mathrm{~Hz}$ & $600(40 s)$ & $80 \%$ of active MT & Motor cortex, $\mathrm{PFC}^{\mathrm{C}}$ \\
\hline Silvanto et al. 2007 & 8 at $40 \mathrm{~Hz}$, repeated every $1.8 \mathrm{~s}$ & 200 & $\begin{array}{l}60 \% \text { of the maximal } \\
\text { stimulator output }\end{array}$ & Visual cortex \\
\hline Nyffeler et al. $2006^{a}$ & 3 at $30 \mathrm{~Hz}$, repeated at $10 \mathrm{~Hz}$ & 200 & $80 \%$ of resting MT & Frontal eye fields \\
\hline $\begin{array}{l}\text { "Standard" iTBS protocols (following } \\
\text { Huang et al. 2005) }\end{array}$ & 3 at $50 \mathrm{~Hz}$, repeated at $5 \mathrm{~Hz}$ for $2 \mathrm{~s}$ & 600 & $80 \%$ of active MT & Motor cortex, $\mathrm{PFC}^{\mathrm{C}}$ \\
\hline QPS $^{\text {b }}$ (following Hamda et al., 2008) & $\begin{array}{l}4 \text { (ISI ranging } 1.5 \mathrm{~ms}-1.25 \mathrm{~s} \text { ), } \\
\text { repeated every } 5 \mathrm{~s}\end{array}$ & 1440 & $90 \%$ of active MT & Motor cortex \\
\hline
\end{tabular}

\footnotetext{
a Also repeated TBS in the same session (at 5, 15, 60, $75 \mathrm{~min}$ ).

b 2000 maximal total pulse number per day; highest intensity used resting MT (Y. Ugawa, personal communication).

c $\mathrm{PFC}=$ prefrontal cortex (Grossheinrich et al. 2009).
} 
TMS-induced epileptiform activity and the low predictive value of epileptiform discharges for seizure induction (see Sections 4.2 and Section 4.3). Nonetheless, future safety studies will probably benefit from co-registration of TMS and EEG activity, once this technology becomes more widely available in clinical settings.

Consensus has been reached on the suggested modalities of physiological monitoring.

\subsubsection{Neuropsychological monitoring}

Long-term cognitive and neuropsychological changes of single rTMS applications seem negligible (see Table 1, Sections 4.6 and 4.7).

Neuropsychological monitoring is still strongly recommended when cumulative daily sessions of rTMS are administered for therapeutic purposes (Class 1 studies) or, for Class 2 and 3 studies, when new parameters of stimulation are investigated. This applies particularly to patterned rTMS protocols, whose neuropsychological sequelae are still scarcely investigated.

Besides subjects' self reports, objective evaluations are required in these studies: these should be short and easy to administer, but sensitive enough to detect subtle deficits. Cognitive test batteries may change according to the stimulated region and to the expected effects. It is responsibility of the principal investigator to decide the most appropriate cognitive battery to be applied, but, of course, there should be approval by the IRB.

Consensus has been reached on the suggested modalities of neuropsychological monitoring.

\subsubsection{Where should TMS be done}

The increasing use of TMS makes it necessary to consider places where TMS can be carried out safely, taking into consideration both research and clinical needs. For diagnostic and therapeutic applications of TMS in patients (Class 1 and Class 2 studies) a medical setting is required. For Class 3 studies and Class 2 studies on normal subjects, carried out with TMS or rTMS parameters not exceeding those of Tables 4-6, conduct of the study in a medical setting may not be required. The referring IRB is the final arbiter for such considerations.

For all TMS and rTMS research studies, approval from the Institutional Review Board and a signed informed consent from the study participants should be obtained. The Principal Investigator of a TMS or rTMS study does not need to be a licensed physician, but should be an expert in TMS with knowledge about principles, physiology and potential side effects of the technique. In addition, appropriate emergency medical attention for possible TMS complications should be planned for. A licensed physician that is intimately familiar with the study protocol, the risks of TMS, the treatment of any of its possible complications and side effects, and the condition of any patients undergoing TMS, should be involved in the design and conduct of study protocols. Therefore, TMS and rTMS protocols should identify a principal investigator and a medically responsible physician.

Possible uses and settings of TMS, based on what is more extensively discussed in previous paragraphs, are summarized schematically in Table 7. It is clear that the risk of a given TMS study has to take into consideration all aspects of the study, not simply the TMS parameters. The responsibility to assess the risk of a TMS study, like that of any study, lies with the Pincipal Investigator who has to obtain the appropriate approval from his/her IRB.

\subsubsection{Hospital, outpatient setting or research labs?}

To date, research with single-, paired-pulse and conventional rTMS has been carried out safely in many laboratories outside of medical setting across the world (i.e., psychology, physiology, robotics). Despite it being very low, the risk of an adverse effect is, however, not completely absent. Therefore, the Principal Inves- tigator of the study has to balance this possibility, plan for possible complications, and fully comply with the local IRB rules.

A medical setting (hospital or appropriately equipped outpatient clinic) is needed for all clinical applications of TMS (i.e., diagnostic or interventional procedures of neuromodulation). Outpatient TMS treatments can be delivered outside of a hospital. However, it is strongly advisable that in these settings and in other medical environments, appropriate life-support equipment and emergency medical facilities be available.

All medical applications of TMS should be done under the supervision of a responsible physician, who is responsible for handling all adverse events and complications. Whether the responsible physician has to be present in the laboratory at the time of TMS/rTMS application or not, depends on the circumstances and is a decision to be made by the study's Principal Investigator, and the responsible physician in collaboration with their Iocal IRB, Ethics Board, or Medical Board.

\subsubsection{TMS in special neuroimaging environments}

Despite the widespread diagnostic and neuroscientific use and the increasing therapeutic application, TMS-brain interactions still need to be fully elucidated. This motivates the combination of TMS with neuroimaging techniques, such as single photon positron emission computed tomography (SPECT), positron emission tomography (PET), functional magnetic resonance imaging (fMRI) or EEG (see paragraph 3.2). The combined neuroimaging-TMS approach has been used to localize the neural structures that are actually targeted by TMS, characterize the impact of TMS on regional brain function, assess the duration of these effects, and determine the functional state of the stimulated region, which might affect the behavioural response (Siebner et al., 2009). Assessing the safety of TMS with neuroimaging becomes increasingly relevant in light of current attempts that seek to increase the efficacy of TMS as a means to induce lasting changes in brain function.

To date, more than 40 combined TMS-PET or TMS-SPECT, and more than 30 TMS-fMRI studies have been reported, including more than 500 participants (see Bestmann et al., 2008 and Siebner et al., 2008, for recent overviews). In some studies, TMS was given during neuroimaging (i.e., "online" approach), while in other studies TMS and neuroimaging were separated in (space and) time (i.e., "offline" approach). No adverse effects, different from those encountered in other TMS studies, and no special long-term adverse effects have been reported in these studies even though a wide range of TMS protocols with up to 1800 pulses (PET) or 960 pulses (fMRI) have been applied.

When TMS is applied before or after neuroimaging, TMS is usually given outside the scanner room. Here the main goal is to examine the lasting effects of TMS on human brain function. This offline approach is technically straightforward and does not result in additional safety concerns, as long as the stimulation is performed outside the scanner room (for TMS in the MR scanner see below). Of course, the general health and safety concerns apply as for neuroimaging studies without TMS. Monitoring of the subject vital signs should be carried out (e.g., blood pressure, heart rate, etc.) particularly if subjects stay alone in the scanner room, for instance during injection of the radioisotope and PET scanning.

Online TMS-neuroimaging studies are performed to probe acute changes in human brain function at the time of TMS administration. The online approach is technically more challenging, especially when TMS is given in the MRI environment. While online TMS during PET or SPECT poses no particular safety problems, if TMS is delivered in the MR scanner, there are potential safety concerns which are related to the static magnetic field of the MR scanner, the RF pulses and gradients applied during scanning, and the mechanic interactions between the TMS system and static magnetic field of the scanner. 
Table 7

Possible uses and settings of TMS

\begin{tabular}{|c|c|c|c|c|c|}
\hline Use & Single-pulse & $\begin{array}{l}\text { Paired- } \\
\text { pulse, } \\
\text { rTMS } \leqslant 1 \mathrm{~Hz}\end{array}$ & $\begin{array}{l}\text { Conventional } \\
\text { rTMS, }>1 \mathrm{~Hz}\end{array}$ & $\begin{array}{l}\text { Patterned rTMS } \\
\text { (TBS, QPS) }\end{array}$ & $\begin{array}{l}\text { Conventional or patterned rTMS with } \\
\text { parameters exceeding those of Tables 3-5 }\end{array}$ \\
\hline $\begin{array}{l}\text { Research (Class } 3 \text { studies; Class } 2 \text { studies } \\
\text { in normal subjects) }\end{array}$ & $\square$ & $\square$ & $\square$ & $\square 0$ & $\boldsymbol{\square} \bigcirc$ \\
\hline Diagnostic applications & $\square$ & $\square$ & $\mathbf{0} \bullet$ & Currently not done & Currently not done \\
\hline $\begin{array}{l}\text { Therapy/neuromodulation (Class } 1 \text { studies and } \\
\text { Class } 2 \text { studies in patients) }\end{array}$ & Currently not done & $\mathbf{\square}$ & $\mathbf{\square} \bullet$ & $\boldsymbol{\square} \bullet$ & - \\
\hline
\end{tabular}

$\square=$ Non medical setting allowable (i.e., psychology labs, robotics labs, research institutions, etc.). Presence of a Physician in the premises may not be required.

- Medical setting required

- = A medically responsible physician is required. Personnel skilled in the management of syncope and seizure is required in the lab.

$\mathrm{O}=$ Emergency medical assistance is strongly recommended. Personnel skilled in the management of syncope and seizure should be present in the lab.

The major issues that need to be taken into consideration when performing TMS in the MRI environment include:

1. All ferromagnetic material must be removed from the stimulation coil and all other parts and devices possibly coming close to the scanner. Otherwise, strong forces and torques will act on the items introduced into the scanner.

2. If ferromagnetic TMS equipment is taken into the scanner room, it needs to be adequately anchored and secured. In addition, the cabling of the TMS coil may provide additional concerns, as it may lead to tripping. This should be avoided by appropriately securing the TMS cabling.

3. Only dedicated TMS coils that have been approved for MR environment must be used. The safety of TMS coils needs to be certified by the manufacturer taking into account the field strength of the MR scanner. Their mechanical integrity must be checked on a recommended basis, to ensure proper functioning despite wear and tear. At present, no experience exists with round TMS coils, multiple TMS coils, or specialized TMS coils; most TMS studies in the scanner have used Figure 8 coils. Coil MRcompatibility needs to be assessed prior to scanning, and further assessed during MR scanning with a phantom prior to scanning on a human head.

4. TMS coils are generally heavy and they need to be positioned over the subjects scalp. In the MR environment, the pulsing MR magnetic field can induce torque forces in the coil which could make it move and pose a mechanical threat to the subject. Therefore, any movements of the coil should be avoided, e.g., by use of MR-compatible TMS coil holders.

5. At present, studies have been conducted at field strengths of $1.5,2$ and $3 \mathrm{~T}$. The interaction of the static magnetic field of the MR scanner and the induced magnetic field lead to mechanical stress of the TMS coil, which is likely to increase at higher field strength. No data exists for $>3 \mathrm{~T}$, and careful tests will have to be carried out in such cases, to ensure the mechanical stability of the TMS coil.

6. The mechanical stress is likely to increase with increasing field strength, resulting in strong auditory stimulation. Subjects should always wear earplugs, plus MR-compatible dedicated head phones.

7. Participants should always be monitored during scanning (e.g., using pulse oxymetry), as is the case often already for fMRI studies $\geqslant 3 \mathrm{~T}$.

8. Safety of MR head coils needs to be ensured. For example, TMS pulses must not be fired directly on any electronic circuits within the MR head coil.

\subsubsection{Managing emergencies (syncope and seizures)}

Each TMS laboratory must institute an explicit plan for dealing with syncope and seizures, and every member of the TMS team must be familiar with it. There must be a place where the subject can lie down. All team members must be familiar with the means of summoning emergency medical help and when to call for it.
Additionally, in laboratories performing rTMS at $>1 \mathrm{~Hz}$, life-support equipment should be available.

Syncope usually is very brief. Seizures potentially induced by TMS, as seizures in general, are also brief (typically $<60 \mathrm{~s}$ ) and without serious physical sequelae. Thus, efforts should be focused on preventing complications of the seizure or syncope rather than starting any specific medication, which is not required unless status epilepticus (which has been never described following rTMS) occurs. In most cases it is enough to lay the subject down. Supine position with the legs elevated is appropriate for suspected syncope. In case of seizures, attention must be taken to minimize the risk of aspiration and left lateral decubitus position is desirable.

The description of additional medical emergency procedures to treat seizure complications go beyond the scope of the current guidelines.

Subjects who experience seizures with rTMS should be informed of the fact that they are not at a greater risk for further seizures than before. For some individuals, however, the potential psychological effects of having had a seizure can be significant and should not be ignored or minimized. Informed consent documents should clearly discuss the possibility of a seizure, and investigators must ensure that the subjects understand its implications. Both medical and psychological support must be provided to patients and normal subjects who have rTMS-induced seizures.

It is readily imaginable that the report of a seizure in the medical record of a normal volunteer or certain patients could be misinterpreted or deliberately used as a pretext for the denial of employment or medical insurance. Subjects of research studies must be informed of this possibility, and investigators must make certain that documentation of seizures is done in such a way that jeopardizes subjects to the minimum extent possible. Additional documentary support of a healthy subject's claim that a provoked seizure carries no adverse prognosis must be provided when appropriate.

\subsection{Who should do TMS}

This document does not pretend to provide an in-depth discussion of the training and accreditation requirements to conduct TMS/rTMS studies or apply it in medical settings. In fact, such requirements may vary extensively from country to country. The following paragraphs simply raise some fundamental aspects aimed at promoting the safety of the procedure. Consensus has been reached on the following points regarding credentials and team as well as training.

\subsubsection{Credentials and team}

Required credentials for the application of TMS depend on the type of TMS study (Classes 1,2 and 3) and by the purpose and parameters of the TMS application:

(a) Clinical routine use of TMS for investigation of motor tracts, prescribed by a licensed physician, can be carried out by properly 
trained technicians. A neurologist or clinical neurophysiologist should supervise any investigation and provide a written medical report. The risk of adverse effects is very small.

(b) Research studies (Class 3 studies and Class 2 studies) with single-pulse, paired-pulse TMS, rTMS at $\leqslant 1 \mathrm{~Hz}$ and other conventional or patterned rTMS which fall within known safety margins (see Tables 3-6) on normal subjects and patients with stable medical conditions can be carried out by trained professionals (MDs, Technicians, Psychologists, Physicists, Physiotherapists, Engineers), under the responsibility of the Principal Investigator, whose physical presence in the lab is not required, but who should be immediately available. $\mathrm{He} / \mathrm{she}$ is also responsible of the training of TMS users. Medical assistance is strongly recommended for Class 2 studies on patients, for which personnel skilled in syncope and seizure management should be present in the lab. In these cases, a licensed physician should be identified as the medically responsible clinician and should oversee screening procedures, including assessment of risk factors, rTMS parameters and application protocol, and monitoring of subjects.

(c) When rTMS is prescribed (by an MD) as treatment for any medical condition (Class 1 studies), it is advisable that a licensed physician, serving as medically responsible clinician, closely supervises the rTMS application given the more likely medical instability of the patients. The rTMS application can be carried out by a properly trained medical assistant. All personnel have to be trained to recognize and to manage a seizure or a syncope, and there should be full access to emergency treatment and life-support equipment. The responsible clinician should also make sure that the medical assistant is properly trained in how to deal with potential acute complications.

Whenever monitoring is required by the type of study (see Section 7.2.4), the research team should always include a qualified clinical neurophysiologist to supervise the recording and interpretation of electrophysiological data. A physician, a nurse or other qualified personnel who has experience with rTMS and is skilled in the management of first aid for seizures should be present in the rTMS laboratory in these cases.

\subsubsection{Training}

To date, there is no official position about training requirements. It is however advisable that every TMS user, especially if he/she lacks medical training, has basic knowledge of brain physiology, of basic mechanisms of TMS, of the potential risks of the procedure, of the physiological changes induced, etc. The Principal Investigator of the study is responsible for guaranteeing the proper training of TMS operators working with him/her. Such training should also include the ability and certification to deal with potential acute complications of TMS. Training may vary according to the TMS use.

Teaching courses are not mandatory at the moment, but there are some offered in different countries and organized by public or private institutions, as well as by some national Societies of Clinical Neurophysiology. It is clear that training requirements will need to be consensual, and that different national guidelines may eventually need to be developed. The IFCN has commissioned a forthcoming paper on training requirements for TMS use.

\subsection{Contraindications and precautions}

The bulk of TMS studies over the last decade following the 1998 published guidelines suggest that the following considerations can be made, for which full consensus was reached:
1. The only absolute contraindication to TMS/rTMS is the presence of metallic hardware in close contact to the discharging coil (such as cochlear implants, or an Internal Pulse Generator or medication pumps). In such instances there is a risk of inducing malfunctioning of such implanted devices.

2. Conditions of increased or uncertain risk of inducing epileptic seizure are:

a. Related to the protocol of stimulation:

i. Any "novel paradigm" (i.e., that is not a classical method of high-/low-frequency rTMS, performed with a flat Figure 8 coil and biphasic pulse waveform). Pre-conditioning (i.e., priming), TMS applied on more than a single scalp region, and prolonged PAS protocols are included in this category.

ii. Conventional high-frequency rTMS protocol with parameters of stimulation (intensity, frequency, train length or intertrain duration) exceeding the known safety limits reported in the Tables 4-6 of Section 7.2.

b. Related to the disease or patient's condition:

i. Personal history of epilepsy (untreated patients with one or a few past episodes), or treated patients.

ii. Vascular, traumatic, tumoral, infectious, or metabolic lesion of the brain, even without history of seizure, and without anticonvulsant medication

iii. Administration of drugs that potentially lower seizure threshold (see Section 5.3 for a full list), without concomitant administration of anticonvulsant drugs which potentially protect against seizures occurrence

iv. Sleep deprivation, alcoholism

3. Conditions of increased or uncertain risk of other events are:

c. Related to patient's condition:

i. Implanted brain electrodes (cortical or deep-brain electrodes) (see Section 3.4)

ii. Pregnancy

iii. Severe or recent heart disease

4. No risk: none of the previous conditions and single- or pairedpulse TMS or conventional low- or high-frequency rTMS protocol with parameters of stimulation (intensity, frequency, train length or intertrain duration) within the "safety limits" reported in the Tables 4-6 of Section 7.2.

\subsection{A screening standard questionnaire for rTMS candidates}

Investigators should consider using a standard questionnaire to screen rTMS candidates. The following questions represent the basic information required. Additional information may change according to particular demands. Consensus has been reached for this questionnaire.

1. Do you have epilepsy or have you ever had a convulsion or a seizure?

2. Have you ever had a fainting spell or syncope? If yes, please describe in which occasion(s)

3. Have you ever had severe (i.e., followed by loss of consciousness) head trauma?

4. Do you have any hearing problems or ringing in your ears?

5. Are you pregnant or is there any chance that you might be?

6. Do you have metal in the brain/skull (except titanium)? (e.g., splinters, fragments, clips, etc.)

7. Do you have cochlear implants?

8. Do you have an implanted neurostimulator? (e.g., DBS, epidural/subdural, VNS) 
9. Do you have a cardiac pacemaker or intracardiac lines or metal in your body?

10. Do you have a medication infusion device?

11. Are you taking any medications? (Please list)

12. Did you ever have a surgical procedures to your spinal cord?

13. Do you have spinal or ventricular derivations?

14. Did you ever undergo TMS in the past?

15. Did you ever undergo MRI in the past?

Affirmative answers to one or more of questions 1-13 do not represent absolute contraindications to TMS, but the risk/benefit ratio should be carefully balanced by the Principal Investigator of the research project or by the responsible (treating) physician.

\section{Acknowledgements}

The workshop was supported by the International Federation of Clinical Neurophysiology (IFCN), the European Chapter of the IFCN, the National Institute of Neurological Disorders and Stroke, the University of Siena, as well as unrestricted gifts from Magstim, Nexstim, and Neuronetics. APL was also supported by the Berenson-Allen Family Foundation.

Authors thank Matteo Feurra, Psy. D. for providing Fig. 2.

\section{Appendix A. Supplementary data}

Supplementary data associated with this article can be found, in the online version, at doi:10.1016/j.clinph.2009.08.016.

\section{References}

Abraham WC, Bear MF. Metaplasticity: the plasticity of synaptic plasticity. Trends Neurosci 1996;19:126-30.

Adams RD, Victor M. Faintness and Syncope. In: Principles of Neurology. 2nd ed. New York: McGraw Hill; 1977. p. 248-57 [Chapter 17].

Ajmone-Marsan C. Focal electrical stimulation. In: Purpura DP, Penry JK, Tower DB, Woodbury DM, Walter RD, editors. Experimental Models of Epilepsy. New York: Raven Press; 1972. p. 147-72.

Alagona G, Coco M, Rapisarda G, Costanzo E, Maci T, Restivo D, et al. Changes of blood lactate levels after repetitive transcranial magnetic stimulation. Neurosci Lett 2009;450:111-3.

Aleman A, Sommer IE, Kahn RS. Efficacy of slow repetitive transcranial magnetic stimulation in the treatment of resistant auditory hallucinations in schizophrenia: a meta-analysis. J Clin Psychiat 2007;68:416-21.

Amassian VE, Eberle L, Maccabee PJ, Cracco RQ. Modelling magnetic coil excitation of human cerebral cortex with a peripheral nerve immersed in a brain-shaped volume conductor: the significance of fiber bending in excitation. Electroencephalogr Clin Neurophysiol 1992;85:291-301.

Anderson B, Mishory A, Nahas Z, Borckardt JJ, Yamanaka K, Rastogi K, et al. Tolerability and safety of high daily doses of repetitive transcranial magnetic stimulation in healthy young men. J ECT 2006;22:49-53.

Anderson BS, Kavanagh K, Borckardt JJ, Nahas ZH, Kose S, Lisanby SH, et al. Decreasing procedural pain over time of left prefrontal rTMS for depression: initial results from the open-label phase of a multi-site trial (OPT-TMS). Brain Stimul 2009;2:88-92.

Arai N, Okabe S, Furubayashi T, Terao Y, Yuasa K, Ugawa Y. Comparison between short train, monophasic and biphasic repetitive transcranial magnetic stimulation (rTMS) of the human motor cortex. Clin Neurophysiol 2005;116:605-13.

Arai N, Okabe S, Furubayashi T, Mochizuki H, Iwata NK, Hanajima R, et al. Differences in after-effect between monophasic and biphasic high-frequency rTMS of the human motor cortex. Clin Neurophysiol 2007;118:2227-33.

Arana AB, Bockardt JJ, Ricci R, Anderson B, Li X, Linder KJ, et al. Focal electrical stimulation as a Sham control for rTMS: does it truly mimic the cutaneous sensation and pain of active prefrontal rTMS? Brain Stimul 2008;1:44-51.

Bae EH, Schrader LM, Machii K, Alonso-Alonso M, Riviello Jr JJ, Pascual-Leone A, et al. Safety and tolerability of repetitive transcranial magnetic stimulation in patients with epilepsy: a review of the literature. Epilepsy Behav 2007;10:521-8.

Barker AT. An introduction to the basic principles of magnetic nerve stimulation. J Clin Neurophysiol 1991;8:26-37.

Basser PJ, Roth BJ. Stimulation of a myelinated nerve axon by electromagnetic induction. Med Biol Eng Comput 1991;29:261-8.

Bear MF. Bidirectional synaptic plasticity: from theory to reality. Philos Trans R Soc Lond B Biol Sci 2003;358:649-55.
Bernabeu M, Orient F, Tormos JM, Pascual-Leone A. Seizure induced by fast repetitive transcranial magnetic stimulation. Clin Neurophysiol 2004;115:1714-5.

Bestmann S, Ruff CC, Blankenburg F, Weiskopf N, Driver J, Rothwell JC. Mapping causal interregional influences with concurrent TMS-fMRI. Exp Brain Res 2008;191:383-402.

Bockart JJ, Smith AR, Hutcheson K, Johnson K, Nahas Z, Andrson B, et al. Reducing pain and unpleasantness during repetitive transcranial magnetic stimulation. J ECT 2008;22:259-64.

Bonato C, Miniussi C, Rossini PM. Transcranial magnetic stimulation and cortical evoked potentials: a TMS/EEG co-registration study. Clin Neurophysiol 2006;117:1699-707.

Boroojerdi B, Bushara KO, Corwell B, Immisch I, Battaglia F, Muellbacher W, et al. Enhanced excitability of the human visual cortex induced by short-term light deprivation. Cer Cortex 2000;10:529-34.

Boutros NN, Berman RM, Hoffman R, Miano AP, Campbell D, Ilmoniemi R Electroencephalogram and repetitive transcranial magnetic stimulation. Depress Anxiety 2000;12:166-9.

Boutros NN, Miano AP, Hoffman RE, Berman RM. EEG monitoring in depressed patients undergoing repetitive transcranial magnetic stimulation. J Neuropsychiat Clin Neurosci 2001;13:197-205.

Bradley JK, Nyekiova M, Price DL, Lopez LD, Crawley T. Occupational exposure to static and time-varying gradient magnetic fields in MR units. J Magn Reson Imag 2007;26:1204-9.

Branston NM, Tofts PS. Analysis of the distribution of currents induced by a changing magnetic field in a volume conductor. Phys Med Biol 1991;36:161-8.

Bridgers SL, Delaney RC. Transcranial magnetic stimulation: an assessment of cognitive and other cerebral effects. Neurology 1989;39:417-9.

Brighina F, Piazza A, Vitello G, Aloisio A, Palermo A, Daniele O, et al. RTMS of the prefrontal cortex in the treatment of chronic migraine: a pilot study. J Neurol Sci 2004;227:67-71.

Brix G, Seebass M, Hellwig G, Griebel J. Estimation of heat transfer and temperature rise in partial-body regions during MR procedures: an analytical approach with respect to safety considerations. Magn Reson Imag 2002;20:65-76.

Cantello R, Rossi S, Varrasi C, Ulivelli M, Civardi C, Bartalini S, et al. Slow repetitive TMS for drug-resistant epilepsy: clinical and EEG findings of a placebocontrolled trial. Epilepsia 2007;48:366-74.

Cappa SF, Sandrini M, Rossini PM, Sosta K, Miniussi C. The role of the left frontal lobe in action naming: rTMS evidence. Neurology 2002;59:720-3.

Chen R, Gerloff C, Classen J, Wassermannn EM, Hallett M, Cohen LG. Safety of different inter-train intervals for repetitive transcranial magnetic stimulation and recommendations for safe ranges of stimulation parameters. Electroencephalogr Clin Neurophysiol 1997;105:415-21.

Chen R, Yung D, Li JY. Organization of ipsilateral excitatory and inhibitory pathways in the human motor cortex. J Neurophysiol 2003;89:1256-64.

Clow A, Lambert S, Evans P, Hucklebridge F, Higuchi K. An investigation into asymmetrical cortical regulation of salivary S-IgA in conscious man using transcranial magnetic stimulation. Int J Psychophysiol 2003;47:57-64.

Collado-Corona MA, Mora-Magaña I, Cordero GL, Toral-Martiñón R, ShkurovichZaslavsky M, Ruiz-Garcia M, et al. Transcranial magnetic stimulation and acoustic trauma or hearing loss in children. Neurol Res 2001;23:343-6.

Conca A, Konig P, Hausmann A. Transcranial magnetic stimulation induces 'pseudoabsence seizure'. Acta Psychiat Scand 2000;101:246-8.

Conte A, Gilio F, Iacovelli E, Bettolo CM, Di Bonaventura C. Frasca V, et al. Effects of repetitive transcranial magnetic stimulation on spike-and-wave discharges. Neurosci Res 2007;57:140-2.

Corthout E, Barker AT, Cowey A. Transcranial magnetic stimulation. Which part of the current waveform causes the stimulation? Exp Brain Res 2001;141:128-32.

Counter SA. Neurobiological effects of extensive transcranial electromagnetic stimulation in an animal model. Electroencephalogr Clin Neurophysio 1993:89:341-8.

Counter SA, Borg E. Analysis of the coil generated impulse noise in extracranial magnetic stimulation. Electroencephalogr Clin Neurophysiol 1992;85:280-8.

Couturier JL. Efficacy of rapid-rate repetitive transcranial magnetic stimulation in the treatment of depression: a systematic review and meta-analysis. J Psychiat Neurosci 2005;30:83-90.

Davey KR, Epstein CM. Magnetic stimulation coil and circuit design. IEEE Trans Biomed Eng 2000;47:1493-9.

Davey KR, Riehl ME. Suppressing the surface field during transcranial magnetic stimulation. IEEE Trans Biomed Eng 2006;53:190-4.

De Lucia M, Parker GJM, Embleton K, Newton JM, Walsh V. Diffusion tensor MRIbased estimation of the influence of brain tissue anisotropy on the effects of transcranial magnetic stimulation. NeuroImage 2007;36:1159-70.

Deblieck C, Thompson B, Iacoboni M, Wu AD. Correlation between motor and phosphene thresholds: a transcranial magnetic stimulation study. Hum Brain Mapp 2008;6:662-70.

Deng ZD, Peterchev A, Lisanby SH. Coil design considerations for deep-brain transcranial magnetic stimulation (dTMS). Proc IEEE Eng Med Biol Soc 2008:5675-9.

Devlin JT, Watkins KE. Stimulating language: insights from TMS. Brain 2007;130:610-22.

Dhuna A, Gates J, Pascual-Leone A. Transcranial magnetic stimulation in patients with epilepsy. Neurology 1991;41:1067-71.

Di Lazzaro V, Thickbroom GW, Pilato F, Profice P, Dileone M, Mazzone P, et al. Direct demonstration of the effects of repetitive paired-pulse transcranial magnetic stimulation at I-wave periodicity. Clin Neurophysiol 2007;118:1193-7. 
Di Lazzaro V, Pilato F, Dileone M, Profice P, Oliviero A, Mazzone P, et al. The physiological basis of the effects of intermittent theta burst stimulation of the human motor cortex. J Physiol 2008;586:4481-7.

Di Lazzaro V, Dileone M, Pilato F, Profice P, Cioni B, Meglio M et al. Long-term motor cortex stimulation for amyotrophic lateral sclerosis. Brain Stimulation, 2009 [epub ahead of print].

Dräger B, Breitenstein C, Helmke U, Kamping S, Knecht S. Specific and nonspecific effects of transcranial magnetic stimulation on picture-word verification. Eur J Neurosci 2004;20:1681-7.

Duning T, Rogalewski A, Steinstraeter O, Kugel H, Jansen A, Breitenstein C, et al. Repetitive TMS temporarily alters brain diffusion. Neurology 2004;62:2144.

Dwork AJ, Arango V, Underwood M, Ilievski B, Rosoklija G, Sackeim HA, et al. Absence of histological lesions in primate models of ECT and magnetic seizure therapy. Am J Psychiat 2004;161:576-8.

Eaton $\mathrm{H}$. Electric field induced in a spherical volume conductor from arbitrary coils: application to magnetic stimulation and MEG. Med Biol Eng Comput 1992;30:433-40

Elwassif MM, Kong Q, Vazquez M, Bikson M. Bio-heat transfer model of deep brain stimulation-induced temperature changes. J Neural Eng 2006;3:306-15.

Enomoto H, Ugawa Y, Hanajima R, Yuasa K, Mochizuki H, Terao Y, et al. Decreased sensory cortical excitability after $1 \mathrm{~Hz}$ rTMS over the ipsilateral primary motor cortex. Clin Neurophysiol 2001;112:2154-8.

Epstein CM. Seizure or convulsive syncope during 1-Hz rTMS? Clin Neurophysiol 2006;117:2566-8.

Epstein CM, Davey KR. Iron-core coils for transcranial magnetic stimulation. J Clin Neurophysiol 2002;19:376-81.

Evers S, Böckermann I, Nyhuis PW. The impact of transcranial magnetic stimulation on cognitive processing: an event-related potential study. Neuroreport 2001a;12:2915.

Evers S, Hengst K, Pecuch PW. The impact of repetitive transcranial magnetic stimulation on pituitary hormone levels and cortisol in healthy subjects. J Affect Disord 2001b;66:83-8.

Figiel GS, Epstein C, McDonald WM, Amazon-Leece J, Figiel L, Saldivia A, et al. The use of rapid-rate transcranial magnetic stimulation (rTMS) in refractory depressed patients. J Neuropsychiat Clin Neurosci 1998;10:20-5.

Filippi MM, Oliveri M, Vernieri F, Pasqualetti P, Rossini PM. Are autonomic signals influencing cortico-spinal motor excitability? A study with transcrania magnetic stimulation. Brain Res 2000;881:159-64.

Fitzgerald PB, Brown TL, Marston NA, Oxley T, De Castella A, Daskalakis ZJ, et al. Reduced plastic brain responses in schizophrenia: a transcranial magnetic stimulation study. Shizophrenia Res 2004;71:17-26.

Fitzgerald PB, Benitez J, de Castella A, Daskalakis ZJ, Brown TL, Kulkarni J. A randomized, controlled trial of sequential bilateral repetitive transcrania magnetic stimulation for treatment-resistant depression. Am J Psychiat 2006;163:88-94

Fitzgerald PB, Hoy K, McQueen S, Maller JJ, Herring S, Segrave R, et al. A randomized trial of rTMS targeted with MRI based neuro-navigation in treatment-resistant depression. Neuropsychopharmacology 2009;34:1255-62.

Flitman SS, Grafman J, Wassermannn EM, Cooper V, O'Grady J, Pascual-Leone A et al. Linguistic processing during repetitive transcranial magnetic stimulation. Neurology 1998;50:175-81.

Foerster A, Schmitz JM, Nouri S, Claus D. Safety of rapid-rate transcranial magnetic stimulation: heart rate and blood pressure changes. Electroencephalogr Clin Neurophysiol 1997;104:207-12.

Folmer RL, Carroll JR, Rahim A, Shi Y, Hal Martin W. Effects of repetitive transcranial magnetic stimulation (rTMS) on chronic tinnitus. Acta Otolaryngol 2006;556(Suppl.):96-101.

Fregni F, Pascual-Leone AP. Technology insight: noninvasive brain stimulation in neurology-perspectives on the therapeutic potential of rTMS and tDCS. Nat Clin Pract Neurol 2007;3:383-93.

Fregni F, Simon DK, Wu A, Pascual-Leone A. Non-invasive brain stimulation for Parkinson's disease: a systematic review and meta-analysis of the literature. J Neurol Neurosurg Psychiat 2005a;76:1614-23.

Fregni F, Thome-Souza S, Bermpohl F, Marcolin MA, Herzog A, Pascual-Leone A et al. Antiepileptic effects of repetitive transcranial magnetic stimulation in patients with cortical malformations: an EEG and clinical study. Stereotact Funct Neurosurg 2005b;83:57-62.

Fregni F, Boggio PS, Valle AC, Rocha RR, Duarte J, Ferreira MJ, et al. A Shamcontrolled trial of a 5-day course of repetitive transcranial magnetic stimulation of the unaffected hemisphere in stroke patients. Stroke 2006a;37:2115-22.

Fregni F, Otachi PT, Do Valle A, Boggio PS, Thut G, Rigonatti SP, et al. A randomized clinical trial of repetitive transcranial magnetic stimulation in patients with refractory epilepsy. Ann Neurol 2006b;60:447-55.

Frye RE, Rotenberg A, Ousley M, Pascual-Leone A. Transcranial magnetic stimulation in child neurology: current and future directions. J Child Neurol 2008;23: 79-96.

Fuggetta G, Fiaschi A, Manganotti P. Modulation of cortical oscillatory activities induced by varying single-pulse transcranial magnetic stimulation intensity over the left primary motor area: a combined EEG and TMS study. Neuroimage 2005;27:896-908.

Fuggetta G, Pavone EF, Walsh V, Kiss M, Eimer M. Cortico-cortical interactions in spatial attention: a combined ERP/TMS study. J Neurophysiol 2006;95:3277-80.

Gandhi OP. Electromagnetic fields: human safety issues. Annu Rev Biomed Eng 2002;4:211-34.

Gates JR, Dhuna A, Pascual-Leone A. Lack of pathologic changes in human temporal lobes after transcranial magnetic stimulation. Epilepsia 1992;33:504-8.
Gentner R, Wankerl K, Reinsberger C, Zeller D, Classen J. Depression of human corticospinal excitability induced by magnetic theta-burst stimulation: evidence of rapid polarity-reversing metaplasticity. Cer Cortex 2008;19:2053-6.

George MS, Wassermannn EM, Williams WA, Steppel J, Pascual-Leone A, Basser P. et al. Changes in mood and hormone levels after rapid-rate transcranial magnetic stimulation (rTMS) of the prefrontal cortex. J Neuropsychiat Clin Neurosci 1996;8:172-80.

George MS, Nahas Z, Borckardt JJ, Anderson B, Foust MJ, Burns C, et al. Brain stimulation for the treatment of psychiatric disorders. Curr Opin Psychiat 2007;20:250-4.

Gerschlager W, Siebner HR, Rothwell JC. Decreased corticospinal excitability after subthreshold $1 \mathrm{~Hz}$ rTMS over lateral premotor cortex. Neurology 2001;57:449-55.

Gershon AA, Dannon PN, Grunhaus L. Transcranial magnetic stimulation in the treatment of depression. Am J Psychiat 2003;160:835-45.

Gilbert DL, Garvey MA, Bansal AS, Lipps T, Zhang J, Wassermannn EM. Should transcranial magnetic stimulation research in children be considered minimal risk? Clin Neurophysiol 2004;115:1730-9.

Green RM, Pascual-Leone A, Wassermann EM. Ethical guidelines for rTMS research. IRB 1997;2:1-7.

Grossheinrich N, Rau A, Pogarell O, Hennig-Fast K, Reinl M, Karch S, Dieler A, et al. Theta burst stimulation of the prefrontal cortex: safety and impact on cognition, mood, and resting electroencephalogram. Biol Psychiat 2009;65: 778-84.

Gugino LD, Romero JR, Aglio L, Titone D, Ramirez M, Pascual-Leone A, et al. Transcranial magnetic stimulation coregistered with MRI: a comparison of a guided vs. blind stimulation technique and its effect on evoked compound muscle action potentials. Clin Neurophysiol 2001;112:1781-92.

Hallett M. Transcranial Magnetic Stimulation: A Primer. Neuron 2007;55:187-99.

Hallett M, Wassermann EM, Pascual-Leone A, Valls-Solé J. Repetitive transcranial magnetic stimulation. Recommendations for the practice of clinical neurophysiology: guidelines of the international federation of clinical neurophysiology. In: Deuschl G, Eisen A, editors. Electroencephalography and clinical neurophysiology, 2nd ed. (Suppl. 52); 1999. p. 105-113.

Hamada M, Hanajima R, Terao Y, Arai N, Furubayashi T, Inomata-Terada S, et al. Origin of facilitation in repetitive, $1.5 \mathrm{~ms}$ interval, paired pulse transcranial magnetic stimulation (rPPS) of the human motor cortex. Clin Neurophysiol 2007;118:1596-601.

Hamada M, Terao Y, Hanajima R, Shirota Y, Nakatani-Enomoto S, Furubayashi T, Matsumoto H, Ugawa Y. Bidirectional long-term motor cortical plasticity and metaplasticity induced by quadripulse transcranial magnetic stimulation. J Physiol 2008;586:3927-47.

Hansenne M, Laloyaux O, Mardaga S, Ansseau M. Impact of low frequency transcranial magnetic stimulation on event-related brain potentials. Biol Psychol 2004;67:331-41.

Harris J, Clifford C, Miniussi C. The functional effect of transcranial magnetic stimulation: signal suppression or neural noise generation? J Cogn Sci 2008;20:734-40.

Haupts MR, Daum S, Ahle G, Holinka B, Gehlen W. Transcranial magnetic stimulation as a provocation for epileptic seizures in multiple sclerosis. Mult Scler 2004;10:475-6.

Hausmann A, Marksteiner J, Hinterhuber H, Humpel C. Magnetic stimulation induces neuronal c-fos via tetrodotoxin-sensitive sodium channels in organotypic cortex brain slices of the rat. Neurosci Lett 2001;310:105-8.

Herwig U, Fallgatter AJ, Höppner J, Eschweiler GW, Kron M, Hajak G, et al. Antidepressant effects of augmentative transcranial magnetic stimulation: randomised multicentre trial. Br J Psychiat 2007;191:441-8.

Hidding U, Bäumer T, Siebner HR, Demiralay C, Buhmann C, Weyh T, et al. MEP latency shift after implantation of deep brain stimulation systems in the subthalamic nucleus in patients with advanced Parkinson's disease. Mov Disord 2006;21:1471-6.

Hill DL, McLeish K, Keevil SF. Impact of electromagnetic field exposure limits in Europe: is the future of interventional MRI safe? Acad Radiol 2005;12: 1135-42.

Hoffman RE, Gueorguieva R, Hawkins KA, Varanko M, Boutros NN, Wu YT, et al. Temporoparietal transcranial magnetic stimulation for auditory hallucinations: safety, efficacy and moderators in a fifty patient sample. Biol Psychiat 2005;58:97-104.

Hoffman RE, Hampson M, Wu K, Anderson AW, Gore JC, Buchanan RJ, et al. Probing the pathophysiology of auditory/verbal hallucinations by combining functional magnetic resonance imaging and transcranial magnetic stimulation. Cereb Cortex 2007; 17:2733-43.

Holler I, Siebner HR, Cunnington R, Gerschlager W. 5 Hz repetitive TMS increases anticipatory motor activity in the human cortex. Neurosci Lett 2006;392:221-5.

Hsu KH, Nagarajan SS, Durand DM. Analysis of efficiency of magnetic stimulation. IEEE Trans Biomed Eng 2003;50:1276-85.

Huang YZ, Edwards MJ, Rounis E, Bhatia KP, Rothwell JC. Theta burst stimulation of the human motor cortex. Neuron 2005;45:201-6.

Huber R, Esser SK, Ferrarelli F, Massimini M, Peterson MJ, Tononi G. TMS-induced cortical potentiation during wakefulness locally increases slow wave activity during sleep. PLoS ONE 2007;2:e276.

Hufnagel A, Elger CE. Responses of the epileptic focus to transcranial magnetic stimulation. Electroencephalogr Clin Neurophysiol 1991;43(Suppl.):86-99.

International Commission on Non-ionizing Radiation Protection. Guidance on determining compliance of exposure to pulsed and complex non-sinusoidal 
waveforms below $100 \mathrm{kHz}$ with ICNIRP guidelines. Health Phys 2003;84:38387.

Iezzi E, Conte A, Suppa A, Agostino R, Dinapoli L, Scontrini A, et al. Phasic voluntary movements reverse the aftereffects of subsequent theta-burst stimulation in humans. J Neurophysiol 2008;100:2070-6.

Illes J, Gallo M, Kirschen MP. An ethics perspective on transcranial magnetic stimulation (TMS) and human neuromodulation. Behav Neurol 2006;7:149-57.

Ilmoniemi RJ, Virtanen J, Ruohonen J, Karhu J, Aronen HJ, Näätänen R, et al. Neuronal responses to magnetic stimulation reveal cortical reactivity and connectivity. Neuroreport 1997;8:3537-40.

Inghilleri M, Conte A, Currà A, Frasca V, Lorenzano C, Berardelli A. Ovarian hormones and cortical excitability. An rTMS study in humans. Clin Neurophysiol 2005; $115: 1063-8$

Ishikawa S, Matsunaga K, Nakanishi R, Kawahira K, Murayama N, Tsuji S, et al. Effect of theta burst stimulation over the human sensorimotor cortex on motor and somatosensory evoked potentials. Clin Neurophysiol 2007;118:1033-43.

Ives JR, Rotenberg A, Poma R, Thut G, Pascual-Leone A. Electroencephalographic recording during transcranial magnetic stimulation in humans and animals. Clin Neurophysiol 2006;117:1870-5.

Iyer MB, Schleper N, Wassermannn EM. Priming stimulation enhances the depressant effect of low-frequency repetitive transcranial magnetic stimulation. J Neurosci 2003;23:10867-72.

Jahanshahi M, Ridding MC, Limousin P, Profice P, Fogel W, Dressler D, et al. Rapid rate transcranial magnetic stimulation - a safety study. Electroencephalogr Clin Neurophysiol 1997;105:422-9.

Janicak PG, O'Reardon JP, Sampson SM, Husain MM, Lisanby SH, Rado JT, et al. Transcranial magnetic stimulation in the treatment of major depressive disorder: a comprehensive summary of safety experience from acute exposure, extended exposure, and during reintroduction treatment. J Clin Psychiat 2008;69:222-32.

Jennum P, Winkel H, Fuglsang-Frederiksen A, Dam M. EEG changes following repetitive transcranial magnetic stimulation in patients with temporal lobe epilepsy. Epilepsy Res 1994;18:167-73.

Jing H, Takigawa M, Okamura H, Doi W, Fukuzako H. Comparisons of event-related potentials after repetitive transcranial magnetic stimulation. J Neurol 2001;248:184-92.

Joo EY, Han SJ, Chung SH, Cho JW, Seo DW, Hong SB. Antiepileptic effects of lowfrequency repetitive transcranial magnetic stimulation by different stimulation durations and locations. Clin Neurophysiol 2007;118:702-8.

Jorge RE, Robinson RG, Tateno A, Narushima K, Acion L, Moser D, et al. Repetitive transcranial magnetic stimulation as treatment of poststroke depression: a preliminary study. Biol Psychiat 2004;55:398-405.

Julkunen P, Pääkkönen A, Hukkanen T, Könönen M, Tiihonen P, Vanhatalo S, et al. Efficient reduction of stimulus artefact in TMS-EEG by epithelial shortcircuiting by mini-punctures. Clin Neurophysiol 2008;119:475-81.

Kähkönen S, Kesäniemi M, Nikouline VV, Karhu J, Ollikainen M, Holi M, et al. Ethanol modulates cortical activity: direct evidence with combined TMS and EEG. Neuroimage 2001;14:322-8

Kähkönen S, Wilenius J, Komssi S, Ilmoniemi RJ. Distinct differences in cortical reactivity of motor and prefrontal cortices to magnetic stimulation. Clin Neurophysiol 2004;115:583-8.

Kähkönen S, Komssi S, Wilenius J, Ilmoniemi RJ. Prefrontal transcranial magnetic stimulation produces intensity-dependent EEG responses in humans. Neuroimage 2005;24:955-60.

Kammer T, Beck S, Thielscher A, Laubis-Herrmann U, Topka H. Motor thresholds in humans: a transcranial magnetic stimulation study comparing different pulse waveforms, current directions and stimulator types. Clin Neurophysiol 2001;112:250-8.

Kanno M, Chuma T, Mano Y. Monitoring an electroencephalogram for the safe application of therapeutic repetitive transcranial magnetic stimulation. J Neurol Neurosurg Psychiat 2001;71:559-60.

Karlström EF, Lundström R, Stensson O, Mild KH. Therapeutic staff exposure to magnetic field pulses during TMS/rTMS treatments. Bioelectromagnetics 2006;27:156-8.

Katayama T, Rothwell JC. Modulation of somatosensory evoked potentials using transcranial magnetic intermittent theta burst stimulation. Clin Neurophysiol 2007;118:2506-11.

Keck ME, Sillaber I, Ebner K, Welt T, Toschi N, Kaehler ST, et al. Acute transcranial magnetic stimulation of frontal brain regions selectively modulates the release of vasopressin, biogenic amines and amino acids in the rat brain. Eur J Neurosci 2000; $12: 3713-20$

Kimbrell TA, Little JT, Dunn RT, Frye MA, Greenberg BD, Wassermann EM, et al. Frequency dependence of antidepressant response to left prefrontal repetitive transcranial magnetic stimulation (rTMS) as a function of baseline cerebral glucose metabolism. Biol Psychiat 1999;46:1603-13.

Klirova M, Novak T, Kopecek M, Mohr P, Strunzova V. Repetitive transcranial magnetic stimulation (rTMS) in major depressive episode during pregnancy. Neuro Endocrinol Lett 2008;29:69-70.

Kobayashi M, Pascual-Leone A. Transcranial magnetic stimulation in neurology. Lancet Neurol 2003;2:145-56.

Kofler M, Leis AA, Sherwood AM, Delapasse JS, Halter JA. Safety of transcranial magnetic stimulation in patients with abdominally implanted electronic devices. Lancet 1991;338:1275-6.

Komssi S, Aronen HJ, Huttunen J, Kesäniemi M, Soinne L, Nikouline VV, et al. Ipsiand contralateral EEG reactions to transcranial magnetic stimulation. Clin Neurophysiol 2002;113:175-84.
Komssi S, Kähkönen S, Ilmoniemi RJ. The effect of stimulus intensity on brain responses evoked by transcranial magnetic stimulation. Hum Brain Mapp 2004;21:154-64.

Kozel FA, Nahas Z, deBrux C, Molloy M, Lorberbaum JP, Bohning D, et al. How coilcortex distance relates to age, motor threshold, and antidepressant response to repetitive transcranial magnetic stimulation. J Neuropsychiat Clin Neurosci 2000;12:376-84.

Kruger B. An update on the external ear resonance in infants and young children. Ear Hear 1987;8:333-6.

Kübler A, Schmidt K, Cohen LG, Lotze M, Winter S, Hinterberger T, et al. Modulation of slow cortical potentials by transcranial magnetic stimulation in humans. Neurosci Lett 2002;324:205-8.

Kühn AA, Trottenberg T, Kupsch A, Meyer BU. Pseudo-bilateral hand motor responses evoked by transcranial magnetic stimulation in patients with deep brain stimulators. Clin Neurophysiol 2002;113:341-5.

Kühn AA, Brandt SA, Kupsch A, Trottenberg T, Brocke J, Irlbacher $\mathrm{K}$, et al Comparison of motor effects following subcortical electrical stimulation through electrodes in the globus pallidus internus and cortical transcranial magnetic stimulation. Exp Brain Res 2004;155:48-55.

Kujirai T, Sato M, Rothwell JC, Cohen LG. The effect of transcranial magnetic stimulation on median nerve somatosensory evoked potentials. Electroencephalogr Clin Neurophysiol 1993;89:227-34.

Kumar R, Chen R, Ashby P. Safety of transcranial magnetic stimulation in patients with implanted deep brain stimulators. Mov Disord 1999;14:157-8.

Lang N, Siebner H, Ernst D, Nitsche MA, Paulus W, Lemon RJ, et al. Preconditioning with transcranial direct current stimulation sensitizes the motor cortex to rapid-rate transcranial magnetic stimulation and controls the direction of aftereffects. Biol Psychiat 2004;56:634-9.

Leafaucheur JP. Transcranial magnetic stimulation in the management of pain Suppl Clin Neurophysiol 2004;57:737-48.

Lefaucheur JP, Drouot X, Von Raison F, Ménard-Lefaucheur I, Cesaro P, Nguyen JP. Improvement of motor performance and modulation of cortical excitability by repetitive transcranial magnetic stimulation of the motor cortex in Parkinson's disease. Clin Neurophysiol 2004;115:2530-41.

Lempert T. Recognizing syncope: pitfalls and surprises. J R Soc Med 1996;89: 372-5.

Levkovitz Y, Roth Y, Harel EV, Braw Y, Sheer A, Zangen A. A randomized controlled feasibility and safety study of deep transcranial magnetic stimulation. Clin Neurophysiol 2007;118:2730-44.

Li X, Nahas Z, Lomarev M, Denslow S, Shastri A, Bohning DE, et al. Prefrontal cortex transcranial magnetic stimulation does not change local diffusion: a magnetic resonance imaging study in patients with depression. Cogn Behav Neurol 2003; $16: 128-35$

Liebetanz D, Fauser S, Michaelis T, Czéh B, Watanabe T, Paulus W, et al. Safety aspects of chronic low-frequency transcranial magnetic stimulation based on localized proton magnetic resonance spectroscopy and histology of the rat brain. J Psychiat Res 2003;37:277-86.

Lin JT, Ziegler DK, Lai CW, Bayer W. Convulsive syncope in blood donors. Ann Neurol 1982;11:525-8

Lisanby $\mathrm{SH}$. Update on magnetic seizure therapy: a novel form of convulsive therapy. J ECT 2002;18:182-8.

Lisanby SH, Gutman D, Luber B, Schroeder C, Sackeim HA, Sham TMS. Intracerebral measurement of the induced electrical field and the induction of motor-evoked potentials. Biol Psychiat 2001;49:460-3.

Litvak V, Komssi S, Scherg M, Hoechstetter K, Classen J, Zaaroor M, et al. Artifact correction and source analysis of early electroencephalographic responses evoked by transcranial magnetic stimulation over primary motor cortex. Neuroimage 2007;37:56-70.

Lomarev MP, Kanchana S, Bara-Jimenez W, Iyer M, Wassermannn EM, Hallett M Placebo-controlled study of rTMS for the treatment of Parkinson's disease. Mov Dis 2006;21:325-31.

Lomarev MP, Kim DY, Richardson SP, Voller B, Hallett M. Safety study of highfrequency transcranial magnetic stimulation in patients with chronic stroke Clin Neurophysiol 2007;118:2072-5.

Lontis ER, Voigt M, Struijk JJ. Focality assessment in transcranial magnetic stimulation with double and cone coils. J Clin Neurophysiol 2006;23: 462-71.

Loo C, Sachdev P, Elsayed H, McDarmont B, Mitchell P, Wilkinson M, et al. Effects of a 2- to 4-week course of repetitive transcranial magnetic stimulation (rTMS) on neuropsychologic functioning, electroencephalogram, and auditory threshold in depressed patients. Biol Psychiat 2001;49:615-23.

Loo CK, Mitchell PB, Croker VM, Malhi GS, Wen W, Gandevia SC, et al. Double-blind controlled investigation of bilateral prefrontal transcranial magnetic stimulation for the treatment of resistant major depression [see comment] Psychol Med 2003;33:33-40.

Loo CK, McFarquhar TF, Mitchell PB. A review of the safety of repetitive transcranial magnetic stimulation as a clinical treatment for depression. Int J Neuropsychopharmacol 2008;11:131-47.

Lorenzano C, Gilio F, Inghilleri M, Conte A, Fofi L, Manfredi M, et al. Spread of electrical activity at cortical level after repetitive magnetic stimulation in normal subjects. Exp Brain Res 2002;147:186-92.

Lu M-K, Bliem B, Jung P, Arai N, Tsai C-H, Ziemann U. Modulation of preparatory volitional motor cortical activity by paired associative transcranial magnetic stimulation. Hum Brain Map 2009 [Epub ahead of print].

Luft AR, Kaelin-Lang A, Hauser TK, Cohen LG, Thakor NV, Hanley DF. Transcrania magnetic stimulation in the rat. Exp Brain Res 2001;140:112-21. 
Maccabee PJ, Amassian VE, Eberle LP, Cracco RQ. Magnetic coil stimulation of straight and bent amphibian and mammalian peripheral nerve in vitro: locus of excitation. J Physiol 1993;460:201-19.

Machii K, Cohen D, Ramos-Estebanez C, Pascual-Leone A. Safety of rTMS to nonmotor cortical areas in healthy participants and patients. Clin Neurophysiol 2006;117:455-71.

Magistris MR, Rösler KM, Truffert A, Myers JP. Transcranial stimulation excites virtually all motor neurons supplying the target muscle. A demonstration and a method improving the study of motor evoked potentials. Brain 1998;121:437-50

Magistris MR, Rösler KM, Truffert A, Landis T, Hess CW. A clinical study of motor evoked potentials using a triple stimulation technique. Brain 1999:122:265-79.

Málly J, Stone TW. New advances in the rehabilitation of CNS diseases applying rTMS. Expert Rev Neurother 2007;7:165-77.

Marg E, Rudiak D. Phosphenes induced by magnetic stimulation over the occipital brain: description and probable site of stimulation. Optom Vis Sci 1994;71:301-11.

Mariorenzi R, Zarola F, Caramia MD, Paradiso C, Rossini PM. Non-invasive evaluation of central motor tract excitability changes following periphera nerve stimulaiton in healthy humans. Electroenceph Clin Neurophysio $1991 ; 81: 243-50$.

Martens L. Different basic dosimetric quantities for the characterization of exposure to low-frequency electric and magnetic fields and the implication for practical exposure conditions and guidelines. Health Phys 2007;92:515-20.

Mashour GA, Walker EE, Martuza RL. Psychosurgery: past, present, and future. Brain Res Brain Res Rev 2005;48:409-19.

Massimini M, Ferrarelli F, Huber R, Esser SK, Singh H, Tononi G. Breakdown of cortical effective connectivity during sleep. Science 2005;309:2228-32.

Massimini M, Ferrarelli F, Esser SK, Riedner BA, Huber R, Murphy M, et al. Triggering sleep slow waves by transcranial magnetic stimulation. Proc Natl Acad Sci 2007;104:8496-501.

Matsumi N, Matsumoto K, Mishima N, Moriyama E, Furuta T, Nishimoto A, et al. Thermal damage threshold of brain tissue: histological study of heated normal monkey brains. Neurologia Medico-Chirurgica 1994;34:209-15.

Matsumiya Y, Yamamoto T, Yarita M, Miyauchi S, Kling JW. Physical and physiological specification of magnetic pulse stimuli that produce cortical damage in rats. J Clin Neurophysiol 1992;9:278-87.

May A, Hajak G, Gänssbauer S, Steffens T, Langguth B, Kleinjung T, et al. Structural brain alterations following 5 days of intervention: dynamic aspects of neuroplasticity. Cereb Cortex 2007;17:205-10.

Menkes DL, Gruenthal M. Slow-frequency repetitive transcranial magnetic stimulation in a patient with focal cortical dysplasia. Epilepsia 2000;41: 240-2.

Michael N, Gösling M, Reutemann M, Kersting A, Heindel W, Arolt V, et al. Metabolic changes after repetitive transcranial magnetic stimulation (rTMS) of the left prefrontal cortex: a Sham-controlled proton magnetic resonance spectroscopy (1H MRS) study of healthy brain. Eur J Neurosci 2003;17:2462-8.

Miranda PC, Hallett M, Basser PJ. The electric field induced in the brain by magnetic stimulation: a 3D finite-element analysis of the effect of tissue heterogeneity and anisotropy. IEEE Trans Biomed Eng 2003;50:1074-85.

Misawa S, Kuwabara S, Shibuya K, Mamada K, Hattori T. Low-frequency transcranial magnetic stimulation for epilepsia partialis continua due to cortical dysplasia. J Neurol Sci 2005;234:37-9.

Mistry S, Verin E, Singh S, Jefferson S, Rothwell JC, Thompson DG, et al. Unilateral suppression of pharyngeal motor cortex to repetitive transcranial magnetic stimulation reveals functional asymmetry in the hemispheric projections to human swallowing. J Physiol 2007;585:525-38.

Morbidi F, Garulli A, Prattichizzo D, Rizzo C, Manganotti P, Rossi S. Off-line removal of TMS-induced artifacts on human electroencephalography by Kalman filter. J Neurosci Methods 2007;162:293-302.

Mottaghy FM, Gangitano M, Horkan C, Chen Y, Pascual-Leone A, Schlaug G. Repetitive TMS temporarily alters brain diffusion. Neurology 2003;60:1539-41.

Muellbacher W, Ziemann U, Boroojerdi B, Hallett M. Effects of low-frequency transcranial magnetic stimulation on motor excitability and basic motor behavior. Clin Neurophysiol 2000;111:1002-7.

Münchau A, Bloem BR, Irlbacher K, Trimble MR, Rothwell JC. Functional connectivity of human premotor and motor cortex explored with repetitive transcranial magnetic stimulation. J Neurosci 2002;22:554-61.

Nadeem M, Thorlin T, Gandhi O, Persson M. Computation of electric and magnetic stimulation in human head using the 3D impedance method. IEEE Trans Biomed Eng 2003;50:900-7.

Nagarajan SS, Durand DM, Warman EN. Effects of induced electric fields on finite neuronal structures: a simulation study. IEEE Trans Biomed Eng 1993;40:1175-88.

Nahas Z, Bohning D, Molloy M, Oustz J, Risch C, George M. Safety and feasibility of repetitive transcranial magnetic stimulation in the treatment of anxiousdepression in pregnancy: a case report. J Clin Psychiat 1999;60:50-2.

Nahas Z, DeBrux C, Chandler V, Lorberbaum JP, Speer AM, Molloy MA, et al. Lack of significant changes on magnetic resonance scans before and after 2 weeks of daily left prefrontal repetitive transcranial magnetic stimulation for depression. J ECT 2000;16:380-90.

Neveu PJ, Barnéoud P, Vitiello S, Kelley KW, Le Moal MA. Brain neocortex modulation of mitogen-induced interleukin 2, but not interleukin production. Immunol Lett 1989;21:307-10.

Nikouline V, Ruohonen J, Ilmoniemi RJ. The role of the coil click in TMS assessed with simultaneous EEG. Clin Neurophysiol 1999;110:1325-8.
Nowak DA, Hoffmann U, Connemann BJ, Schonfeldt-Lecuona C. Epileptic seizure following $1 \mathrm{~Hz}$ repetitive transcranial magnetic stimulation. Clin Neurophysiol 2006; 117:1631-3.

Nyffeler T, Wurtz P, Lüscher HR, Hess CW, Senn W, Pflugshaupt T, et al. Extending lifetime of plastic changes in the human brain. Eur J Neurosci 2006;24:2961-6.

Obermann LM, Pascual-Leone A. Report of seizure induced by continuous theta burst stimulation. Brain Stim 2009, in press.

O'Reardon J, Solvason B, Janicak P, Sampson S, Isenberg K, Nahas Z, et al. Efficacy and safety of transcranial magnetic stimulation in the acute treatment of major depression: a multi-site randomized controlled trial. Biol Psychiat 2007;62:1208-16.

O'Shea J, Johansen-Berg H, Trief D, Göbel S, Rushworth MF. Functionally specific reorganization in human premotor cortex. Neuron 2007;54:479-90.

Padberg F, di Michele F, Zwanzger P, Romeo E, Bernardi G, Schüle C, et al. Plasma concentrations of neuroactive steroids before and after repetitive transcranial magnetic stimulation (rTMS) in major depression. Neuropsychopharmacology 2002;27:874-8.

Pascual-Leone A, Gates JR, Dhuna A. Induction of speech arrest and counting errors with rapid-rate transcranial magnetic stimulation. Neurology 1991;41:697-702.

Pascual-Leone A, Cohen LG, Shotland LI, Dang N, Pikus A, Wassermannn EM, et al. No evidence of hearing loss in humans due to transcranial magnetic stimulation. Neurlology 1992;42:647-51.

Pascual-Leone A, Houser CM, Reese K, Shotland LI, Grafman J, Sato S. Valls-Sol_ J, Brasil-Neto JP, Wassermannn EM, Cohen LG. Safety of rapid-rate transcranial magnetic stimulation in normal volunteers. Electroencephalogr Clin Neurophysiol 1993;89:120-30.

Paus T, Sipila PK, Strafella AP. Synchronization of neuronal activity in the human primary motor cortex by transcranial magnetic stimulation: an EEG study. J Neurophysiol 2001;86:1983-90.

Peinemann A, Reimer B, Löer C, Quartarone A, Münchau A, Conrad B, Siebner HR. Long-lasting increase in corticospinal excitability after 1800 pulses of subthreshold $5 \mathrm{~Hz}$ repetitive TMS to the primary motor cortex. Clin Neurophysiol 2004;115:1519-26.

Penfield W, Jasper $H$. Epilepsy and the functional anatomy of the human brain. Boston, MA: Little, Brown; 1954

Peterchev AV, Jalinous R, Lisanby SH. A transcranial magnetic stimulator inducing near-rectangular pulses with controllable pulse width (cTMS). IEEE Trans Biomed Eng 2008;55:257-66.

Post A, Keck ME. Transcranial magnetic stimulation as a therapeutic tool in psychiatry: what do we know about the neurobiological mechanisms? J. Psychiatr. Res. 2001;35:193-215.

Price GW. EEG-dependent ERP recording: using TMS to increase the incidence of a selected pre-stimulus pattern. Brain Res. Brain Res. Protoc. 2004;12:144-51.

Prikryl R, Kucerova $H$. Occurrence of epileptic paroxysm during repetitive transcranial magnetic stimulation treatment. J. Psychopharmacol. 2005;19:313.

Quartarone A, Rizzo V, Bagnato S, Morgante F, Sant'Angelo A, Girlanda P, Siebner HR. Rapid-rate paired associative stimulation of the median nerve and motor cortex can produce long-lasting changes in motor cortical excitability in humans. J Physiol. 2006;575:657-70.

Ray PG, Meador KJ, Epstein CM, Loring DW, Day LJ. Magnetic stimulation of visual cortex: factors influencing the perception of phosphenes. J Clin Neurophysiol 1998; $15: 351-7$.

Restuccia D, Ulivelli M, De Capua A, Bartalini S, Rossi S. Modulation of highfrequency $(600 \mathrm{~Hz})$ somatosensory-evoked potentials after rTMS of the primary sensory cortex. Eur J Neurosci 2007;26:2349-58.

Riches SF, Collins DJ, Scuffham JW, Leach MO. EU Directive 2004/40: field measurements of a 1.5 T clinical MR scanner. Br J Radiol 2007a;80:483-7.

Riches SF, Collins DJ, Charles-Edwards GD, Shafford JC, Cole J, Keevil SF, et al. Measurements of occupational exposure to switched gradient and spatiallyvarying magnetic fields in areas adjacent to $1.5 \mathrm{~T}$ clinical MRI systems. J Magn Reson Imag 2007b;26:1346-52.

Ridding MC, Rothwell JC. Is there a future for therapeutic use of transcranial magnetic stimulation? Nat Rev Neurosci 2007;8:559-67.

Robertson EM, Théoret $\mathrm{H}$, Pascual-Leone A. Studies in cognition: the problems solved and created by transcranial magnetic stimulation. J Cogn Neurosci 2003;15:948-60.

Romei V, Brodbeck V, Michel C, Amedi A, Pascual-Leone A, Thut G. Spontaneous fluctuations in posterior alpha-band EEG activity reflect variability in excitability of human visual areas. Cereb Cortex 2008a;18:2010-8.

Romei V, Rihs T, Brodbeck V, Thut G. Resting electroencephalogram alpha-power over posterior sites indexes baseline visual cortex excitability. Neuroreport 2008b;19:203-8.

Rosa MA, Odebrecht M, Rigonatti SP, Marcolin MA. Transcranial magnetic stimulation: review of accidental seizures. Rev Bras Psiquiatr 2004:26:131-4.

Rossi S, Rossini PM. TMS in cognitive plasticity and the potential for rehabilitation. Trends Cogn Sci 2004;8:273-9.

Rossi S, Pasqualetti P, Rossini PM, Feige B, Ulivelli M, Glocker FX, et al. Effects of repetitive transcranial magnetic stimulation on movement-related cortical activity in humans. Cereb Cortex 2000;10:802-8

Rossi S, Cappa SF, Babiloni C, Pasqualetti P, Miniussi C, Carducci F, et al. Prefrontal cortex in long-term memory: an "interference" approach using magnetic stimulation. Nat Neurosci 2001;4:948-52.

Rossi S, Ulivelli M, Bartalini S, Galli R, Passero S, Battistini N, et al. Reduction of cortical myoclonus-related epileptic activity following slow-frequency rTMS. Neuroreport 2004a;15:293-6. 
Rossi S, Miniussi C, Pasqualetti P, Babiloni C, Rossini PM, Cappa SF. Age-related functional changes of prefrontal cortex in long-term memory: a repetitive transcranial magnetic stimulation study. J Neurosci 2004b;24:7939-44.

Rossi S, Pasqualetti P, Zito G, Vecchio F, Cappa SF, Miniussi C, et al. Prefrontal and parietal cortex in human episodic memory: an interference study by repetitive transcranial magnetic stimulation. Eur J Neurosci 2006;23:793-800.

Rossi S, De Capua A, Ulivelli M, Bartalini S, Falzarano V, Filippone G, et al. Effects of repetitive transcranial magnetic stimulation on chronic tinnitus. A randomized, cross-over, double-blind, placebo-controlled study. J Neurol Neurosurg Psychiat 2007a;78:857-63.

Rossi S, Ferro M, Cincotta M, Ulivelli M, Bartalini S, Miniussi C, et al. A real electromagnetic placebo (REMP) device for sham transcranial magnetic stimulation (TMS). Clin Neurophysiol 2007b;118:709-16.

Rossi S, Cappa SF, Rossini PM. Higher cognitive functions: memory and reasoning. In: Epstein C, Ziemann U, Wassermannn EM, Walsh V, Paus T, Lisanby SH, editors. Oxford handbook of transcranial stimulation. London: Oxford University Press; 2007c. p. 501-16.

Rossini PM, Rossi S. Transcranial magnetic stimulation: diagnostic, therapeutic and research potential. Neurology 2007;68:484-8.

Rossini PM, Desiato MT, Caramia MD. Age-related changes of motor evoked potentials in healthy humans: non-invasive evaluation of central and peripheral motor tracts conductivity and excitability. Brain Res 1992;593:14-9.

Rossini PM, Barker AT, Berardelli A, Caramia MD, Caruso G, Cracco RQ, et al. Noninvasive electrical and magnetic stimulation of the brain, spinal cord and roots: basic principles and procedures for routine clinical application. Report of an IFCN committee. Electroencephalogr Clin Neurophysiol 1994:91:79-92.

Rossini PM, Rossi S, Babiloni C, Polich J. Clinica neurophysiology of ging brain: from normal aging to neurodegeneration. Prog Neurobiol 2007;83:375-400.

Rotenberg A, Harrington MG, Birnbaum DS, Madsen JR, Glass LES, Jensen FE, et al. Minimal heating of titanium skull plates during $1 \mathrm{~Hz}$ repetitive transcranial magnetic stimulation. Clin Neurophysiol 2007;118:2536-8.

Rotenberg A, Hjunji Bae E, Takeoka M, Tormos JM, Schachter SC, Pascual-Leone AP. Repetitive transcranial magnetic stimulation in the treatment of epilepsia partialis continua. Epilepsy Behav 2009;14:253-7.

Roth BJ, Basser PJ. A model of the stimulation of a nerve fiber by electromagnetic induction. IEEE Trans Biomed Eng 1990;37:588-97.

Roth BJ, Pascual Leone A, Cohen LG, Hallett M. The heating of metal-electrodes during rapid-rate magnetic stimulation - a possible safety hazard. Electroenceph Clin Neurophysiol 1992;85:116-23.

Roth Y, Zangen A, Hallett M. A coil design for transcranial magnetic stimulation of deep brain regions. J Clin Neurophysiol 2002;19:361-70.

Roth Y, Amir A, Levkovitz Y, Zangen A. Three-dimensional distribution of the electric field induced in the brain by transcranial magnetic stimulation using figure 8 and deep h-coils. J Clin Neurophysiol 2007;24:31-8.

Ruohonen J, Ollikainen M, Nikouline V, Virtanen J, Ilmoniemi RJ. Coil design for real and sham transcranial magnetic stimulation. IEEE Trans Biomed Eng 2000;47:145-8.

Ruohonen J, Ilmoniemi RJ. Physical principles for transcranial magnetic stimulation. In: Pascual-Leone A, Davey NJ, Rothwell J, Wassermann EM, Puri BK, editors. Handbook of transcranial magnetic stimulation. New York: Oxford University Press; 2002.

Sack AT, Cohen Kadosh R, Schuhmann T, Moerel M, Walsh V, Goebel R. Optimizing functional accuracy of TMS in cognitive studies: a comparison of methods. J Cogn Neurosci 2009;21:207-21.

Santiago-Rodríguez E, Cárdenas-Morales L, Harmony T, Fernández-Bouzas A, Porras-Kattz E, Hernández A. Repetitive transcranial magnetic stimulation decreases the number of seizures in patients with focal neocortical epilepsy. Seizure 2008;17:677-83.

Satow T, Mima T, Hara H, Oga T, Ikeda A, Hashimoto N, et al. Nausea as a complication of low-frequency repetitive transcranial magnetic stimulation of the posterior fossa. Clin Neurophysiol 2002;113:1441-3.

Satow T, Mima T, Yamamoto J, Oga T, Begum T, Aso T, et al. Short-lasting impairment of tactile perception by $0.9 \mathrm{~Hz}-\mathrm{rTMS}$ of the sensorimotor cortex. Neurology 2003;60:1045-7.

Schrader LM, Stern JM, Koski L, Nuwer MR, Engel Jr J. Seizure incidence during single- and paired-pulse transcranial magnetic stimulation (TMS) in individuals with epilepsy. Clin Neurophysiol 2004;115:2728-37.

Schrader LM, Stern JM, Fields TA, Nuwer MR, Wilson CL. A lack of effect from transcranial magnetic stimulation (TMS) on the vagus nerve stimulator (VNS). Clin Neurophysiol 2005;116:2501-4

Schulze-Bonhage A, Scheufler K, Zentner J, Elger CE. Safety of single and repetitive focal transcranial magnetic stimuli as assessed by intracranial EEG recordings in patients with partial epilepsy. J Neurol 1999;246:914-9.

Schürmann M, Nikouline VV, Soljanlahti S, Ollikainen M, Basar E, Ilmoniemi RJ. EEG responses to combined somatosensory and transcranial magnetic stimulation. Clin Neurophysiol 2001;112:19-24.

Schutter DJ, van Honk J, d'Alfonso AA, Peper JS, Panksepp J. High frequency repetitive transcranial magnetic over the medial cerebellum induces a shift in the prefrontal electroencephalography gamma spectrum: a pilot study in humans. Neurosci Lett 2003;336:73-6.

Sgro J, Stanton P, Emerson R. Theoretical and practical performance of magnetic stimulators and coils. Electroencephalogr Clin Neurophysiol 1991;43(Suppl.):279-83.

Sibon I, Strafella AP, Gravel P, Ko JH, Booij L, Soucy JP, et al. Acute prefrontal cortex TMS in healthy volunteers: effects on brain 11C-alphaMtrp trapping. Neuroimage 2007;34:1658-64.
Siebner HR, Lang N, Rizzo V, Nitsche MA, Paulus W, Lemon RN, et al. Preconditioning of low-frequency repetitive transcranial magnetic stimulation with transcranial direct current stimulation: evidence for homeostatic plasticity in the human motor cortex. J Neurosci 2004;24:3379-85.

Siebner HR, Peller M, Lee L. TMS and positron emission tomography: methods and current advances. In: Wassermann EM, Epstein CM, Ziemann U, Walsh V, Paus $\mathrm{T}$, Lisanby $\mathrm{SH}$, editors. The Oxford handbook of transcranial magnetic stimulation. Oxford: Oxford University Press; 2008. p. 549-67.

Siebner HR, Bergmann TO, Bestmann S, Massimini M, Johansen-Berg H, Mochizuki $\mathrm{H}$, et al. Consensus paper: combining transcranial stimulation with neuroimaging. Brain Stimul 2009;2:58-80.

Silvanto J, Pascual-Leone A. State-dependency of transcranial magnetic stimulation. Brain Topogr 2008;21:1-10.

Silvanto J, Muggleton NG, Cowey A, Walsh V. Neural activation state determines behavioral susceptibility to modified theta burst transcranial magnetic stimulation. Eur J Neurosci 2007;26:523-8.

Silverstein FS, Jensen FE. Neonatal seizures. Ann Neurol 2007;62:112-20.

Smith MJ, Keel JC, Greenberg BD, Adams LF, Schmidt PJ, Rubinow DA, et al Menstrual cycle effects on cortical excitability. Neurology 1999;53: 2069-72.

Sommer M, Tergau F, Wischer S, Paulus W. Paired-pulse repetitive transcranial magnetic stimulation of the human motor cortex. Exp Brain Res 2001;139:465-72.

Sommer J, Jansen A, Drager BOS, Breitenstein C, Deppe M, Knecht S. Transcranial magnetic stimulation - a sandwich coil desing for a better Sham. Clin Neurophysiol 2006a;117:440-6.

Sommer M, Alfaro A, Rummel M, Speck S, Lang N, Tings T, et al. Half sine, monophasic and biphasic transcranial magnetic stimulation of the human motor cortex [see comment]. Clin Neurophysiol 2006b;117:838-44.

Steinhoff BJ, Stodieck SR, Zivcec Z, Schreiner R, von Maffei C, Plendl H, et al Transcranial magnetic stimulation (TMS) of the brain in patients with mesiotemporal epileptic foci. Clin Electroencephalogr 1993;24:1-5.

Steven MS, Pascual-Leone A. Transcranial magnetic stimulation and the human brain: an ethical evaluation. In: Illes J, editor. 21st Century neuroethics: defining the issues in research, practice and policy. Oxford, UK: Oxford University Press; 2006. p. 201-11.

Stewart LM, Walsh V, Rothwell JC. Motor and phosphene thresholds: a transcranial magnetic stimulation correlation study. Neuropsychologia 2001;39:415-9.

Strafella AP, Paus T, Barrett J, Dagher A. Repetitive transcranial magnetic stimulation of the human prefrontal cortex induces dopamine release in the caudate nucleus. J Neurosci 2001;21:RC157.

Strens LH, Oliviero A, Bloem BR, Gerschlager W, Rothwell JC, Brown P. The effects of subthreshold $1 \mathrm{~Hz}$ repetitive TMS on cortico-cortical and interhemispheric coherence. Clin Neurophysiol 2002;113:1279-85.

Szuba MP, O'Reardon JP, Rai AS, Snyder-Kastenberg J, Amsterdam JD, Gettes DR, et al. Acute mood and thyroid stimulating hormone effects of transcranial magnetic stimulation in major depression. Biol Psychiat 2001;50:22-7.

Tassinari CA, Cincotta M, Zaccara G, Michelucci R. Transcranial magnetic stimulation and epilepsy. Clin Neurophysiol 2003;114:777-98.

Tergau F, Neumann D, Rosenow F, Nitsche MA, Paulus W, Steinhoff B. Can epilepsies be improved by repetitive transcranial magnetic stimulation? Interim analysis of a controlled study. Clin Neurophysiol 2003(Suppl. 56):400-5.

Tharayil BS, Gangadhar BN, Thirthalli J, Anand L. Seizure with single-pulse transcranial magnetic stimulation in a 35-year-old otherwise-healthy patient with bipolar disorder. J ECT 2005;21:188-9.

Theodore WH, Hunter K, Chen R, Vega-Bermudez F, Boroojerdi B, Reeves-Tyer P, et al. Transcranial magnetic stimulation for the treatment of seizures: a controlled study. Neurology 2002;59:560-2.

Theoret H, Kobayashi M, Valero-Cabre A, Pascual-Leone A. Exploring paradoxical functional facilitation with TMS. Clin Neurophysiol 2003;56(Suppl.):211-9.

Thickbroom GW, Byrnes ML, Edwards DJ, Mastaglia FL. Repetitive paired-pulse TMS at I-wave periodicity markedly increases corticospinal excitability: a new technique for modulating synaptic plasticity. Clin Neurophysio 2006;117:61-6.

Thielscher A, Kammer T. Linking physics with physiology in TMS: a sphere field model to determine the cortical stimulation site in TMS. Neuroimage 2002;17:1117-30.

Thielscher A, Kammer T. Electric field properties of two commercial figure-8 coils in TMS: calculation of focality and efficiency. Clin Neurophysiol 2004;115:1697-708.

Thut G, Northoff G, Ives JR, Kamitani Y, Pfennig A, Kampmann F, et al. Effects of single-pulse transcranial magnetic stimulation (TMS) on functional brain activity: a combined event-related TMS and evoked potential study. Clin Neurophysiol 2003a;114:2071-80.

Thut G, Théoret H, Pfennig A, Ives J, Kampmann F, Northoff G, et al. Differential effects of low-frequency rTMS at the occipital pole on visual-induced alpha desynchronization and visual-evoked potentials. Neuroimage 2003b;18:334-47.

Thut G, Ives JR, Kampmann F, Pastor MA, Pascual-Leone A. A new device and protocol for combining TMS and online recordings of EEG and evoked potentials. J Neurosci Methods 2005;141:207-17.

Tiitinen H, Virtanen J, Ilmoniemi RJ, Kamppuri J, Ollikainen M, Ruohonen J, et al. Separation of contamination caused by coil clicks from responses elicited by transcranial magnetic stimulation. Clin Neurophysiol 1999;110:982-5.

Tofts PS. The distribution of induced currents in magnetic stimulation of the brain Phys Med Biol 1990;35:1119-28. 
Tsuji T, Rothwell JC. Long lasting effects of rTMS and associated peripheral sensory input on MEPs, SEPs and transcortical reflex excitability in humans. J Physiol 2002;540:367-76

Udupa K, Sathyaprabha TN, Thirthalli J, Kishore KR, Raju TR, Gangadhar BN Modulation of cardiac autonomic functions in patients with major depression treated with repetitive transcranial magnetic stimulation. J Affect Disord 2007; 104:231-6.

Valero-Cabré A, Pascual-Leone A, Rushmore RJ. Cumulative sessions of repetitive transcranial magnetic stimulation (rTMS) build up facilitation to subsequent TMS-mediated behavioural disruptions. Eur J Neurosci 2008;27:765-74.

Van Der Werf YD, Paus T. The neural response to transcranial magnetic stimulation of the human motor cortex. I. Intracortical and cortico-cortical contributions. Exp Brain Res 2006;175:231-45.

Van Der Werf YD, Sadikot AF, Strafella AP, Paus T. The neural response to transcranial magnetic stimulation of the human motor cortex. II. Thalamocortical contributions. Exp Brain Res 2006;175:246-55.

Vernieri F, Maggio P, Tibuzzi F, Filippi MM, Pasqualetti P, Melgari JM et al. High frequency repetitive transcranial magnetic stimulation decreases cerebralvasomotor reactivity. Clin Neurophysiol 2009 [Epub ahead of print].

Wagner TA, Zahn M, Grodzinsky AJ, Pascual-Leone A. Three-dimensional head model simulation of transcranial magnetic stimulation. IEEE Trans Biomed Eng 2004;51:1586-98.

Wagner T, Fregni F, Eden U, Ramos-Estebanez C, Grodzinsky A, Zahn M, et al. Transcranial magnetic stimulation and stroke: a computer-based human model study. Neuroimage 2006;30:857-70.

Wagner T, Valero-Cabre A, Pascual-Leone A. Noninvasive human brain stimulation. Annu Rev Biomed Eng 2007;9:527-65.

Wagner T, Eden U, Fregni F, Valero-Cabre A, Ramos-Estebanez C, Pronio-Stelluto V et al. Transcranial magnetic stimulation and brain atrophy: a computer-based human brain model study. Exp Brain Res 2008;186:539-50.

Wassermannn EM. Risk and safety of repetitive transcranial magnetic stimulation: report and suggested guidelines from the International Workshop on the Safety of Repetitive Transcranial Magnetic Stimulation, June 5-7, 1996. Electroencephalogr Clin Neurophysiol 1998;108:1-16.

Wassermann EM, Grafman J, Berry C, Hollnagel C, Wild K, Clark K, et al. Use and safety of a new repetitive transcranial magnetic stimulator. Electroencephalogr Clin Neurophysiol 1996;101:412-7.

Wassermannn EM, Lisanby SH. Therapeutic application of repetitive transcranial magnetic stimulation: a review. Clin Neurophysiol 2001;112:1367-77.

Weissman JD, Epstein CM, Davey KR. Magnetic brain stimulation and brain size: relevance to animal studies. Electroencephalogr Clin Neurophysiol 1992;85:215-9.

Weyh T, Wendicke K, Mentschel C, Zantow H, Siebner HR. Marked differences in the thermal characteristics of figure-of-eight shaped coils used for repetitive transcranial magnetic stimulation. Clin Neurophysiol 2005;116: 1477-86.

Wolpe PR. Treatment, enhancement, and the ethics of neurotherapeutics. Brain Cogn 2002;50:387-95.

Wolters A, Schmidt A, Schramm A, Zeller D, Naumann M, Kunesch E, et al. Timingdependent plasticity in human primary somatosensory cortex. J Physiol 2005;565:1039-52.

Xia G, Gajwani P, Muzina DJ, Kemp DE, Gao K, Ganocy SJ, et al. Treatment-emergent mania in unipolar and bipolar depression: focus on repetitive transcranial magnetic stimulation. Int J Neuropsychopharmacol 2008;11:119-30.

Yoshida T, Yoshino A, Kobayashi Y, Inoue M, Kamakura K, Nomura S. Effects of slow repetitive transcranial magnetic stimulation on heart rate variability according to power spectrum analysis. J Neurol Sci 2001;184:77-80.

Zangen A, Roth Y, Voller B, Hallett M. Transcranial magnetic stimulation of deep brain regions: evidence for efficacy of the H-coil. Clin Neurophysiol 2005;116:775-9.

Zwanzger P, Ella R, Keck ME, Rupprecht R, Padberg F. Occurrence of delusions during repetitive transcranial magnetic stimulation (rTMS) in major depression. Biol Psychiat 2002;51:602-3. 